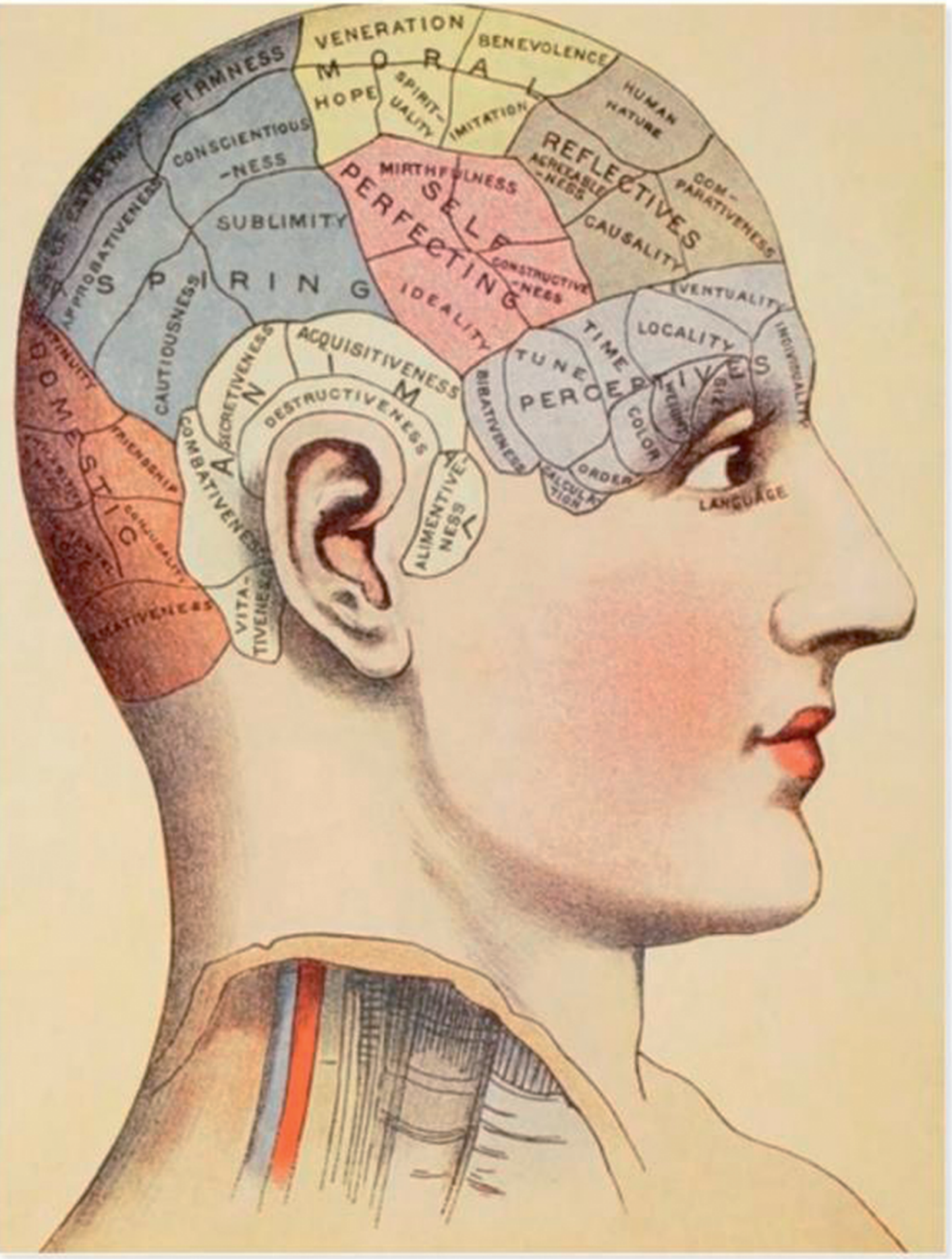

\title{
MORAL FORCES
}

Interpreting Ethical Challenges in Military Operations

Miriam de Graaff 


\section{MORAL FORCES}

Interpreting Ethical Challenges in Military Operations

Miriam Carla de Graaff 
De Graaff, Miriam Carla

Moral Forces: Ethical Challenges in Military Operations

PhD Thesis University of Twente, Enschede

Cover design: Miriam de Graaff

Printed by: Gildeprint

Lay-out: $\quad$ Nicole Nijhuis - Gildeprint

ISBN: $\quad$ 978-90-365-4279-1

DOI: $\quad 10.3990 / 1.9789036542791$

(C) 2016 Miriam Carla de Graaff, Enschede, The Netherlands. All rights reserved. No parts of this thesis may be reproduced, stored in a retrieval system or transmitted in any form or by any means without permission of the author. Alle rechten voorbehouden. Niets uit deze uitgave mag worden vermenigvuldigd, in enige vorm of op enige wijze, zonder voorafgaande schriftelijke toestemming van de auteur. 


\title{
MORAL FORCES
}

\section{INTERPRETING ETHICAL CHALLENGES IN MILITARY OPERATIONS}

\author{
PROEFSCHRIFT \\ ter verkrijging van \\ de graad van Doctor \\ aan de Universiteit Twente, \\ op gezag van de rector magnificus, \\ Prof. dr. T.T.M. Palstra, \\ volgens besluit van het College voor Promoties \\ in het openbaar te verdedigen \\ op 12 januari 2017 om 12.45 uur
}

door

Miriam Carla de Graaff

geboren op 1 september 1985

te Hengelo $(\mathrm{O})$ 
Dit proefschrift is goedgekeurd door de promotoren Prof. dr. E. Giebels en Prof. dr. D.E.M. Verweij. 


\section{PROMOTIECOMMISSIE}

\section{Promotoren}

Prof. dr. E. Giebels

Universiteit Twente

Prof. dr. D.E.M. Verweij

Nederlandse Defensie Academie / Radboud Universiteit

\section{Voorzitter}

Prof. dr. Th.A.J. Toonen

Universiteit Twente

\section{Leden}

Prof. dr. J.H. Kerstholt

Universiteit Twente / TNO

Prof. dr. kol. H.G.J.M. Vermetten

Leids Universitair Medisch Centrum

Prof. dr. G.P.M.R. Dewulf

Universiteit Twente

Prof. dr. M.T.I.B. Bollen

Nederlandse Defensie Academie

Dr. E.D. Karssing

Nyenrode Business Universiteit 



\section{Table of contents}

Chapter 1: Introduction 9

Chapter 2: Moral judgment in war and peacekeeping operations:

An empirical review

Chapter 3: On moral grounds: Moral identity and moral disengagement in relation to military deployment

Chapter 4: Emotional reactions and moral judgment: The effects of morally challenging interactions in military operations

Chapter 5: Sensemaking in military critical incidents: The impact of moral intensity

Chapter 6: General discussion

References

Summary in English

Nederlandse samenvatting

Dankwoord

Curriculum Vitae \& List of publications 



\section{Chapter 1}


"One day, I was the highest in rank at our post a few hours away from our base camp, a local man came to our gates with a terribly ill child. The man (the child's father) had overcome all his fears of retribution by the insurgents by showing up at our door. The child needed medical assistance immediately. Our medic advised that the child be transported to our base camp to obtain appropriate medical assistance. However, the base commander decided that no Dutch troops were to assist the transportation of the family. I knew that the family would probably not survive the trip due to insurgents activity in this region. [...] It was a tough call... In making the decision to obey the orders from my superiors, I had to weigh between loyalty towards my superiors, the responsibility for my men, and responsibility for the wellbeing of the local population. I decided to suggest that they go to the base camp by themselves. [...] The next morning we found the father and child killed and left behind at our gates..."

(Fragment from an interview with an army captain regarding his deployment to Afghanistan.)

Personnel in uniformed professions (e.g., police, military and fire services) are confronted regularly with ethical challenges, moral dilemmas that are inherent due to the character of their profession (cf. Richardson, Verweij, \& Winslow, 2004). In military operations, in particular, and strengthened by increasingly complex operational environments, conflicting beliefs about soldierhood, mission goals and right or wrong may become apparent (cf. Van Baarle, Bosch, Widdershoven, Verweij, \& Molewijk, 2015; Schut, De Graaff, Verweij, 2015). It is in the nature of these ethical challenges that they cannot be dealt with on the basis of regular routines or rules (cf. Thompson \& Jetly, 2014). With stakes being high, the interpretations of ethical challenges and the serviceman's subsequent reactions may have far-reaching consequences. After all, since military personnel use violence as an instrument while protecting human rights and retaining the trust of the civilian population in the area of operations at the same time, the decisions they make need to comply with social norms and the need to behave in a morally responsible way (cf. De Graaff, \& Van den Berg, 2010). If they do not, it could result (in addition to the decisions being unethical in their own right) in extreme incidents that have major operational, humanitarian or political impacts on society or the wellbeing of individuals. Especially in such high-stakes environments as military environments, the interpretation of a morally complex situation is a relevant issue to address.

For a long while, psychological and other research has studied moral judgment using a dispositional approach, in which individual features are at the center of interest. Social 
psychologists like Zimbardo - who conducted the Stanford Prison Experiment in 1971 add counterweights to the dispositional view that assumes that individual characteristics alone, such as personality traits, account for individual behavior. Zimbardo (2007) points out the relevance of situational characteristics, such as the situation's intensity that (sometimes jointly with individual characteristics) may lead to a display of acts of moral disengagement. He describes for example the 'bad apple vs. bad barrel-discussion' in relation to the Abu Ghraib abuses (Iraq) that were revealed in 2004. According to Zimbardo (2007) individuals with 'good' intentions (personality) can turn 'evil' under certain circumstances (situation), regardless of their personality: the behavior triggered will depend on the barrel that the apple is in.

How individuals deal with ethical challenges is the leading question in this dissertation, which integrates several psychological frameworks. It addresses the moral domain, focusing on issues that result from social interactions of individuals, groups, or organizations that hold an effect on the interests of other parties involved or the values of the society as a whole (cf. Haidt, 2003; Folger, Cropanzano \& Goldman, 2005). The moral domain goes beyond mere social error or personal taste and preference (e.g., celebrating Christmas in September, or eating a banana peel). It regards human life in terms of values such as justice and fairness, the protection of individual rights, and security issues regardless of personal interests of agents operating in this situation (cf. Haidt, 2003; Folger et al., 2005).

Studies within the moral domain, especially in the field of business ethics, can be divided into two realms: that of normative ethics on the one side and that of empirical (or: individual) ethics on the other (cf. O'Fallon \& Butterfield, 2005). The normative realm focuses on organizational ethics, such as behavioral codes, business ethics, legitimization of organizational activities and moral character building. On the other hand, the empirical realm addresses morality at a personal and individual level in practice and aims to explain and predict individual behavior. Studies in this realm address issues such as moral reasoning and moral disengagement.

This dissertation acknowledges the complexity of these value-laden interactions and the dilemma-charged situations that require decision-making in complex environments. Specifically, it explores the psychological mechanisms that contribute to how an individual interprets and makes sense of an ethical challenge. ${ }^{1}{ }^{1}$ will refer to this mechanism as 'moral assessment'; which I consider the first important step in the Ethical Decision Making (EDM) process required for further elaboration and action. Especially in highstakes environments, like the military, the interpretation of a situation is a relevant

\footnotetext{
${ }^{1}$ Throughout this dissertation, "we" is used instead of "I" to reflect that the studies described are a product of collaboration with my supervisors - Ellen Giebels and Desiree Verweij - and others. In the introductory and concluding chapter, however, "I" is used to highlight personal considerations and activities.
} 
issue to address. As this dissertation comprises empirical studies of individual moral assessment of servicemen in practice, it can be categorized as part of the empirical realm in moral judgment research.

\subsection{Ethical Challenges}

In this dissertation, I use the term ethical challenge, morally challenging interaction and ethical dilemma interchangeably - to refer to situations in which individuals are confronted with conflicting values and/or interests, or in which the consequences are tragic. In military operations, ethical challenges come from different sources. Van den Berg and Verweij (2006) distinguish different categories of dilemmas. The first category comprises dilemmas that occur due to conflicting positive values and obligations that are at the center of the situation. For example, when an incident occurs that should be reported but one or more of the unit members concerned is/are asked not to report it: the conflicting values in this situation are loyalty towards own unit members versus compliance with organizational procedures and standards.

A second type of dilemma results when, regardless of positive values and obligations, the consequences of possible courses of action of the alternatives may have undesirable side effects (Van den Berg \& Verweij, 2006). Under such circumstances, it may be morally responsible to not follow the obligations, and to choose the other alternative.

Within this type of ethical challenges, a specific category is distinguished, the socalled tragic dilemmas: dilemmas in which all of the available courses of action include undesirable (far-reaching negative) side effects, resulting in the belief that 'it is impossible to do the right thing' (cf. Van Den Berg \& Verweij, 2006). For example, when servicemen are confronted with armed and hostile child-soldiers who will not surrender, the dilemma arises whether or not to shoot back, which results in either injuring the children or in injuries amongst the unit members.

The premise of this dissertation is that ethical challenges are particularly likely to occur in the context of military operations because of certain organizational and contextual features of military operations that form a breeding ground for conflicting values and/or interests: (a) the high stakes, (b) the substantial cultural differences, (c) a necessity to act, and (d) a lack of an obvious solution of the situation (cf. Kramer, 2007).

Many organizations, such as healthcare institutions, install ethics committees or engage ethics consultants to deal with ethical issues (cf. Kopala \& Burkhart, 2005). Similarly, the military aims to enhance its personnel's levels of moral competence by means of ethics education and the explicit declaration of organizational norms concerning morally responsible behavior (cf. Desplaces et al., 2007; Seiler, Fischer, \& Voegtli, 2011; 
Thompson \& Jetly, 2014; Verweij, Hofhuis, \& Soeters, 2007; Williams, 2010; Wortel \& Bosch, 2011). Many of the ethics courses within the military focus on an understanding of ethical codes, dilemma training and cognitive deliberation, and reflection on challenging situations in order to explore the 'right thing to do' (e.g., Seiler et al., 2011; Verweij et al., 2007; Wortel \& Bosch, 2011; cf. De Graaff, De Vries, Van Bijlevelt \& Giebels, under review). With regard to operations abroad, however, there is often no time for extensive and time-consuming deliberation. Moreover, knowing the 'right thing to do' does not necessarily mean that individuals act upon it. For example, it took the whistleblower of the Abu Ghraib prison abuses considerable time before speaking out and intervene in the situation he considered morally reprehensible (cf. Bartone, 2007). All these challenging situations are in need of an individual understanding of 'morality'. Therefore, from low to high ranks, servicemen need to be equipped with moral competence in order to deal with these challenging situations adequately (cf. Thompson \& Jetly, 2014).

\subsection{Studies in this Dissertation - Research Aim}

Up until now, most moral judgment studies have focused on the content of dilemma situations, the outcomes of the decision-making process, and moral competence and moral development (cf. Schut, De Graaff \& Verweij, 2015; Park \& Peterson, 2006). Individual psychological mechanisms contributing to how individuals interpret and deal with authentic military ethical challenges have by and large been ignored. The main focus of this dissertation is therefore on the individual moral assessment of day-to-day ethical challenges during military missions. In order to investigate moral assessment in 'true actors', military professionals have served as research population. We also used the servicemen's personal experiences rather than theoretical or hypothetical dilemmas (such as the Trolley-dilemma and the footbridge dilemma used in other research, cf. Kahane \& Shackel, 2010). Having described the increasing complexity of today's military operations and the moral questions that are evoked in every serviceman in relation to deployments, the importance of studying the moral assessment of day-to-day ethical challenges has become evident. Therefore,

The aim of this dissertation is to expand knowledge on ethical decision-making in terms of moral assessment by examining how military professionals interpret and respond to ethically challenging situations. 
In short, the following central question forms the foundation of this dissertation:

\section{How do military professionals make moral assessments of day-to-day ethical challenges during military deployment and how does this process relate to individual responses to those ethical challenges?}

Four studies (a review study and three empirical studies) have been brought together to unravel different aspects of the moral assessment process and the influence of both individual characteristics (i.e., moral identity) and situational characteristics (i.e., moral intensity) in relation to this process is explored.

The empirical studies are largely based on two datasets. The first dataset concerns a sample of rich and in-depth interviews with military professionals from the armed forces of the Netherlands. The interviews were conducted by me and colleagues at the Ministry of Defense.

To prepare myself for the in-depth interviews and to enhance my understanding of the experiences as they occurred in their natural setting, I joined Task Force Uruzgan (TFU) at their deployment site in Afghanistan in 2010 along with a colleague who assisted me with the interviews. The data collection was launched after this preparatory phase and in-depth interviews at army camps were conducted upon return. As researchers and defense personnel, we are aware of the fact that we form part of the context of the topics addressed in these studies. Schön (1991) refers to the importance of this so-called double vision: the awareness of own attribution of meaning when other meanings can be present as well. Approaching the situation in this way opens up the possibility of using different perspectives to gain more insight. Nevertheless, we believe that we - as researchers - remained relative outsiders from the perspective of the study participants, as we were not part of their task forces and not hierarchically linked. Thus, on the one hand, we could connect to the servicemen interviewed because we spoke their language and understood the military customs. On the other hand, we were at enough distance of the participants, to be able to follow our own line of inquiry and draw our own independent inferences.

The second data set proceeds from a pilot study. From this pilot study, narratives of Dutch servicemen were administered in order to elicit prototypical dilemma situations. Two prototypical dilemma situations were derived and used in a follow-up survey study including both qualitative and quantitative research methods. Recent methodological developments argue that qualitative and quantitative methods are not exclusive and a dichotomy but part of a continuum in which integrated methodology is pursued (cf. Morgan, 2007). According to this point of view, qualitative and quantitative methods are considered equally necessary in order to obtain a meaningful and comprehensive 
image of the phenomenon under study (cf. Morgan, 2007). Using a combination of methods made it possible to explore different facets of the moral assessment process in a large sample of Dutch military personnel.

The remainder of this introductory chapter will provide a theoretical rationale underlying the central research aim of this dissertation: increasing our understanding of moral assessment in day-to-day ethical challenges. It first discusses the background of ethical decision-making and moral-judgment research. I will also define the concepts used and describe the frameworks relevant to the studies conducted.

I will conclude this chapter with an overview of the remaining chapters.

\subsection{Moral Judgment Research}

Theorists and scientists have been trying to understand morality in human life for a long time and from many perspectives (cf. Saxena \& Babu, 2013). In ancient cultures, myths and legends were used as a source for standards and norms. From ancient philosophers like Plato and Aristotle to more recent philosophers like Peter Singer, philosophers have long since addressed moral functioning. Starting early in the twentieth century, moral functioning has had the interest of psychologists and sociologists who have subsequently used a variety of perspectives to study and understand the depths of individual and collective moral behavior (e.g., Hughes, 1903; Tufts, 1909).

After the Second World War, morality received even more attention with researchers and practitioners trying to understand and explain how it was possible that 'ordinary' people behaved as cruelly as they had done during the period of war in Europe and Asia (e.g., Kohlberg, 1969; Milgram, 1963; Treviño, 1986; Haslam \& Reicher, 2007).

The research field that empirically addresses morality is that of moral-judgment research. The predominant question in this field is how individuals deal with ethical challenges, and how moral judgment works. Moral judgment refers to the processes (both conscious and unconscious, deliberate and unintentional) that assist an individual in recognizing and assessing, and eventually acting upon, a morally challenging situation (cf. Kahneman \& Sunstein, 2005).

In this section, I will provide a brief introduction to the main perspectives used in moral judgment research.

\subsubsection{Cognition vs. Intuition}

In the psychological discipline, the domain of moral judgment was initially dominated by the classical Piagetian-Kohlbergian paradigm of cognitive moral development (cf. Saxena \& Babu, 2013). Broad stages of moral cognition were proposed in the 
psychology of human development, focusing on the reasoning process of individuals when confronted with a moral dilemma (Kohlberg, 1969). Thus, in this tradition, the decision maker is regarded a rationalistic agent. A large variety of scales, such as moral reasoning (e.g., Rest; Kohlberg) and moral identity (Aquino \& Reed, 2002), have been developed and used (and been subject to critique) to measure aspects that relate to how individuals behave. This is referred to as the cognitive or rationalist approach.

From the 1970s on, a debate regarding the influence of intuitions and proneness of certain foundations in the cognitive reasoning process ensued (e.g., Gilligan, 1982; Haidt, 2001; Nucci \& Turiel, 1978). For example, Gilligan (1982) suggested that the proneness of women towards moral perspectives (or foundations) is different from that of men, resulting in the development of care ethics as opposed to the ethics of justice. This affective approach stresses the importance of emotions and intuitions as a main cause of moral judgment. Following this line of reasoning, moral judgment is caused by effortless affective intuitions, and conscious cognitions are considered as post hoc justifications of decisions made (cf. Haidt, 2001).

Recent developments have tended to combine and integrate the two perspectives. This so-called integrative approach is based on the assumption (following insights of, e.g., Sonenshein, 2007) that, in case of a disruption of the 'expected state of the world', or when there is no obvious way to engage the world, it is not a matter of deciding what to do, but a matter of interpretation. In this approach affectual intuitionism emerges, which considers ethical intuitions as cognitive moral emotions with a cognitive foundation (cf. Green \& Haidt, 2002; Roeser, 2010). This means, for example, that cognitions regarding humaneness or equality experienced in a specific situation form a foundation for emotions such as sympathy or anger. In this approach, emotions are necessary for making practical, rational decisions; emotions serve as a normative guide in moral judgments (Roeser, 2010).

A framework that offers a solution to the interpretation of challenging situations such as ethical challenges is that of sensemaking. Sensemaking is the retrospective activity an individual engages in to develop plausible explanations of what is happening in the current situation, to then be able to resume interrupted activity and stay in action (Weick et al., 2005). In order to make sense of the situation they encounter, individuals engage in certain sensemaking strategies and tactics that emerge from both an emotional as well as a rational foundation (e.g., Martin et al., 2011).

\subsubsection{Situational vs. Individual Characteristics}

The ongoing debate about the foundations of moral judgment resulted in the establishment of two influential frameworks; Rest's four components model (Rest, 1986) and Treviño's person-situation interactionist model (Treviño, 1986). 
Rest (1986) proposed a fundamental model for moral judgment with four elements:

a) moral recognition;

b) moral evaluation or judgment;

c) moral intention, and;

d) moral behavior.

Where Rest (1986) focused on individual characteristics influencing moral judgment, Treviño (1986) pointed to the relevance of situational characteristics in addition to individual characteristics. This includes, for example, the moral intensity of the specific situation, role-related responsibility (locus of control and accountability), and situational pressures such as (obedience to) authority and referent others (organizational culture). Treviño argued that although response to a moral dilemma is based on an individual's moral development, moral development alone is not sufficient to explain or predict ethical decision-making behavior (Treviño, 1986). Indeed, recent research suggests that issue-related factors like the moral intensity of the dilemma are strong predictors of the outcome of the ethical decision-making process, and sometimes even more so than personal and organizational factors (e.g., Paolillo and Vitell, 2002).

\subsection{Research in the Military Domain}

The issue of morality has been quite prominent in studies within the military domain. Research within the military mainly focused on organizational ethical issues such as policy, education and behavioral codes, however (e.g., Richardson, Verweij \& Winslow, 2004; Seiler, Fischer \& Voegtli, 2011; Tripodi, 2006; Wortel \& Bosch, 2011), or on incidents of irresponsible behavior (Bartone, 2010; Cunha, Rego \& Clegg, 2010). For example, in 'the Lucifer effect', Philip Zimbardo describes various psychological and group-dynamic processes and situational features that contributed to the escalation of the Abu Ghraib prison abuses (Iraq). Much less attention has been devoted to the role of intra-individual processes in moral judgment (e.g., Nilson, Sjöberg, Kallenberg \& Larson, 2011).

In this dissertation, a review study identifies the themes that until now have not been subject to empirical investigation with respect to moral assessment in the military. The three empirical studies that follow focus on these themes specifically, and address intraindividual processes in terms of moral assessment.

Using Rest's model as a framework, the issue of moral assessment is considered to be part of the early interpretations of the situation, resulting from moral recognition leading to moral evaluation (stage one and two). That being the case, elements from both cognitive stances as well as affective stances have their place in these studies. Moreover, 
in line with Treviño (1986), the influence of situational characteristics is addressed as well. Thus, these studies contribute to the integrative perspective.

Moral competence refers to the ability and willingness of individuals to meticulously cope with ethical challenges, i.e., "the ability to direct one's behavior towards goals that are considered worthy and good in their own right" (Park \& Peterson, 2006, p. 892; cf. Thompson \& Jetly, 2014). Within the armed forces of the Netherlands moral competence is embraced following six elements proposed by Karssing (2001) and further developed by Verweij (2005) into moral professionalism. Moral professionalism covers

(1) awareness of one's own personal values and the values of others;

(2) the ability to identify the moral dimensions in situations;

(3) the ability to come to a meticulous judgment;

(4) the ability to communicate this judgment;

(5) the willingness and ability to act accordingly; and

(6) the willingness and ability to be held accountable and feel responsible for one's personal actions in the situation (cf. De Graaff \& Van den Berg, 2010; Verweij, 2005; Wortel \& Bosch, 2011).

As stated above, I will refer to this process with the term moral competence in this dissertation because that term is common in ethical-decision making (EDM) research.

\subsection{Overview of Chapters and Additional Notes on Ethical Decision- Making}

Taking the complexity of today's military operations and previous research in the field of ethical decision-making together, this dissertation sets out to expand knowledge of moral assessment by examining how military professionals interpret and respond to ethical challenges. In order to address this research aim, four sub-questions have been formulated, more specifically defining the focus of each study this dissertation consists of. In each empirical study, different variables that came forward as issues of interest from the review study are addressed. The review study is presented in Chapter 2 .

Sub-questions:

1. What aspects of moral judgment, and moral assessment in particular, have been studied previously in a military context, and what avenues for further exploration follow this systemic review?

2. How do military personnel construct their moral identity in relation to the ethical challenges of military operations, and how does this relate to verbalized moral disengagement? 
3. What different types of ethical challenges are military personnel confronted with on a day-to-day basis during deployment, and how do these types relate to perceived emotions and the response strategies moral disengagement, numbing and relativism?

4. How is the moral intensity of an ethical challenge related to the likelihood of employment of certain sensemaking tactics in military professionals during military operations?

In chapter 2, I present a systematic overview of how moral judgment research within the military has developed in recent years. The aim of this review is to give insight into elements of moral judgment that until now have remained underrepresented in EDM research and into methodological issues in this field that are worth investigating. Together, these issues form the foundation for the empirical studies presented in this dissertation.

EDM research has shown the importance of individual features such as moral character (e.g., Chritcher, Inbar, Pizarro, 2012), moral accountability (e.g., Steinbauer, Renn, Taylor, \& Njoroge, 2014) and moral development (e.g., Verweij, Hofhuis, Soeters, 2007) in dealing with ethical challenges. These individual characteristics combine to form an individual's moral identity. The empirical part of this dissertation starts with investigating how moral assessment relates to the individual features of moral identity (Chapter 3).

Previous research has also shown the relevance of situational characteristics in moral judgment (e.g., Paolillo \& Vitell, 2002). Schut et al. (2015) point for example to the importance of the cultural context of the dilemma situation in relation with moral emotions. This dissertation therefore explores the relationship between perceived emotions and verbally expressed notions of negative assessment, i.e., moral disengagement, in different types of challenging situations during deployment (Chapter 4). Next, specific situational characteristics such as seriousness of harm, proximity and size of effect have been shown to influence moral judgment (Jones, 1991; May \& Pauli, 2002).

These situational characteristics together form the situation's moral intensity. This dissertation investigates how moral intensity relates to individual sensemaking in dilemma situations (Chapter 5). Chapter 6, finally, provides a general discussion of the studies included in this dissertation.

There are three notes I would like to make at this point. First, the chapters in this dissertation are based on individual manuscripts that are under review or have already been published. These chapters can be read separately or as a collection of explorations concerning the psychological mechanisms underlying moral assessment in EDM. As 
the chapters were written as independent articles, submitted (and some published) in international (peer-reviewed) books and journals, some overlap might occur (for example, regarding descriptions on data-collection procedures). Some inconsistencies in terminology between the chapters can also be explained by this fact (for example, the terms ethical challenge, moral dilemma and morally challenging situation are used interchangeably).

A second note concerns my employment by the Ministry of Defense of the Netherlands. Although these studies have been conducted with the consent with the Netherlands military, the statements and views expressed in this dissertation are personal and do not necessarily reflect the official organizational view regarding the matter.

A third note concerns the use of the terms ethical and moral. The term "ethical" (as is the case for "moral") often has a positive connotation, meaning that individuals display fairness and altruism (cf. De Hoogh \& Den Hartog, 2008). For example, ethical leadership is often portrayed as leaders' engagement in virtuous acts that benefit others (cf. De Hoogh \& Den Hartog, 2008). In this dissertation, however, I take a different stance in line with relevant contemporary literature (cf. Van Baarle et al., 2015): instead of focusing on outcomes of the decision-making process such as fairness and altruism (e.g., in the distribution of resources), I reserve the term "ethical" to refer to a certain state in an individual's morality. Moral judgment does not therefore refer to a process resulting solely in altruistic or fair decisions; it refers to a process in which an individual displays a certain sense of moral awareness and moral competence. The focus in this dissertation is on the first stages of the EDM process - moral assessment - and not necessarily on the outcome of the moral judgment process in terms of achieving a morally sound judgment or not. Therefore, the terms "ethical" and "moral" used throughout this dissertation do not necessarily include a positive connotation in terms of a socially valued outcome. Throughout this dissertation, in each chapter, various examples of ethical challenges are presented in order to illustrate the morally challenging situations that military personnel are confronted with during deployment.

\subsubsection{Thesis Outline}

Chapter 2 provides a systematic review of empirical research on moral judgment conducted in a military context over the past three decades. The studies are categorized in line with the aforementioned three perspectives on moral judgment (i.e., the cognitive, affective and integrative approach). This chapter gives insight in: 1) the conceptual issues -such as the studied topics distributed in these three categories, and 2) methodological issues regarding moral judgment research - such as research design and instrumental issues, population and research context. 
Chapter 3 examines the narratives of 45 Dutch servicemen in terms of the construction of their moral identity when experiencing ethical challenges in military operations. For this study, Blasi's self-model of moral functioning is used as a starting point (Blasi, 1983). Moreover, the relationship between moral identity and verbalized moral disengagement is explored.

Chapter 4 uses the same 45 narratives as a starting point and first identifies different types of ethically challenging interactions that military personnel experience during military operations. Following the somewhat overlooked affective approach we furthermore focus on the relationship between different types of ethical challenges and moral emotions and response strategies (such as moral justification, relativism and numbing).

Chapter 5 explores the relationship between the situation's characteristics in terms of its moral intensity and the use of different sensemaking tactics. Based on a pilot study, prototypical ethical challenges that are low or high in moral intensity were first selected and then presented in a questionnaire to 325 active-duty military personnel.

Chapter 6 This chapter presents the main findings of how individual servicemen make a moral assessment of day-to-day ethical challenges during military operations and the related individual responses. This chapter presents a general conclusion of the dissertation. It comprises an integrated summary of the main findings described in Chapters 2-5 that together provide an answer to the central question formulated in section 1.2. Furthermore, in this chapter, I reflect on the limitations of this dissertation and suggest avenues for future research. I conclude this dissertation in this chapter with a general discussion of the implications for theory and practice regarding moral assessment when taking these three empirical studies together into account. 


\section{Chapter 2}

\section{Moral judgment in war and peacekeeping operations:}

An empirical review

This chapter is based on:

De Graaff, M. C., F.D.A. Den Besten, E. Giebels, D.E.M. Verweij (2016a). Moral judgement in war and peacekeeping operations: An empirical review. In T.R. Elßner, \& R. Janke (Eds.), Didactics of Military Ethics: From Theory to Practice (pp. 75-11). Leiden: Brill.

The authors would like to thank Sarena Duff and Derek Suchard for their comments on previous drafts of this chapter. 
The mission of the Netherlands Armed Forces in Afghanistan included training the Afghan police and renovating their police stations by installing some basic safety measures. One of these measures included installing a munitions locker in the police station so that firearms, mines and explosives found in the police district could be stored securely. Imagine a situation in which Dutch personnel are carrying out a regular patrol mission, making contact with the local population and checking in on several of these police stations. Upon arrival at one of the police stations, the soldiers discover a woman who had been arrested earlier that day locked up in the munitions locker. The Afghan policemen inform the Dutch soldiers that, according to Afghan principles, the woman's honor is compromised if she is in a room with men other than her husband or other male relatives. So, while waiting for her husband to arrive, her honor is protected by allowing her to wait in the munitions locker. For the Dutch personnel on site, this situation creates a dilemma: should safety concerns or sensitivity to cultural norms prevail?

(Fragment from an interview with a Dutch Sergeant regarding his deployment experiences.)

This real-life example was described by a Dutch non-commissioned officer (NCO) in an interview session discussing dilemma situations during deployment. The NCO's narrative reflects the day-to-day challenges of servicemen during military operations. The scientific field that addresses such dilemmas in work contexts is that of business ethics, leaving ethics to a variety of scientists such as psychologists, sociologists, theologists and philosophers addressing ethical issues in work-contexts from their own perspectives. The domain of business ethics is commonly divided into two realms: that of normative ethics on the one side and that of empirical ethics on the other (cf. O'Fallon \& Butterfield, 2005). The normative realm focuses on organizational ethics, whereas the empirical realm addresses morality at a personal and individual level (also see the distinction made by Paine, 1994; 1996). Within organizations and societies, organizational ethics largely result in behavioral codes that guide individual actions. This field also addresses conceptual and contextual issues regarding putting ethics into practice. Research in this area deals with issues such as moral character building and training programs (e.g., Baker, 2012; Offstein \& Dufresne, 2007; Seiler \& Fischer, 2011; Wortel \& Bosch, 2011) and business ethos (e.g., Frankel, 1989; Tripodi, 2006) and considers the legitimacy of the use of force (Reichberg \& Syse, 2002). Only a few scholarly papers addressed the effects of these training programs and behavioral codes on the soldiers' moral judgment. Studies that address such 'transfer' have their place in the empirical realm (e.g., Warner et al., 2011). Research in the empirical realm - regarding individual personal morality 
- addresses individual behavior by studying and explaining events in the past and by predicting individual behavior in the future (O'Fallon \& Butterfield, 2005). Research in this area focuses on issues such as individual ethical decision-making behavior (e.g., Bagozzi, Sekerka, Hill \& Sguera, 2013; Blais \& Thompson, 2013; Simmons \& Rycraft, 2010), moral reasoning (e.g., Linn, 1989), and moral disengagement or abusive behavior (e.g., Aquino, Reed, Thau \& Freeman, 2007; Bandura, 1999; Bandura, Barbaranelli, Caprara \& Pastorelly, 1996; Hannah, et al., 2013).

Thus far, research within the military has focused largely on organizational ethical issues such as policy, education and behavioral codes (e.g., Bartone, 2010; Richardson, Verweij \& Winslow, 2004; Seiler, Fischer \& Voegtli, 2011; Tripodi, 2006; Wortel \& Bosch, 2011). Much less attention has been devoted to the role of intra-individual processes in moral judgment (e.g., Nilson, Sjöberg, Kallenberg \& Larson, 2011). This is unfortunate, because soldiers are regularly confronted with conflicting values such as in the dilemma described in the opening example of this article. Although the military has traditionally dealt with problem solving on the basis of rules and hierarchy, morally challenging situations are difficult to deal with on the basis of rules, codes and principles due to their indeterminate nature (Kramer, 2007). We define morally challenging situations as situations in which an individual is confronted with an intrapersonal 'clash' of values caused by interaction with others. Olsthoorn, Meijer and Verweij (2010) argue that current military operations tend to focus on humanitarian goals, such as winning over the local population and establishing a stable environment. These specific goals restrict the use of force to a minimum, but can cause a dilemma-rich situation. 'Civilian casualties are usually considered an unintended side effect of legitimate attacks on military goals' (Olsthoorn et al., 2010; p.141). Another dilemma is created by societies' contemporary viewpoint that military losses are unacceptable. In current military operations, military and civilian losses are unavoidable due to the risks present in the mission area. The question arises as to whether the commanders should focus on the safety of their personnel, or on achieving the military goals (Olsthoorn et al. 2010). Those (societal) restrictions and the familiarity with their comrades is most likely the reason that soldiers value the lives of their comrades above those of the local population, even though this runs counter to the basic premise of most modern military interventions (Olsthoorn et al., 2010). In military operations, the individual serviceman's competence in moral judgment is addressed, and this should be considered vital for today's military operations (cf. Richardson et al., 2004). It therefore seems important to gain insight into the intra-individual processes surrounding moral judgment in a military context. The current study aims to provide a systematic overview of empirical studies conducted on moral judgment, focusing on the specific context of the military and is a starting point for further empirical exploration. Before discussing the methodology and outcomes of this search, we first define moral 
judgment conceptually and introduce three broad frameworks for how moral judgment can be approached.

\subsection{Theoretical Background}

Moral judgment has been defined in various ways by different authors. Occasionally, the term moral judgment is used interchangeably with the terms ethical decision-making or moral reasoning (e.g., Detert \& Treviño, 2008; O'Kane, Fawcett \& Blackburn, 1996). Some define moral judgment as the ability to recognize and assess the values, norms and interests that are at stake in a given situation (e.g., Verweij, Hofhuis \& Soeters, 2007). Others define moral judgment as the evaluation of the rightness or wrongness of either the actions or the character of an individual (e.g., Haidt, 2001). As such, the individual is judged on the basis of a set of virtues held to be essential within a culture or subculture (e.g., Haidt, 2001; Pizarro, 2000). In this article, moral judgment refers to all intra-individual processes that contribute to the assessment of the cognitive, affective and behavioral reactions towards morally challenging situations (cf. Kahneman $\&$ Sunstein, 2005). This definition is the most comprehensive, since it includes both conscious and deliberate reasoning (e.g., information processing) as well as unconscious and unintentional processes (e.g., intuition and emotions). This is important to note, because moral judgment research is often outlined in fragments, focusing on a specific element in the judgment process such as cognitive processes. The fragmented elements in moral judgment research can be categorized in three main approaches: 1) the cognitive approach (also referred to in literature as the rationalist approach $\mathrm{cf}$. Sonenshein, 2007), 2) the affective approach, or 3) the integrative approach, combining cognitive and affective elements into automatic or effortless sense-making processes (cf. Greene \& Haidt, 2002; Kahneman \& Sunstein, 2005; Sonenshein, 2007).

The cognitive approach claims that individuals use deliberate and extensive moral reasoning in order to respond to morally challenging situations. This approach revolves around an individual's cognitive sophistication. Within the cognitive approach, three streams stand out. The first explains individual responses to morally challenging situations by using normative theories from traditional philosophical ethics, meaning the individual's considerations are classified as explanations drawn from the major schools of philosophy: utilitarianism, deontology and virtue ethics (Sonenshein, 2007). The second stream proposes that it is not the philosophical theory that underpins the responses in moral reasoning, but the (1) individual's personal cognitive abilities (cf. Kohlberg's (1981) stages of moral development), (2) individual difference moderators (such as moral identity, ego-strength, locus of control), and (3) situational moderators 
(such as organizational culture) (Sonenshein, 2007). The third stream of the cognitive approach is based on the issue-contingency model, which calls attention to the specific characteristics (often referred to as moral intensity) of the ethical issue itself (e.g., Morris \& McDonald, 1995; Paolillo \& Vitel, 2002).

Not all psychologists have accepted the premise that emotions should be banned from the field of moral judgment (cf. Greene \& Haidt, 2002; Thiel, Bagdasarov, Harkrider, Johnson \& Mumford, 2012). For example, Sigmund Freud (1856 - 1939) exploited emotions and intuition in his construction of the human psyche. According to Freud, the human psyche includes unconscious instincts and desires (Id), incorporation of societal values in personal moral judgment (Super-Ego), and a mediator between Id and Super-Ego (Ego). Intuition (e.g., Haidt, 2003) and emotions (e.g., Gross \& Levenson, 1993; Hutcherson \& Gross, 2011) are quite prominent in today's moral psychological research on decision-making. This approach is referred to as the affective approach. Researchers in this work field believe that many of the decisions made in daily life are rapid, effortless and unconscious (e.g., Greene \& Haidt, 2002; Haidt, 2003). In contrast to (complex) deliberation, emotions are considered to be an intuitive and biologically based reaction that influences an individual's response to important events (Gross \& Levenson, 1993). These feelings are referred to as affect-laden intuitions (cf. Greene \& Haidt, 2002) or moral emotions (cf. Haidt, 2003; Hutcherson \& Gross, 2011). Moral emotions are distinguished from general basic human emotions in terms of the "thirdparty' aspect they enclose: they suddenly appear and immediately have an affective valence regarding good or bad in interpersonal interactions (Greene \& Haidt, 2002).

When studying the decision-making process, using only the cognitive approach, the problem that individuals all make a unique construction of the situation at hand comes to the fore. Even though a situation is objectively the same, the solutions are different. On the other hand, when using the affective approach alone, only the emotional/intuitive reactions are addressed, although research shows that certain emotions promote cognitive operations such as information processing (Lerner \& Keltner, 2001; Thiel et al., 2012). The debate concerning whether to use a cognitive or an affective approach seems to resolve itself in uniting the two perspectives into an integrative approach (Lerner \& Keltner, 2001; Thiel et al., 2012). This approach is based on the assumption that - in case of a disruption of the 'expected state of the world', or when there is no obvious way to engage the world - it is not a matter of deciding what to do, but a matter of interpretation (Sonenshein, 2007; Weick, Sutcliffe \& Obstfeld, 2005). A framework that offers a solution to the interpretation of challenging situations such as moral dilemmas is that of sensemaking. Sensemaking is the retrospective activity an individual engages in in order to develop plausible explanations of what is happening in the current situation, so that the individual will be able to resume interrupted activity and stay in 
action (Weick et al., 2005). This is related to the perspective that is referred to as affectual intuitionism, which considers ethical intuitions as cognitive moral emotions (cf. Green \& Haidt, 2002; Roeser, 2010). According to this theoretical context, emotions originate from a cognitive foundation. For example, cognitions regarding humaneness or equality experienced in a specific situation form a foundation for emotions such as sympathy or anger. In this perspective, emotions are necessary for making practical and rational decisions: emotions present a normative guide in moral judgments (Roeser, 2010).

In this review, we place an emphasis on revealing trends in moral judgment research in the military by using these approaches as a manner for categorization. Previous systematic reviews on moral judgment in other or more general contexts did not primarily focus on the different conceptual approaches in this field (e.g., O'Fallon \& Butterfield). As such, this review complements existing insights in this field. Table 1 presents the operationalization of moral judgment according these categories.

Table 1 Operationalization of moral judgment in research

\begin{tabular}{lcll}
\multicolumn{2}{c}{ Framework for Moral Judgment } & \multicolumn{1}{c}{ Operationalization } \\
\cline { 1 - 2 } Approach & \multicolumn{1}{c}{ Stream } & \\
$\begin{array}{lll}\text { Cognitive } \\
\text { approach }\end{array}$ & $\begin{array}{c}\text { Normative ethics \& } \\
\text { philosophy } \\
\text { Individual's personal } \\
\text { cognitive abilities } \\
\text { Individual difference } \\
\text { moderators }\end{array}$ & $\begin{array}{l}\text { Studies addressing for example: values; virtues or } \\
\text { utilitarianism }\end{array}$ & $\begin{array}{l}\text { Studies addressing for example: moral development; moral } \\
\text { identity; moral reasoning; leadership and training effects; } \\
\text { organizational culture; coping strategies (disobedience) }\end{array}$ \\
& $\begin{array}{c}\text { Situational moderators } \\
\text { Issue contingency } \\
\text { model }\end{array}$ & Studies addressing for example: moral intensity appraisal \\
$\begin{array}{l}\text { Affective } \\
\text { Integrative }\end{array}$ & - & $\begin{array}{l}\text { Studies addressing for example: moral emotions; moral } \\
\text { stress/distress; intuition } \\
\text { approach }\end{array}$ & $\begin{array}{l}\text { Studies addressing for example: sensemaking/ } \\
\text { interpretation; experience based intuitions; integration of } \\
\text { emotions and cognitions }\end{array}$ \\
\hline
\end{tabular}

\subsection{Methods}

\subsubsection{Search Strategy}

For the purpose of this review, we applied a sensitive search strategy in order to identify relevant studies regarding moral judgment in the military context. We searched three electronic databases: Scopus, Web of Science and PsycINFO for relevant articles 
published between January 1985 and December 2013. The decision for using this time period was based on a broader review study by O'Fallon and Butterfield (2005) into ethical decision-making. They signaled a significant increase in empirical studies starting in the mid-nineties following the development of theoretical ethical models in the 1980s (e.g., Jones, 1991; Rest, 1986; Treviño, 1986; cf. O'Fallon and Butterfield, 2005). To ensure that all relevant studies regarding moral judgment in the military would be included in this review, we chose the time period from 1985 onwards.

We used the following search strategy: ('moral judgment' OR 'ethical decision making' OR 'moral dilemma' OR 'ethics' OR 'morality') AND ('military'). Limits were: 'peer reviewed journal', 'publication not older than 1985' 'language: English'. We also examined the reference lists of the identified publications. Our search strategy generated in total 820 publications in Scopus, 362 in Web of Science and 469 in PsycINFO.

\subsubsection{Selection of Studies}

The articles identified by the search were critically assessed based on the following inclusion criteria: first, the publication had to describe the empirical measurement of (elements of) moral judgment. Thus, non-empirical publications were excluded. Second, the publication's measurement of moral judgment had to be conducted in a military sample. Using these restrictions, the first author evaluated the titles and abstracts of the publications, which limited our search further to 72 publications. Thereafter, the first two authors evaluated the full content of the articles for inclusion in this review, leaving 33 publications for further in-depth analysis. Reasons for not including the remaining publications were that they did not contain any empirical data was collected or the sample used was not military. The first author reviewed all 33 studies for further analysis, while a second reviewer selected $50 \%$ of the publications at random and appraised them independently. There were no disagreements on whether to include or exclude any of these 33 publications. 


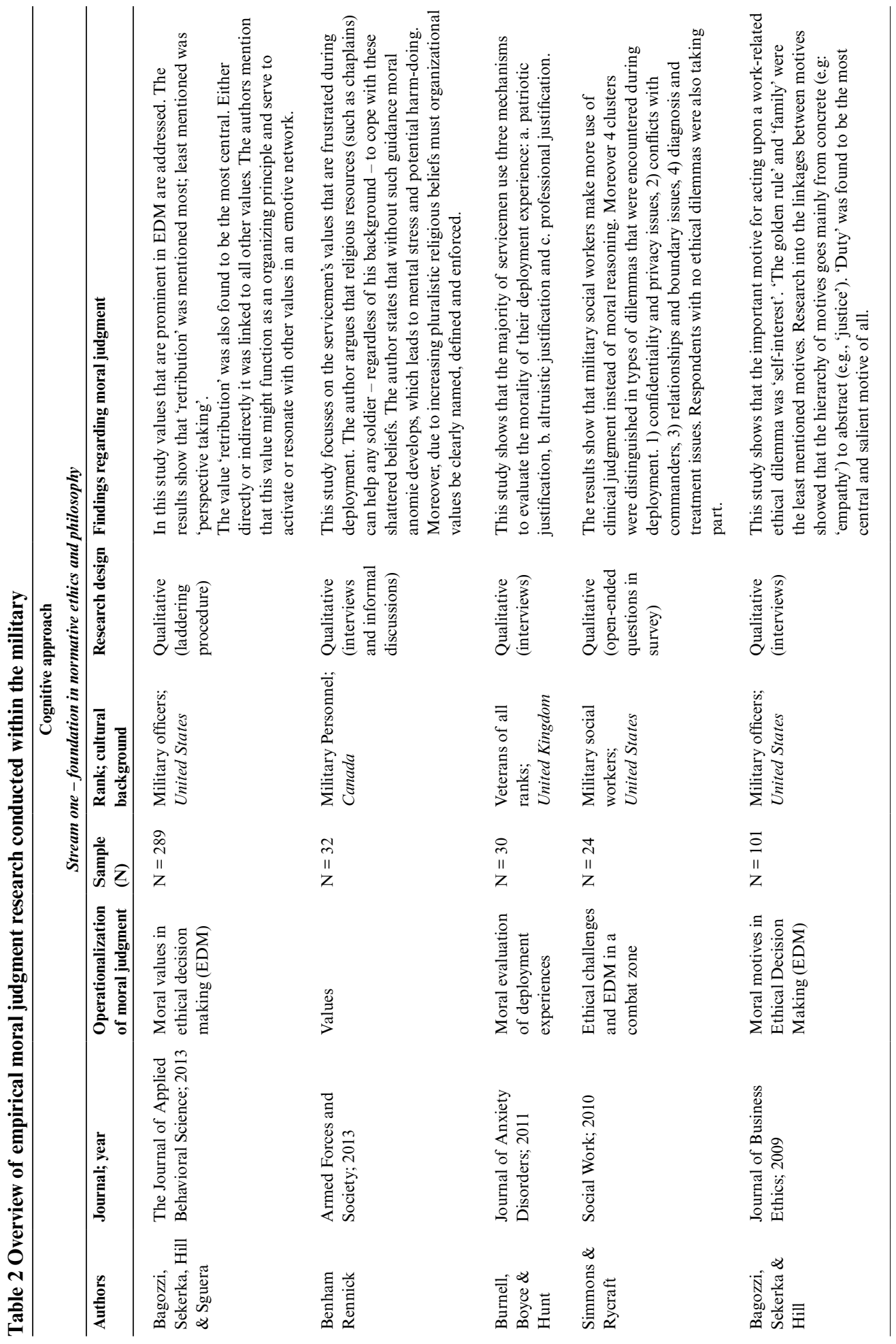




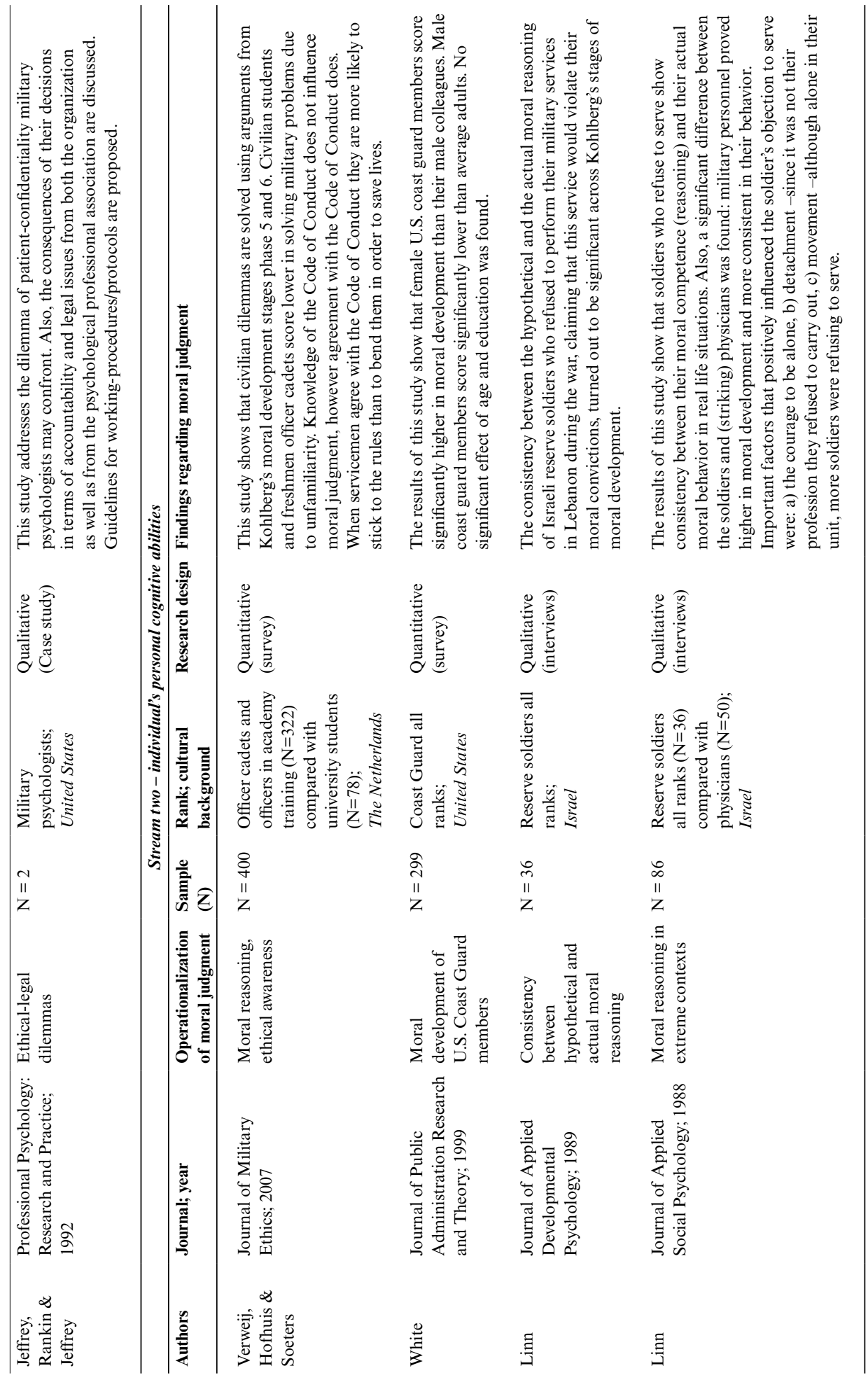




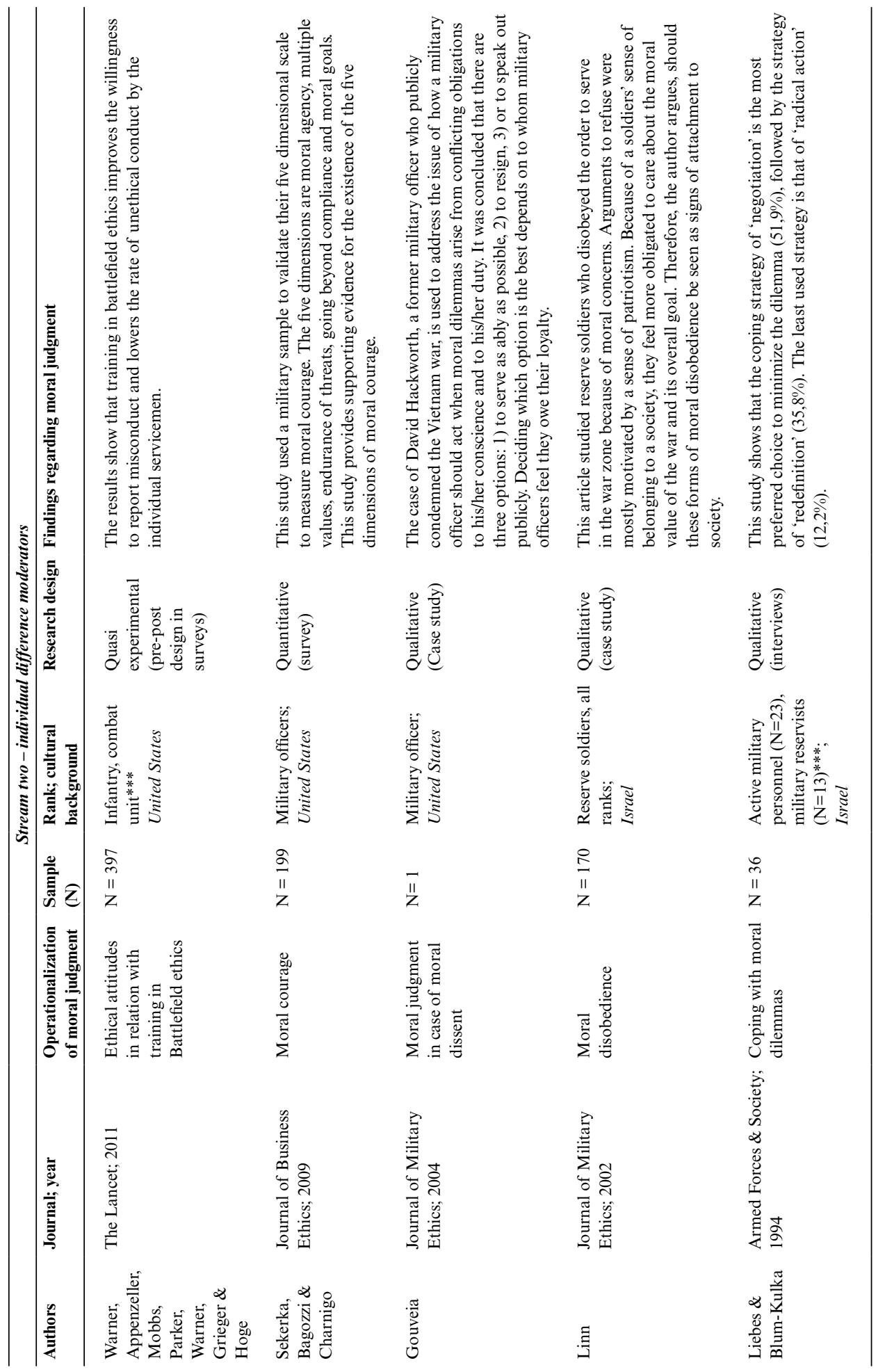




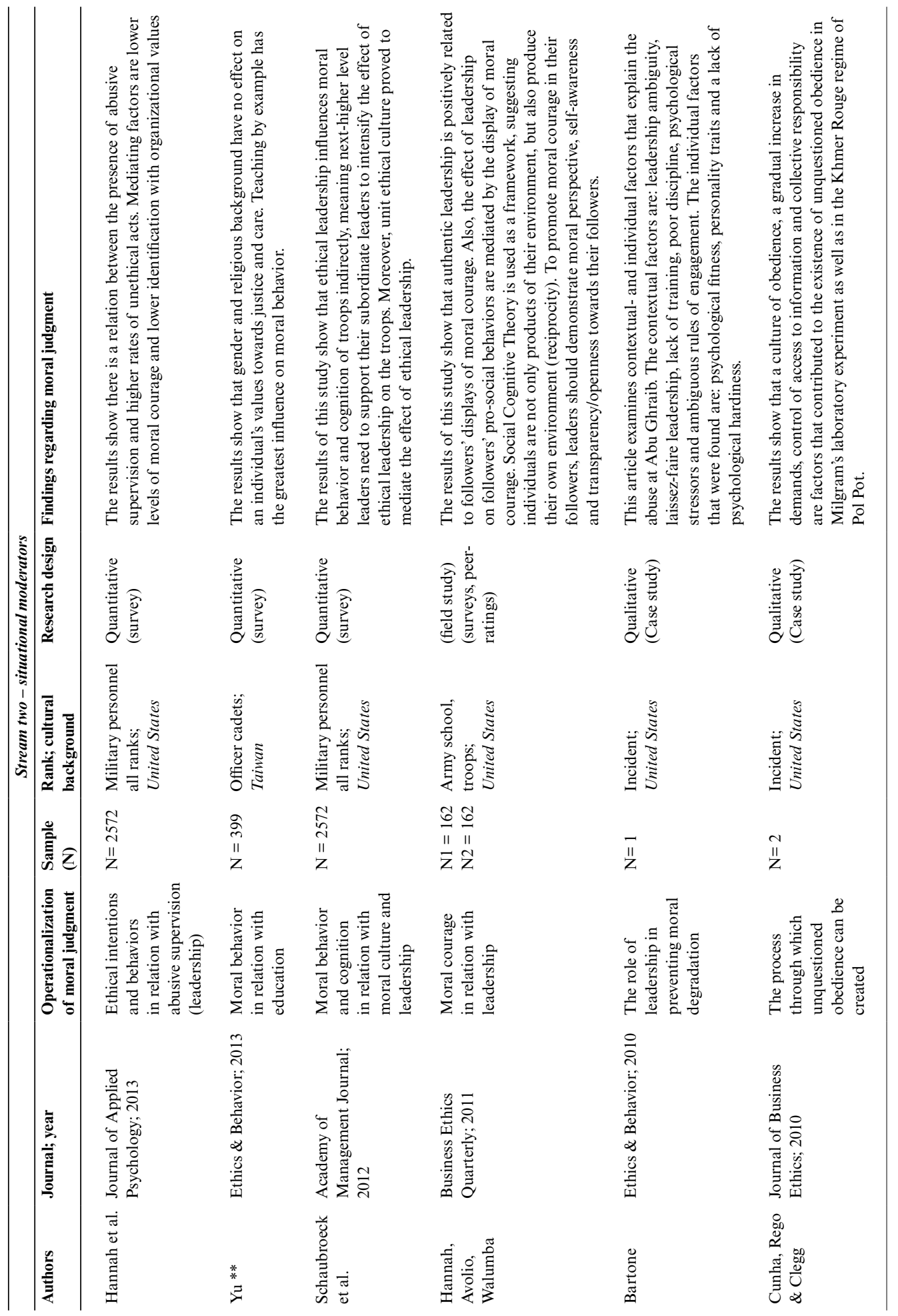




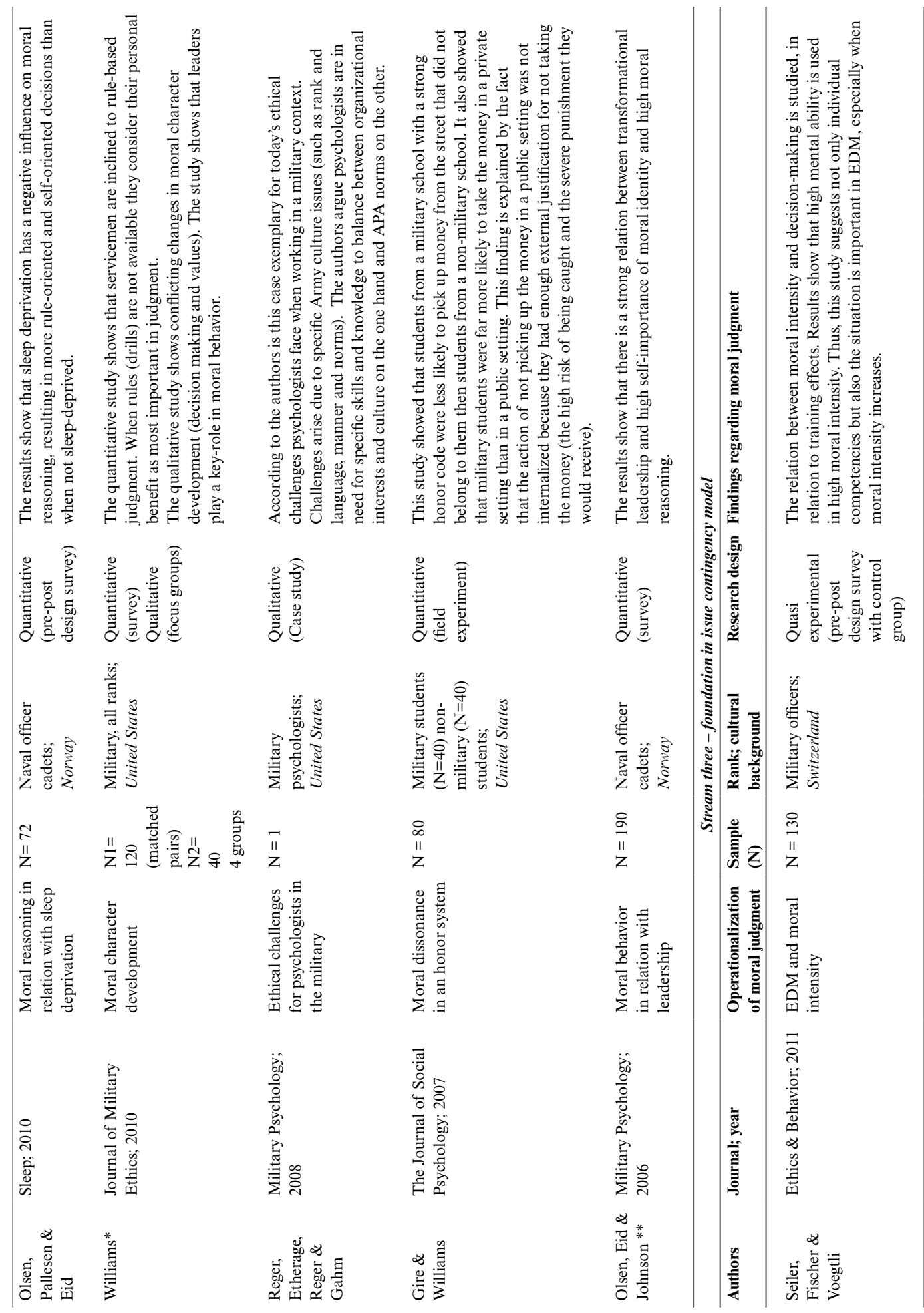




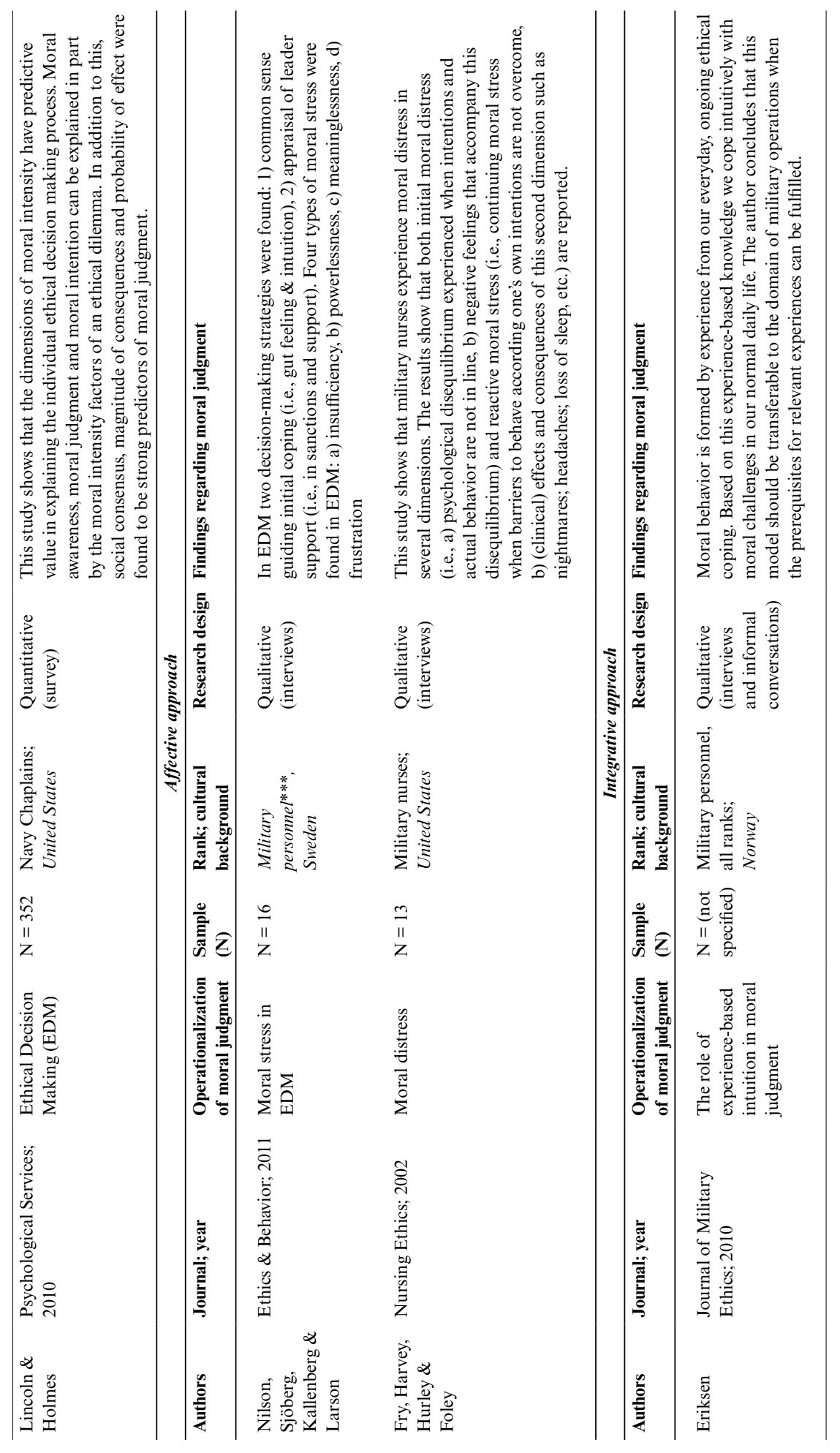




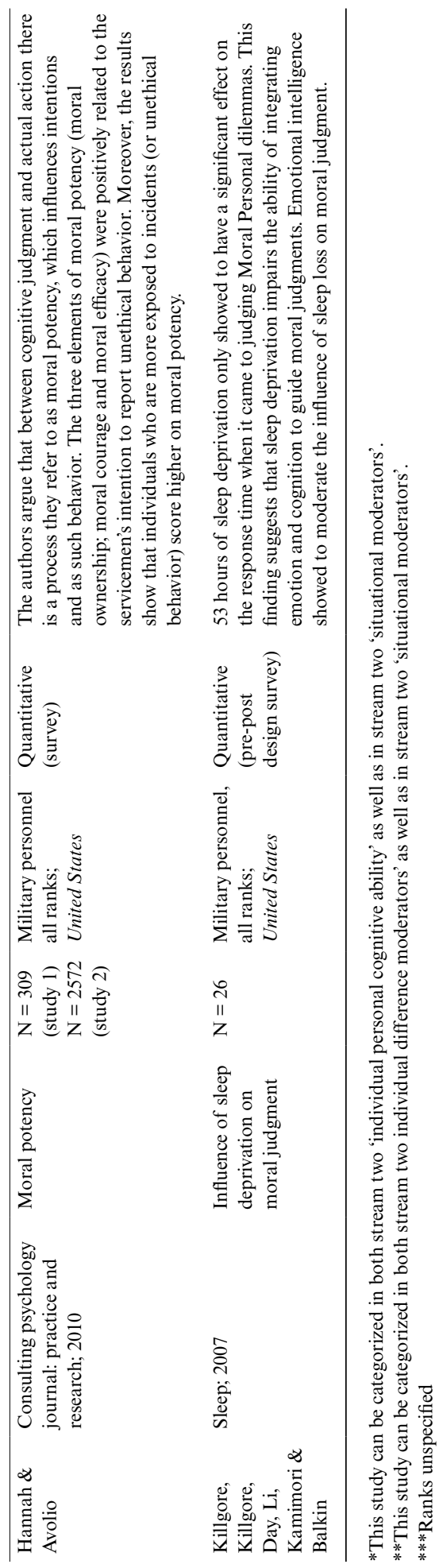




\subsection{Results}

Thirty-three references were classified as relevant with respect to moral judgment in a military context. Table 2 provides a summary of these studies in terms of the conceptual framework chosen, the methodology and the main findings of the study.

\subsubsection{Conceptual Issues}

Of the three approaches, the majority of the studies $(\mathrm{N}=28)$ used the cognitive approach to address moral judgment. Within this subsample, six studies placed the respondents' reasoning central when they were asked to describe the type of moral dilemmas they encountered (e.g., Simmons \& Rycraft, 2010) or the values that were at stake (Bagozzi et al., 2013). These studies correspond with the first stream in the cognitive approach, focusing on traditional schools of philosophy $(\mathrm{N}=6)$. These studies show, for example, that social workers encounter specific moral dilemmas during deployment due to conflicting clinical and military values (Jeffrey et al., 1992; Simmons \& Rycraft, 2010). Other studies focused on personal values that are challenged during deployment (Bagozzi et al., 2013; Benham Rennick, 2013) and addressed the organization's responsibility in order to guide and assist individual servicemen in their dilemmas (Benham Rennick, 2013). In addition, mechanisms for reflection upon and evaluating personal deployment experiences showed that a sense of 'duty' (e.g., patriotic or professional duty) is most central and salient as a motive for servicemen (Bagozzi et al., 2009; Burnell et al., 2011).

The second stream within the cognitive approach contains three subcategories. Four studies were labelled in the first category since they focused on individual's personal cognitive abilities, mostly by testing (Kohlberg's) stages of moral development (Linn, 1989; Linn, 1988; Verweij et al., 2007; White, 1999) ( $N=4)$. The higher the serviceman's moral development, the more consistent the serviceman is in actual moral behavior (Linn, 1988; 1989). Nevertheless, some scholars claim that testing an individual's morality with such scales is arguable when it comes to predicting future behavior by means of the scores (e.g., Verweij et al., 2007). Four studies fitted the second subcategory and addressed individual difference moderators such as moral identity and moral courage (e.g., Gouveia, 2004; Sekerka, Bagozzi \& Charnigo, 2009; N =4). These studies show that ethical training improves the willingness to report misconduct (Warner et al., 2011) and that moral disobedience and moral dissent are influenced by a sense of loyalty towards society (Gouveia, 2004; Linn, 2002). Furthermore, servicemen tend to cope with moral dilemmas by using rational or cognitive strategies (Liebes \& Blum-Kulka, 1994). Eight studies focused on situational moderators such as organizational culture (e.g., Reger, Etherage, Reger \& Gahm, 2008) and leadership (e.g., Bartone, 2010; Hannah et al., 2013) and were labelled as the third subcategory $(\mathrm{N}=8)$. Two of these studies focused 
on both situational as well as individual difference moderators (Olsen, Eid \& Johnsen, 2006; Yu, 2013), and another study combined situational moderators with individual's personal cognitive abilities (Williams, 2010). These studies suggest leadership (i.e., role models and examples) and hierarchy have a great influence on individual moral behavior (Bartone, 2010; Hannah et al., 2011; 2013; Olsen et al., 2006; Reger et al., 2008; Schaubroeck, 2012; Williams, 2010; Yu, 2013). For example, Hannah et al. (2013) showed that there is a relationship between the presence of abusive supervision and higher rates of unethical acts towards the local population.

The third stream of the cognitive approach focuses on moral intensity; it considers the characteristics of the situation as direct influencers of moral judgment. Only two studies addressed this issue (Lincoln \& Holmes, 2010; Seiler et al., 2011). Seiler et al. (2011) indicate that high moral ability is used in high moral intensity. Therefore, they argue, it is not only individual competencies, but also the context that is important in ethical decision-making (Seiler et al., 2011). Lincoln and Holmes (2010) highlighted that moral intensity is an important moderator in moral judgment as well. Furthermore, the magnitude of the consequences and social consensus are considered key features in this process (Lincoln \& Holmes, 2010).

Two studies used the affective approach (Fry, Harvey, Hurley \& Foley, 2002; Nilson et al., 2011). Nilson et al. (2011)'s study focused on feelings of stress and frustration in ethical decision-making and found four instigators of moral stress: insufficiency, powerlessness, meaninglessness and frustration (Nilson et al. 2011). Fry et al.'s study also focused on feelings of stress, showing there are several dimensions to be distinguished in relation with military moral challenges (i.e., initial moral distress and reactive moral stress).

Three studies used the integrative approach (Eriksen, 2010; Hannah \& Avolio, 2010; Killgore, et al., 2007). Moral potency (i.e., moral ownership, moral courage and moral efficacy) is shown to increase after exposure to incidents; it influences moral behavior by means of intentions (Hannah \& Avolio, 2010), while experience-based intuitions translate moral judgment into intuitive coping (Erikson, 2010). Furthermore, results show that sleep-deprivation impairs the ability to integrate emotion and cognition in moral judgment, causing an increase in response time in solving moral dilemmas (Killgore et al., 2007).

\subsubsection{Methodological Issues}

Research design and instrumental issues. Both qualitative $(\mathrm{N}=16)$ and quantitative $(\mathrm{N}=17)$ research methods were used in the studies of moral judgment. Of the qualitative studies, six were conducted as case studies and focused on a single event or incident (e.g., Bartone, 2010; Gouveia, 2004). The majority of qualitative studies $(\mathrm{N}=8)$ used 
interviews to examine moral judgment. The majority of the quantitative studies $(\mathrm{N}=$ 12) used surveys (self-reports). Five studies used an experimental design, such as peerratings (Hannah et al., 2011) or a pre-post test design $(\mathrm{N}=4)$. In these pre-post test design studies, respondents were asked to fill out surveys before and after training in ethical decision-making (Seiler et al., 2011; Warner, et al., 2011). Other pre-post test design studies tested moral judgment before and after sleep deprivation (Killgore et al., 2007; Olsen, et al., 2010). In one study, qualitative and quantitative research methods were combined, using a survey and focus groups for matched-pairs (Williams, 2010). This study showed that servicemen are inclined to rule-based judgment, but when no rules are available, their decisions are based on personal benefit (Williams, 2010).

Population. See table 2 for a complete overview of the number of studies conducted in different countries around the world and the professional background of the respondents. Table 3 shows that the samples of the studies were diverse: a first subsample $(\mathrm{N}=9)$ consisted entirely of military leaders, i.e., officers, or officer-cadets. Others focused solely on military personnel with specific tasks in social, psychological or medical combat support, i.e., military psychologists/military social workers/military chaplains $(\mathrm{N}=5)$, military reserve personnel $(\mathrm{N}=3)$, troops $(\mathrm{N}=1)$, or veterans $(\mathrm{N}=1)$. In eight studies, all ranks participated (i.e., officers, as well as NCOs and troops). In six studies, the ranks and professional background of the participants were not specified. Most of the studies were conducted within the U.S. armed forces $(\mathrm{N}=20)$ or within European armed forces $(\mathrm{N}=7)$.

\subsubsection{Contextual Issues}

Fifteen studies addressed moral judgment in relation to military deployment or combat situations $(\mathrm{N}=15)$. The remaining studies $(\mathrm{N}=18)$ addressed moral judgment processes in non-combat situations, such as in educational or training settings. The case studies in particular addressed moral judgment in the 'battlefield theatre', because they concentrated on incidents (e.g., Abu Ghraib prison abuse; see Bartone, 2010). Moreover, some studies asked the servicemen to reflect upon and evaluate their deployment experiences (e.g., Burnell et al., 2011). Two studies were conducted during military deployment (Hannah et al., 2013; Hannah et al., 2010). These studies focused on the display of moral courage and the occurrence of unethical acts during military operations and deployment. 
Table 3 Background of the respondents in studies regarding moral judgment conducted around the world

\begin{tabular}{|c|c|c|c|c|c|c|c|c|c|}
\hline $\begin{array}{l}\text { Cultural } \\
\text { background }\end{array}$ & $\begin{array}{l}\text { United } \\
\text { States }\end{array}$ & Canada & $\begin{array}{c}\text { United } \\
\text { Kingdom }\end{array}$ & $\begin{array}{c}\text { The } \\
\text { Netherlands }\end{array}$ & Norway & Switzerland & Sweden & Israel & Taiwan \\
\hline \multicolumn{10}{|l|}{$\begin{array}{l}\text { Professional } \\
\text { Background }\end{array}$} \\
\hline $\begin{array}{l}\text { Military officers / } \\
\text { officer cadets }\end{array}$ & 4 & & & 1 & 2 & 1 & 1 & & 1 \\
\hline $\begin{array}{l}\text { Military social } \\
\text { workers / nurses } \\
\text { / psychologists / } \\
\text { chaplains }\end{array}$ & 5 & & & & & & & & \\
\hline Reserve soldiers & & & & & & & & $3^{*}$ & \\
\hline Troops & 3 & & & & & & & & \\
\hline Infantry & $1^{* *}$ & & & & & & & & \\
\hline Coast Guard & $1^{* *}$ & & & & & & & & \\
\hline $\begin{array}{l}\text { Military } \\
\text { personnel } \\
\text { background } \\
\text { unspecified }\end{array}$ & $3 * * *$ & 1 & & & & & & 1 & \\
\hline All ranks & 3 & & & & 1 & & & & \\
\hline Veterans & & & 1 & & & & & & \\
\hline Total & 20 & 1 & 1 & 1 & 3 & 1 & 1 & 4 & 1 \\
\hline
\end{tabular}

* $\quad$ in one of these studies both unspecified active servicemen as well as reserve soldiers participated.

** in these studies all ranks of the specific Arms of Service participated.

*** of these studies, two used a case-study design.

\subsection{Discussion}

Despite the fact that ethics have received growing attention in military handbooks, training and practice, there seems to be a paucity of empirically based research in the military regarding servicemen's moral judgment. This first systematic review revealed that a total of 31 papers between 1985 and 2013 addressed intra-individual processes concerning moral judgment in the military. A factor that might contribute to this limited number of relevant papers is that research into this population is usually considered difficult. The military is an in-group oriented society (cf. Soeters, 2004) and it is difficult 
for researchers to gain access to the military population or to get them to speak freely about themselves and their actions, also because of a feeling that civilians would not understand their experiences anyway. In addition, military personnel may be reluctant to share their experiences with outsiders, not just because they are outsiders, but also because they are taught not to share information with others. Therefore, it is possible there are more, maybe classified, studies conducted.

With respect to peer-reviewed papers, our search reveals that, by far, most studies departed from the cognitive approach. This is understandable; historically, that approach has been used most frequently since the 1950s (cf. Greene \& Haidt, 2002). In line with developments within the broader psychological literature, which only recently began to address the link between emotions and moral judgment, both the affective approach and the integrative approach are less highlighted. An increase in empirical research in this field can be discerned (cf. Haidt, 2003; Rozin et al., 1999) since the 1990s. Although these trends are in line with developments in the broader field, it is also well acknowledged that the military is an organization that usually pays much attention to risk analyses, facts and checklists (De Graaff \& Van Gils, 2012). Problems are tackled using "[...] true and fixed structure[s] that can be revealed by analysis" (Kramer, 2007, p. 19). Therefore, emotions and intuitions traditionally receive less attention. At the same time, we observe a welcome increase in attention for emotion in the broader military research literature (e.g., Andres \& Rietveld, 2012; Engelhard, Olatunji \& De Jong, 2011; Weibull, 2012). This is also important because of the high levels of stress as well as the intensity of the operational context in which servicemen operate during deployment, arguably evoking strong emotions (cf. Litz, et al., 1997). A first direction for future research is therefore to further intensify the attention for the role of emotions in moral judgment notions. Due to the masculine organizational culture in military organizations, however, - in which emotions are considered unprofessional by many - it may be challenging to study emotions among active military personnel. Therefore, we suggest providing room for emotions in operational tasks (such as in debriefing-procedures) and in training programs for all servicemen, to ensure that emotions not remain unspoken.

A second observation is that the majority of the studies address moral judgment processes in non-combat settings. It can be argued that moral judgment is most crucial in the theatre of war, however (cf. Richardson et al., 2004; Seiler et al., 2011). Granted, some studies address moral judgment in the 'war zone' by means of the servicemen's personal reflection and evaluation of their deployment experiences (e.g., Burnell et al., 2011). Others focus on specific incidents (e.g., Abu Ghraib prison abuse; see Bartone, 2010). A limitation of these studies is that they are susceptible to hindsight bias and framing (cf. Podsakoff \& Organ, 1986), since the events have taken place in the past. Due to high emotional, political and legal impact these incidents often have the question 
rises whether these studies can give reliable insights in the servicemen's moral judgment in daily life during deployment. Furthermore, most studies used self-reports as a research methodology (i.e., by means of surveys). In self-reports, researchers rely on the respondents' willingness and ability to communicate about their feelings, thoughts etc. (cf. Schwarz, 1999). However, self-reports have certain limitations (see Podsakoff \& Organ (1986), for an overview). Specifically, research suggests that the use of selfreports and commonly used Likert scales for assessing responses in relation to ethical issues may be problematic for a number of reasons (cf. Janhunen, 2012). This relates to the way these items are usually framed, with social desirability issues, and with the impact of social presence, suggesting that people are more lenient in judging their own behavior, thoughts and wrongdoings than those of others (Janhunen, 2012). Also, the question arises of whether 'conventional' methods, such as self-reports (i.e., surveys), are appropriate when the conceptual framework shifts from the cognitive approach towards the affective or integrative approach. This leaves room for the exploration of authentic daily ethical challenges by means of other methods, such as narrative analysis.

A third observation is related to the research populations. The studies focus on different categories of servicemen, such as leaders or social workers. Groups of respondents were rather homogenous, in both cultural and professional background. Due to differences in training, responsibility or professional objectives (such as the difference between an infantryman and a medic), there may be group-based differences in moral judgment. We suggest that research should be conducted in a diversity of branches of service (i.e., Air Force, Marine Corps, Army, Military Police and Navy) and rank (e.g., officers, noncommissioned officers and troops).

Moreover, the studies that have been conducted until now focus mainly on Western cultures (i.e., Western Europe, U.S. and Canada), with other cultures receiving less attention. Within these Western cultures, studies conducted in the U.S. tend to dominate the field. Nevertheless, extrapolating results from U.S. military personnel onto other Western countries such as France, Norway or the Netherlands, for example, would be difficult due to the cultural differences between these countries (Hofstede, 2001). We suggest that research should be conducted in a variety of cultures, including both Western and non-Western cultures, in order to gain insights that are relevant in a larger context. Cross-cultural studies would also give insights into moral judgment in relation to cultural background. After all, an individual's cultural background is of great influence in what individuals consider moral and immoral, and in forming their moral identity and moral foundations (Haidt, Graham \& Joseph, 2009).

In terms of research content, this systematic review shows that moral judgment research has addressed themes such as moral reasoning, moral courage and moral development of military personnel, and these are well documented and studied. Also, 
certain dilemma types are distinguished, particularly confidentiality vs. loyalty issues and duty vs. personal sense of justice issues. Furthermore, the collection of works shows that leadership, sleep-deprivation and ethical-decision making training are shown to influence moral judgment in individual servicemen. However, there seems to be a dearth of work focusing on how servicemen make sense of their personal moral dilemmas as well as their behavioral reactions in the mission area. Issues such as the moral intensity of the situation and individual (moral) emotions do receive attention, but to a very limited degree. Also, as far as we know, no empirical studies address day-to-day dilemma situations in military deployment and their association with moral identity or moral disengagement.

\subsection{Practical Considerations}

In addition to the scientific considerations described in the sections above, we believe it to be relevant to note some practical considerations that may be drawn from this overview regarding the studies conducted. After all, military ethics were developed to be of service for the military professionals who are supposed to carry out tasks in challenging and high-stake situations (cf. Cook \& Syse, 2010). The importance of moral judgment in the military is undisputed. Nevertheless, it is not uncommon for servicemen to question whether ethics is a field worth considering in the military arguing that it is something for the armchair philosopher, but not at all useful for the 'boots on the ground' (cf. De Graaff \& Van den Berg, 2010). This may be caused by the fact that indeed few answers are available for the servicemen with practical questions. Where organizations like financial banking institutions, universities and medical centers have ethical committees that help individuals with their doubts and dilemmas regarding their profession, such committees seem unavailable in the military. After all, different organizations are available to assist servicemen to cope with difficult situations (such as mental health departments and chaplains services). There is also a legal department available (even during military operations). Practical ethical guidance for 'small questions' is unavailable, however. The ethical committees that do exist concentrate primarily on resolution of incidents.

Many defense organizations rely on the use of Codes of Conduct and (legal) rules instead. Although servicemen tend to use rule-based judgment (Williams, 2010) the effects of having a Code of Conduct remain vague (cf. De Graaff \& Van den Berg, 2010). After all, Verweij et al. (2007) showed that agreement with the content of the Code of Conduct influences moral judgment, whereas simply knowing its content does not. Also, moral reasoning (knowing what the right thing to do might be) and actual moral behavior is not necessarily consistent under different circumstances (Gire \& Williams, 2007; Linn, 
1988). Therefore, it seems insufficient for the organization to trust that when a Code of Conduct is published all employees will respond with morally responsible behavior. Or, as Verweij (2013), states: "For it is obvious that a definition of responsibility is by no means a guarantee for actual morally responsible behavior [...]" (Verweij, 2013, p. 26). Thus, transference from the Code of Conduct to for example ethical (dilemma) training and moral character building seems advisable. Focusing solely on strengthening the moral character of the individual serviceman is not enough to ensure morally responsible behavior, however. After all, the organizational culture, group dynamical processes and leadership behaviors are shown to influence the individual's behavior as well (Cunha et al., 2010; Hannah et al., 2013; Olsen et al, 2006). Moreover, situational factors such as the intensity of the situation at hand should not be overlooked (cf. Zimbardo, 2007). In adequate moral judgment, a balance is found between the perspective of the individual, the context and the organization (cf. Verweij, 2013). Notably, teaching morally responsible behavior in an adequate way, within the military, will have to start by acknowledging that 'bad apples' may not be intrinsically bad; they may come to be because of 'bad barrels'. Zimbardo (2007) used the metaphor of 'bad apple - bad barrel' in relation with the Abu Ghraib Prison incident in Iraq in which U.S. soldiers violated prisoners' human rights and abused Iraqi prisoners. Zimbardo suggests that organizational and situational circumstances contributed to individuals behaving in this way. According to this presumption, the circumstances were crucial factors in this incident.

To conclude, in current military operations abroad, interactions with local populations and allied forces are more common than ever (Moelker, 2013). Consequently, morally challenging situations are inevitable due to new ways of operating (cf. Richardson, et al., 2004). It is our hope that this review will stimulate additional research in this discipline, as well as inspire others to conduct similar research in a wide range of high-stakes situations such as, for example, situations faced by law enforcement and humanitarian aid workers. 



\section{Chapter 3}

\section{On moral grounds: Moral identity and moral}

disengagement in relation to military deployment

This chapter is based on: De Graaff, M.C., Giebels, E., \& Verweij, D.E.M. (under review). On moral grounds: Moral identity and moral disengagement in relation to military deployment.

We would like to express our gratitude to Eva van Baarle and Derek Suchard for their comments on previous versions of this chapter. 
A UN observer meets an old lady at the gates of the camp every day. She is an outcast in the nearest village, desperately needs food, and is basically starving. UN regulations prohibit any intervention, so the UN observer is not allowed to give her any food. One day, the woman witnesses food packages being brought to the camp for the staff. She comes begging for food to save her life. The dilemma arises of whether the observer should follow the mission guidelines or give humanitarian aid.

(From an interview with a Dutch UN observer regarding his experiences during military deployment abroad in Angola.)

Difficult decisions that can sometimes have dramatic consequences are at the heart of the military profession. When interacting with others, an intrapersonal 'clash' of values (i.e., rights, standards and norms) can occur, leading to an ethical challenge (cf. Sparks \& Siemens, 2014). In this study the term ethical challenges refers to issues that result from collisions of two or more moral values that, although each is worthwhile in its own right, cannot be respected at the same time (cf. Van Baarda \& Verweij, 2006; De Graaff, De Vries, Van Bijlevelt \& Giebels, in press). These ethical challenges result from social interactions (between individuals, groups, or organizations) that affect the interests of other parties concerned or the values of society as a whole, such as justice, humaneness and fairness (cf. Haidt, 2003; Folger, Cropanzano, \& Goldman, 2005). Others have referred to such situations as moral dilemmas (e.g., Van Baarda \& Verweij, 2006). Servicemen sometimes do not frame the situation as a moral dilemma per se, however, but do recognize that a moral choice is at issue and sometimes experience it as uncomfortable and problematic; we therefore prefer the term ethical challenge.

It is in the nature of ethical challenges that they cannot be dealt with on the basis of regular procedures or rules. Their resolution requires the application of elevated levels of an individual's moral competence (Thompson \& Jetly, 2014). Moral competence refers to the ability and willingness of individuals to cope meticulously with ethical challenges, i.e., "the ability to direct one's behavior towards goals that are considered worthy and good in their own right" (Park \& Peterson, 2006, p. 892; cf. Thompson \& Jetly, 2014). Moral competence is interwoven with an individual's character or identity (Park \& Peterson, 2006). That is, an individual's level of moral competence relates to that person's level of moral development, defined as one's identity construction in moral terms (Desplaces, Melchar, Beauvais \& Bosco, 2007). In many studies addressing morality, definitions of moral and ethical include positive aspects referring to fairness and altruism. In this paper, however, such a positive connotation is not presumed. In line with the definition of moral competence, moral or ethical are adjectives used to refer to all processes involved in moral judgment that combine awareness of the moral dimensions in a situation (e.g., 
rights and values at stake), critical deliberation, and judgment processes (e.g., to consider possible solutions and consequences of actions). As such, these adjectives simply refer to the fact that the process involves an ethical matter.

Until now, empirical research into moral identity in relation to the military has been scarce, while other elements of the concept of self, such as social identity, have received much more attention (e.g., Van den Berg \& Soeters, 2009). The primary aim of this study is therefore to explore the construction of the service member's moral identity in relation to ethical challenges encountered in military operations.

Military organizations aim to enhance their personnel's levels of moral competence by means of ethics education for all ranks (cf. Desplaces et al., 2007; Seiler, Fischer \& Voegtli, 2011; Williams, 2010). Many of the military ethics courses focus on a correct understanding of ethical codes, dilemma training or cognitive deliberation and reflection upon challenging situations (e.g., Seiler et al., 2011). Often, however, there is no time for extensive and time-consuming deliberation during complex military operations. Therefore, decisions in the area of operations are often made rather intuitively and splitsecond. In these cases, such decisions - which frequently have profound consequences may then be reflected on in the aftermath of the event. It is at that moment in particular that soldiers may consider their behavior in the context of their moral framework. This may lead to feelings of distress and guilt when personal standards are challenged. In order to cope with those feelings, recent research indicates that servicemen may verbally demonstrate various mechanisms of moral disengagement (cf. De Graaff, Schut, Verweij, Vermetten \& Giebels, 2016b; Grassiani, 2012). Moral disengagement is a psychological mechanism that individuals use to dissociate themselves from what they believe is unethical conduct and to justify their own actions (Bandura, 1999). Whether this happens as a result of or in spite of ethics education is an open question. Theorists argue that for individuals with a strong and central moral identity such processes are less likely to occur. Little empirical research is available, however (Walker, 2004). The second aim of this study is therefore to gain some initial insights into the relationship between moral identity and mechanisms of moral disengagement in military deployment. After elaborating on the prevalence of ethical challenges during military deployment, we will discuss conceptual frameworks for moral identity and moral disengagement, as well as their interconnection. 


\subsection{Ethical Challenges during Military Deployment}

Contexts of military deployment are arguably more susceptible to ethical challenges than many other professional environments. After all, military operations are likely to come hand in hand with the experience of conflicting values when taking into account the different perspectives from, e.g., colleagues, the opposing military forces (OMF), the local population and the broader political and social context (Thompson \& Jetly, 2014).

The following example, drawn from our interview material shows the difficult choices military personnel need to make.

During an anti-piracy mission, a commander in the Royal Netherlands Navy faces a dilemma. His mandate prohibits giving humanitarian aid on land in the vessel's patrol area, such as medical assistance to the local population. The commander knows that his personnel can easily help the people living near the beaches, especially medically, without compromising the mission. He can order the marines on board to build an improvised platform in the water near the beach, which can easily be reached by the locals in order for them to obtain medical assistance. This way, he both complies with the mandate and follows his personal moral urge to help these people. The commander knows, however, that even though this construction is possible, it might only be for a short period of time. After all, if the situation were to change and risks increase, he may no longer be able to provide such help. Additionally, when another coalition partner takes over command in this area, the new commander may well decide to discontinue these activities. So, the Dutch commander wonders whether he would really be helping the local population by giving aid and he asks his advisor what to decide. What is the 'right' decision for him to make?

\section{(Fragment from an interview with a Dutch Naval officer.)}

As the complexity of today's military operations increases, the ambiguity regarding the dualistic nature of the soldier's roles in the mission area increases as well (Broesder, Op den Buijs, Vogelaar, and Euwema, 2014). For example, Perez (2012) describes the ethical and political obligations servicemen have in the mission area in addition to the obligations they have as military professionals in terms of their warrior-effectiveness. These obligations become even more challenging as a result of (1) the rapid technological developments in the armaments industry leading to potentially more severe consequences of one's actions, and (2) changes to the configuration of deployment units, with the rise of a general tendency to decentralize decision-making, leaving more room for the service 
members' own assessment of the situation and requiring higher-level competencies (cf. Richardson, Verweij \& Winslow, 2004).

The ethical challenges that servicemen encounter during military operations show similarities to what is known as a double bind in communication and learning research (cf. Visser, 2003). According to this theoretical perspective, a situation becomes a double bind when:

a) two or more people are involved in an intense relationship - e.g., leader and subordinate(s);

b) messages are incongruent;

c) the receiver of these incongruent messages cannot withdraw from the situation or comment on it; and,

d) the double bind tends toward self-perpetuation, meaning that when a situation becomes ambiguous and incongruent, this state tends to be long-lasting and difficult to overcome (cf. Visser, 2003).

For example, on the one hand, the servicemen are trained to act loyal towards their comrades (safety and protection) and the mission (striving for successful completion) as duties. On the other hand, and at the same time, their objective in peace operations is to bring peace, stop human rights violations and establish a stable environment. This may bring about conflicting roles of warrior (ethos) versus peacekeeper, resulting in confusion and strain regarding their own role, identity, and priorities. Because of their own personal standards, which may deviate from what is required during specific assignments or because of ambiguous mission goals, service members may struggle in the fog of war with what the acceptable or 'right' thing to do is (cf. Grassiani, 2012; Olsthoorn, Bollen, \& Beeres, 2013). The challenge in many of these operations is that no obvious best solution exists, either morally nor strategically, while - at the same time - there is a necessity to act.

\subsection{Moral Identity}

In the 1950s, Erikson introduced the concept of identity in psychology, departing from a child-development perspective (see Erikson, 1946). Since then, different theoretical perspectives have been discussed and studied (cf. Blasi \& Glodis, 1995). Despite these different perspectives and emphases, it is generally acknowledged that the essence of identity concerns the answers to the basic question: 'Who am I?' Following this line of inquiry, individuals are observers of themselves as they carry out intentional actions and go through a wide variety of situations they experience. Identity is therefore a subjective concept of the self as subject (Blasi \& Glodis, 1995). It is important to note 
that this subjective experience of the self is not only a matter of deliberate self-reflection, it is also grasped immediately, indirectly, and unintentionally at the moment of action and experience (Blasi \& Glodis, 1995). Janoff-Bulman (2004) describes how individuals struggle to make sense of the tragic and intense events they encounter. She claims that such events can shatter people's fundamental assumptions about themselves and the world in general (Janoff-Bulman, 2004). The question of 'Who am I?' might therefore very well be answered differently after having experienced high-impact events.

In order to make morally-informed decisions under challenging circumstances, the moral identity, that part of an individual's self that addresses the moral domain, needs to be activated. Moral identity consists of elements of the self that translate considerations, judgments, principles and ideals into moral schemas, which in turn motivate action (Aquino, Reed, Thau \& Freeman, 2007; Blasi \& Glodis, 1995). Research suggests that moral identity may become salient when people attach more value to this part of one's identity than to other parts (Thoma, Rest \& Davison, 1991) or when the situation requires immediate moral action, such as when one is confronted with an ethical challenge. Self-understanding is considered to be important precondition for a well-established moral identity (Reimer, DeWitt Goudelock \& Walker, 2009).

Blasi's self-model of moral functioning, is an important framework for moral identity (Walker, 2004). The model integrates moral cognition and moral personality in order to explain moral behavior (Walker). It consists of three sub-categories: 1) the moral self, 2) the individual's sense of personal responsibility, and 3) self-consistency (Blasi, 1983; Walker, 2004). The first sub-category of 'moral self' refers to the centrality of morality in considerations, including the salience of morality in one's identity. Blasi argues that morality may have differing degrees of centrality in individuals' lives: for some moral standards and values are well integrated in their daily-activities whereas for others such integration seems to be minimal (Blasi, 1983; Walker, 2004). The second sub-category, sense of personal responsibility, refers to the extent to which individuals consider themselves responsible for their own actions and the situation at hand. Highlevel feelings of responsibility can be categorized as proactive (having an intrinsic sense of responsibility) or reactive (a sense of being held accountable). The third subcategory, self-consistency, refers to the reflection upon the integration of values and moral standards in daily activities. According to Blasi self-consistency is a fundamental motive in functioning that can be satisfied by congruence between judgment and action. That is, individuals evaluate their own behavior by comparing it with their personal moral compass in order to come to a sense of self-integrity (cf. Walker, 2004). These three subcategories together form a coherent moral identity referring to a certain state of being regardless of whether the individual is considered a 'good' person. Thus, they do not describe a certain level of moral development. 


\subsection{Moral Disengagement}

Bandura (1999) suggests that moral disengagement permits an individual to selectively reframing his or her own actions in order to isolate those actions from one's personal standards of what is morally acceptable (Bandura, 1999). Blasi argues that the moral identity is strongly intertwined with these selective disengagement processes since individuals are prone to maintain their perceived self-integrity (Walker, 2004).

Bandura distinguishes three main categories of moral disengagement. The first centers around the reconstruction of own behavior or the incident itself. This may involve masking what happened by using ambiguous language, or justifying actions by comparing personal behavior to inhumane actions carried out by other parties. The second category includes the reconstruction of one's personal role in the incident, for example by arguing that one's personal role is only minimal and one cannot be hold accountable. The third category includes reconstruction of (the role of) the parties involved, like blaming the victim or dehumanization. We already suggested that the primary reason for moral disengagement is that in concrete situations during operations, servicemen are often inclined to act quickly and to reflect on the decisions they made only afterwards. Such decisions, often between two equally good values, are likely to create discomfort because choosing one, almost automatically implies violating the other. For example: one of the sergeants we interviewed described how he felt torn between his girlfriend's peace of mind and seeking her help and comfort by telling her about the horrors he experienced in Afghanistan. Normally, they would tell each other everything. He desired to talk to her since one of his close friends and fellow company man was killed in an IED strike, but he was afraid to share this with her because it could disturb her. Whatever choice the sergeant made would present him with feelings of discomfort. After all, telling her could disturb her peace of mind since she would be concerned for his well-being, not telling her would disturb him since he desired her comfort and wanted to maintain the open and sharing relationship they had. Such discomfort may give rise to self-regulation processes, presumably to ultimately maintain a positive selfimage (cf. Higgins, Bond, Klein, \& Strauman, 1986).

Regardless of the actual situation at hand, it is the subjective perception in the aftermath concerning the morality of the actions that may cause the servicemen to exhibit mechanisms of moral disengagement (Grassiani, 2012). Typical manifestations of moral disengagement include: dehumanization, disavowing responsibility and justification, euphemistic labeling (i.e., masking what happens, for example by using terms such as 'collateral damage' to describe civilian casualties), and advantageous comparison (i.e., comparing what happens with other situations that are considered worse, such as stating 
that torture is permitted as the victim has killed innocent children; Bandura, 1999; see also, Table 4). Crosby (1994) describes how an American soldier -after having shot an unarmed German soldier who was trying to surrender during WWII - sought out the chaplain when his feelings of guilt grew too strong ' $[. .$.$] and tried to justify his action$ by reasoning that "it was either him or me." (Crosby, 1994 p.135 in: Wead, 2015). In addition to the response strategies that Bandura labeled as moral disengagement, other studies have addressed the relevance of other response strategies such as relativism and numbing in order to justify one's own behavior. For example, one reason that some servicemen give for not taking action is that they do not feel competent to intervene in local issues that are culturally determined since these are impervious to change (e.g., Schut, de Graaff, \& Verweij, 2015; Whetham, 2008). In addition to moral identity, this collection of research suggests that the use of response strategies in a military context, specifically moral disengagement, calls for further study.

\subsection{Method}

In order to generate a construction of the phenomena of moral identity and moral disengagement in their social context, a Grounded Theory (GT) research method was used. In such descriptive/exploratory research, data collection and analysis are interrelated processes: researchers start with a relatively 'empty head' in order to discover 'a theory' by means of observations of social phenomena that are part of the studied reality (cf. Corbin \& Strauss, 1990). We therefore used abduction - a qualitative research strategy within GT - with the theories described in the introduction as sensitizing concepts or heuristic tools. They were used in order to reflect on the material and eventually result in directions for future research (Richardson \& Kramer, 2006). Moreover, after noting patterns, themes and ideas concerning relationships between variables in a qualitative manner, quantitative analyses were added to our basic qualitative approach. This is in line with recent methodological developments, arguing that qualitative and quantitative methods are not exclusive and a dichotomy but part of a continuum wherein integrated methodology is applied (cf. Morgan, 2007). From this perspective, qualitative and quantitative methods are seen as equals in obtaining meaning and developing a more comprehensive picture of the phenomenon, rather than one being privileged over the other (cf. Morgan, 2007). The different variables were systematically analyzed, which made it possible to explore the relationships between the variables. 


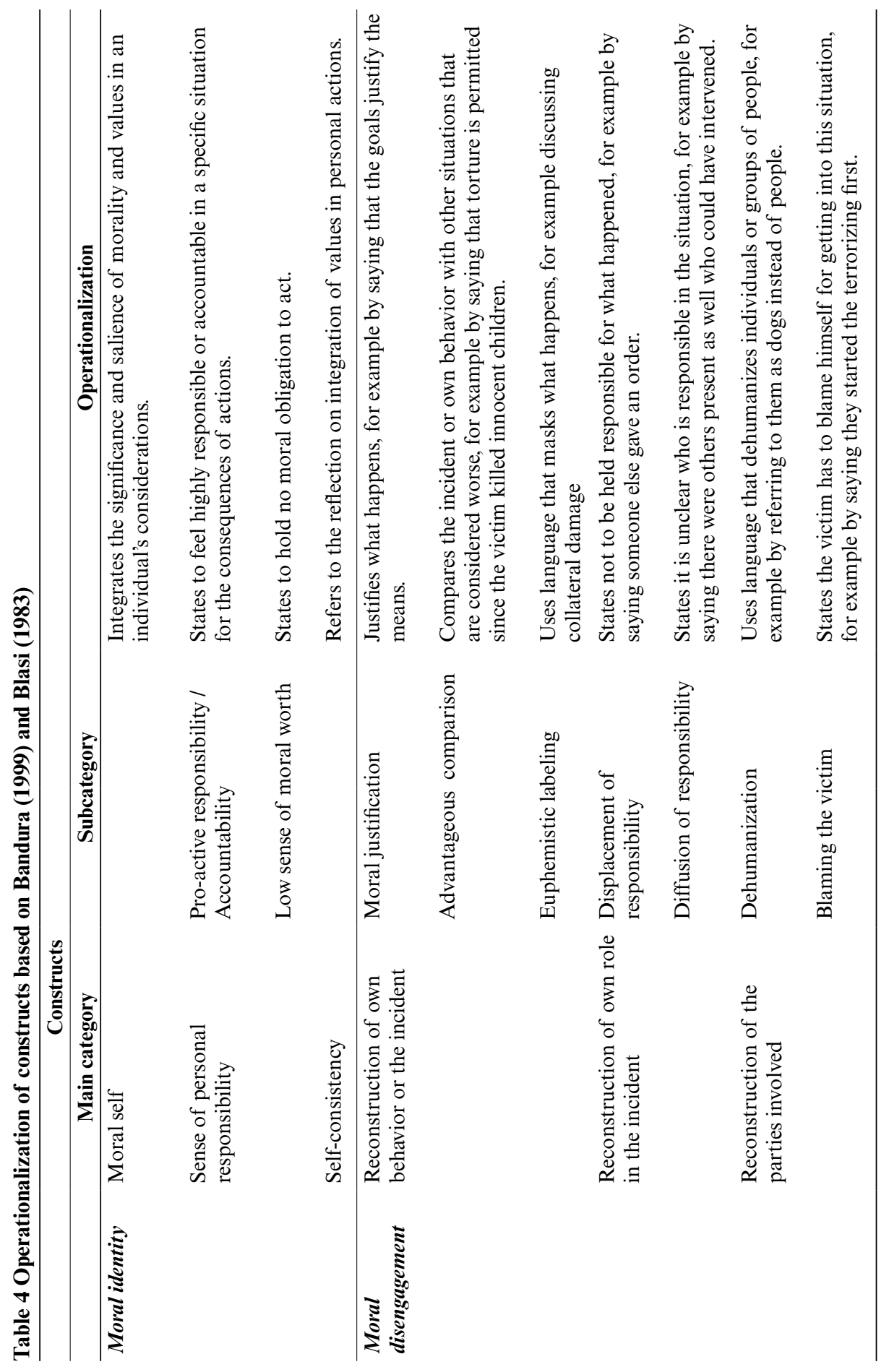


Interviews have proven to be an effective instrument and make it possible to capture a wide range of information regarding ethical issues in a military context (cf. Benham Rennick, 2012; Nilsson, Sjöberg, Kallenberg \& Larsson, 2011). The contents of the transcripts of in-depth interviews with active serving military personnel were therefore analyzed according to a GT approach (cf. Corbin \& Strauss, 1990)².

With regard to the first aim - gaining insight in the subject's moral identity during military operations - Blasi's theoretical self-model of moral functioning (Blasi, 1983) was used as a starting point for abduction. The second aim of this study was to gain insight in the relationship between moral identity and moral disengagement in military deployment. Bandura's theory on moral agency was used as a starting point with respect to this aim (Bandura, 1986; Bandura, 1999).

\subsubsection{Sample}

The brigade commander in charge of personnel deployed to Afghanistan in 2009-2010 was asked to give his consent for us to approach his units. After having received consent, personnel who had been deployed in Afghanistan under ISAF command (from 20062010) were invited to participate. We especially focused on servicemen who were part of operational units (such as combat units and provincial reconstruction teams), as those units were most likely to have experienced contact with the local population, other coalition partners, and high-risk situations. Thereafter, in accordance with snowball sampling, additional participants were recruited for participation (Noy, 2008). The criteria for inclusion were: (a) being in active military service at the time of the interview, (2) having had deployment experience abroad no longer ago than two years prior to the interview, (3) having experienced direct contact with the local population and/or coalition forces during a military deployment. In total, 60 servicemen from different branches of service were sent a personal invitation to participate on a voluntary basis in the research project. We explained that the study aimed at gaining insight into deployment experiences and their impact on the daily life of individual service members. The participants were informed that the general research results would be used for educational and scientific purposes and for pre-deployment training programs, but they were not informed about the exact research goals. Moreover, they were informed that their individual results would not lead to clinical assessments or treatments since this study is not related to mental health. All interviewees were ensured confidentiality, meaning their commanding officers or other third parties would not be notified of their individual answers.

\footnotetext{
${ }^{2}$ See for a complete overview of the interviewing techniques and methodology: De Graaff, M. C., Schut, M., Verweij, D. E.M., Vermetten, E., \& Giebels, E. (2016). Emotional Reactions and Moral Judgment: The Effects of Morally Challenging Interactions in Military Operations. Ethics \& behavior, 26(1), 14-31.
} 
A total of 45 service members voluntarily agreed to participate, and 45 meetings were arranged. A further 11 who had replied positively to the invitation were not included for a variety of reasons such as not meeting the research criteria or unavailability at the time of the research. Most of the participants had been deployed to Afghanistan. Others had been deployed for peace operations in Bosnia, Somalia, Angola and elsewhere. The participants differed in age and rank: varying from 18 years to 47 years old $(M=28$ years; $S D=8.3$ ), and from private up to lieutenant-colonel. As is the case in the general military population, the number of women participants was modest $(\mathrm{N}=7,15.5 \%$; in the general population of the Dutch forces the percentage of female servicemen averages around 10\%, Ministerie van Defensie, 2015).

\subsubsection{Instrument and Procedure}

Interview format. Data were collected by means of qualitative semi-structured interviews following a prepared interview guide, which was pre-tested among a small number of servicemen before using it in the main study. This interview guide ensured that all participants were asked similar questions by two behavioral scientists. One of the interviewers (the first author of this article) was continually present throughout all interview sessions, whereas the second interviewer position was shared between two researchers. Both interviewers were affiliated with the Netherlands Ministry of Defense. Regular informed consent procedure was followed.

A single interview session lasted approximately 50 minutes. The interviews were all digitally recorded and transcribed afterwards. During the interview sessions, openended questions covering basic demographic information (such as age, rank and professional function) were asked and the participants' experiences in the mission area were discussed. This was needed in order for the participants to construct internalized and evolving stories (narratives) in which they would address their moral identity. The participants were stimulated to share their experiences, so open-ended questions were used such as 'Can you give us an example of a situation that was difficult to cope with during deployment?' This gave us the opportunity to elaborate on the respondent's remarks. For example, we used follow-up questions addressing their moral identity with questions like: 'Can you describe what it was that made this specific situation so difficult, what was at stake?' and, 'Can you describe a situation in which your values or norms were challenged?' or, 'Can you describe how you look back on your own behavior and the behavior of others at that time?' For all prompts, participants were also asked if they could reproduce more examples up to the point where they indicated there were no more.

Operationalization of concepts. Blasi's self-model of moral functioning was used in order to code and interpret moral identity in the narratives. Bandura's framework was used as a starting point for the operationalization of cognitive reconstructions of their actions to make them acceptable, i.e., moral disengagement (Bandura, 1986). 
Coding procedure. The recordings of the interview sessions were transcribed and systematically content analyzed by three raters, using a coding guide covering all constructs (see Table 4). Markers that highlighted key aspects of the construct, were drawn from the responses of the pilot study. This coding guide was also used in previous research (see: De Graaff et al., 2016b). First, two transcripts were independently labeled in terms of moral identity remarks and moral disengagement strategies by all three raters and jointly discussed afterwards. This led to several categories and markers to be adjusted or narrowed down to more generally applicable codes. The resulting coding scheme was then used to let two coders independently code four interviews. Cohen's Kappa was relatively low (.43), which appeared to be due to one rater leaving several narratives uncoded. It was decided that the rater who had left narratives blank needed to code these specific sections again (unaware of the labels used in the other rater's codings). This was performed and led to a higher Kappa: .78, which reflects substantial interrater agreement. The two raters labeled the remaining interview transcriptions (Cohen's Kappa displayed .78 for the remaining transcriptions as well).

\subsubsection{Analyses}

After coding all narratives, we established the frequency of remarks about moral identity and moral disengagement (per sub-category). The means, standard deviations, and correlation between the variables measured in this study are presented in Table 5 .

The mean scores of a variable represent the average number of remarks made per participant for each category across the 45 interviews. In addition to the separate subcategories of moral identity, a sum score of the three elements of Blasi's model was computed (referred to as 'overall moral identity score'). On average, participants made almost 5 remarks referring to their moral identities $(M=4.76 ; S D=3.94)$. The higher this score, the more pronounced the moral identity can be regarded for an individual. With regard to moral disengagement, we decided to use a global score for moral disengagement by computing all expressions of moral disengagement per participant. On average, the participating servicemen reported 2 such acts $(M=2.27 ; S D=2.96)$.

\subsubsection{Demographic Variables}

The number of participants in the different categories was not high enough to make useful and relevant distinctions in demographic variables such as age, gender and rank. We did, however, include the question of how many deployments the participants had experienced (i.e., a first deployment versus multiple deployments) as well as their positions in the chain of command (i.e., in a leadership or in a subordinate position). 


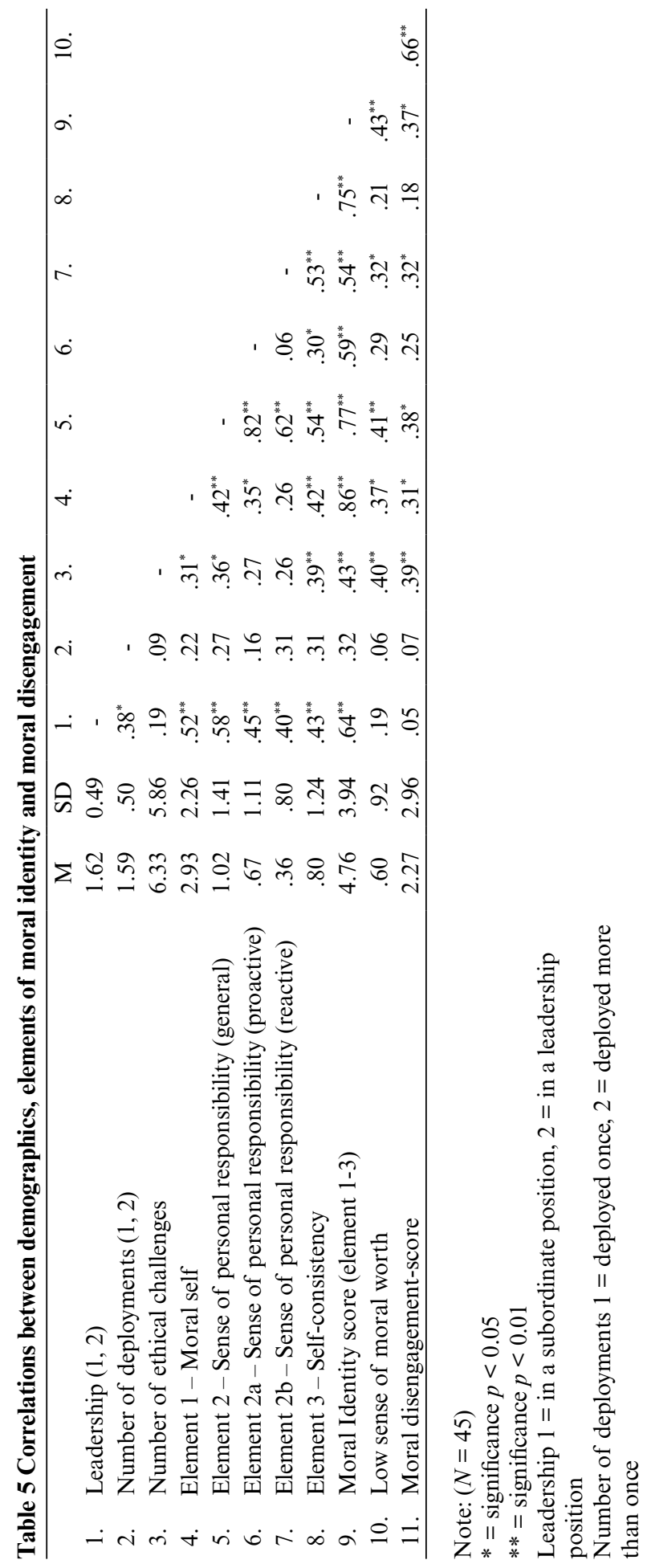


The first distinction was included because everything is experienced for the first time in a first deployment and thus may evoke more intense experiences or more elaborate reflections, while having had multiple deployments may make people more accustomed to the challenges they encounter. We considered leadership position to be a relevant variable to include because military leaders are subjected to ethics education more than their subordinates and their (sense of) responsibility may be higher due to their formal position.

Ethical challenges. Finally, we also included the total number of ethical challenges described by the participants in the analyses. In this, only newly mentioned (not repetitive) ethical challenges were taken into account.

\subsection{Results}

\subsubsection{Descriptive Data for our Main Constructs}

All narratives were coded for the operationalization of the different variable. For each narrative, the times a variable (i.e., moral identity; moral disengagement) was mentioned was determined. Also, ethical challenges described by the participants in their narratives were coded separately. The means, standard deviations, and correlations are presented in Table 5. Table 5 shows that in total, 285 separate dilemma situations were described $(M=6.33 ; S D=5.86)$. These included three types of ethical challenges:

a) (Local-)cultural-related challenges ( $M=2.55 ; S D=2.35)$, for example: $A$ female $U . N$. observer is the only woman in the camp and, due to cultural differences there are frequent discussions between her and her coalition partners - from another ethnic background - concerning the things she can and can't do. She understands that the coalition partners feel uncomfortable with her wearing sporting shorts and T-shirts when exercising. Although she outranks the coalition partners, she feels that her position as one of the few officers present is under strain. She considers whether she should conform to the norms of the coalition partners' culture regarding interactions with women and change her exercising routine although this would mean she would not be able to participate in all the sports events with her Dutch colleagues.

b) Work-related ethical challenges

i) Own troops-related $(M=4.67 ; S D=4.12)$, like: An assignment is given, but not formally ordered. The platoon commander is asked to carry out the assignment immediately. There are limited resources and limited time for preparation, however. This puts the platoon commander in the dilemma of whether to carry out the assignment, knowing that doing so puts his team in a greater risk since a complete risk analysis is not possible, and that failing to do it might have negative consequences for the mission. 
ii) Coalition force related $(M=1.73 ; S D=1.10)$, like: During deployment, the Dutch troops cooperate with allied forces. One of the allies trains in an urban area. The story goes that civilians were wounded and houses were damaged during this training. The Dutch find this unacceptable. Nevertheless, they are also ordered to carry out patrol missions together with these allies. The commander of the patrol mission of coalition forces is torn between loyalty towards the allies and empathy for the local population.

a) Personal ethical challenges $(M=1.50 ; S D=.80)$, for example: One of the sergeants described how he felt torn between his girlfriend's peace of mind and seeking out for her help and comfort by telling her about what he experienced during his deployment in Afghanistan. Normally, they would tell each other everything. He desired to talk to her since one of his close friends and member of his company was killed in an IED strike. He was afraid to speak to her about this, however, because it could disturb her, leaving her worrying about his own safety. Whatever choice the sergeant would make, it would present him with feelings of discomfort. After all, telling her could disturb her feelings since she would be concerned for his well-being, not telling her would disturb him since he desired her comfort and wanted to maintain the open and sharing relationship they had.

With regard to the moral identity of servicemen during military deployment, the participants made a total of 132 remarks that refer to what Blasi refers to as their moral self, i.e., the significance and salience of morality issues for their considerations $(M=$ 2.93; $S D=2.26$ ). For example, 'I consider humaneness as the most important value in my life'. A total of 46 remarks were made regarding the second element of Blasi's framework: sense of personal responsibility $(M=1.02 ; S D=1.41)$. Of these 46,16 remarks relate to accountability $(M=.36 ; S D=.80)$ and thirty cover a sense of proactive responsibility $(M=.67 ; S D=1.11)$. For our further analyses, the sum score for sense of personal responsibility was used. For example: 'As their commander, I am responsible for their wellbeing, so I do not like it when I don't have the time to think over a mission'. The participants also made 27 remarks indicating a low sense of moral worth $(M=.60 ; S D=.92)$. For example: 'Who am I to have an opinion about that?' Finally, 36 remarks were made regarding the third element of Blasi's self-model of moral functioning, self-consistency $(M=.80 ; S D=1.24)$. For example: 'Looking back on our actions, I wonder: am I what I have done? I have done things I would never do under normal circumstances.' Overall, we found that the three sub-categories of moral identity were positively associated (all $r>$ $.42, p<.01)$. Thus, the more participants were inclined to mention something in one category, the more they also referred to the other moral identity sub-categories (e.g., moral self is positively associated with sense of personal responsibility). 
A total of 102 remarks about moral disengagement were made. The overall moral disengagement score $(M=2.27 ; S D=2.96)$ was used for further analyses without going in to the different mechanisms specifically. Moral justification was mentioned most $(M=1.38 ; S D=2.09)$. A Dutch infantry Sergeant explained for example: 'You need to understand that we all live in different worlds. The world at home, the world at work, the world during deployment. Those are all separate worlds, with their own and differing standards about right and wrong. You consider those standards as completely 'true' in that specific world, even when they are not 'normal' back home. So that's why you do, what you do...'Advantageous comparison was not mentioned at all. Dehumanization $(M=.04$; $S D=.21)$ and diffusion of responsibility $(M=.04 ; S D=.21)$ were expressed least.

\subsubsection{Correlational Analyses}

The correlational analyses (see Table 5) show that there is a positive relationship between two elements of moral identity (i.e., moral self and sense of personal responsibility) and the moral disengagement-score. Thus, the more pronounced the moral side of one's identity, the more mechanisms of moral disengagement were uttered. Also, the number of ethical challenges mentioned and remarks about a low sense of moral worth display a positive relationship with the moral disengagement score. This indicates that individuals who experience more ethical challenges or consider themselves as being less capable to act upon a situation express more justifications for their actions (or lack thereof). Interestingly, low sense of moral worth is positively related to the sense of responsibility in general and with accountability, but not with proactive responsibility. This suggests that an intrinsic willingness to take responsibility is not related to whether or not an individual believes that (s)he has a moral obligation to act, while for responsibility in general and for accountability this does seems to be case.

\subsubsection{Deployment Experiences}

Independent sample t-tests were conducted in order to examine the relationship between deployment experience (i.e., a first deployment $(\mathrm{N}=15)$ versus multiple deployment $(\mathrm{N}=22)$ experiences $)$ and the sub-categories of moral identity and the construct moral disengagement (see Table 6). A significant difference was found for the overall moral identity score $(t(35)=-2.00, p<.10)$, which can be attributed to differences in self-consistency $(t(35)=-1.90, p<.1)$ and a specific element of sense of personal responsibility namely accountability $(t(35)=-2.27, p<.1)$, with servicemen who had multiple previous deployments mentioning these elements more often. 
Table 6 Means and SDs for main constructs in relation with deployment experience (one/two or more)

\begin{tabular}{lccccc}
\hline & \multicolumn{2}{c}{$\begin{array}{c}\text { One } \\
(\mathbf{N}=\mathbf{1 5})\end{array}$} & \multicolumn{2}{c}{$\begin{array}{c}\text { Two or more } \\
(\mathbf{N = 2 2})\end{array}$} & \\
\hline & Mean & SD & Mean & SD & $\mathrm{t}$ \\
\hline Moral identity score & 3.53 & 3.31 & 6.14 & 4.22 & $-2.00^{*}$ \\
Moral self & 2.53 & 2.30 & 3.59 & 2.44 & $-1.32, n s$ \\
Sense of personal responsibility & .60 & 1.35 & 1.36 & 1.40 & $-1.65, n s$ \\
\multicolumn{1}{c}{ Accountability } & .07 & .26 & .45 & .74 & $-2.27^{*}$ \\
Pro-active responsibility & .53 & 1.30 & .91 & 1.07 & $-.96, n s$ \\
Self-consistency & .40 & .74 & 1.18 & 1.47 & $-1.90^{*}$ \\
Moral disengagement-score & 2.13 & 3.23 & 2.59 & 3.20 & $-.43, n s$ \\
Low sense of moral worth & .67 & .90 & .77 & 1.02 & $-.33, n s$ \\
Ethical challenges & 6.00 & 5.94 & 7.14 & 6.65 & $-.53, n s$ \\
\hline
\end{tabular}

*significant difference at .1 level

Table 7 Means and SDs for main constructs in relation with leadership-position (Yes/No)

\begin{tabular}{lccccc}
\hline & \multicolumn{2}{c}{$\begin{array}{c}\text { Yes } \\
(\mathbf{N = 1 7 )}\end{array}$} & \multicolumn{4}{c}{$\begin{array}{c}\text { No } \\
(\mathbf{N = 2 8})\end{array}$} \\
\hline & Mean & SD & Mean & SD & t \\
\hline Moral identity score & 7.94 & 4.18 & 2.82 & 2.16 & $4.69^{* * *}$ \\
Moral self & 4.41 & 2.65 & 2.04 & 1.40 & $3.42^{* *}$ \\
Sense of personal responsibility & 2.06 & 1.60 & .39 & .79 & $4.01^{* * *}$ \\
$\quad$ Accountability & .76 & 1.09 & .11 & .42 & $2.38^{*}$ \\
\multicolumn{1}{c}{ Pro-active responsibility } & 1.29 & 1.45 & .29 & .60 & $2.73^{*}$ \\
Self-consistency & 1.47 & 1.63 & .39 & .69 & $2.60^{*}$ \\
Moral disengagement-score & 2.47 & 3.22 & 2.14 & 2.84 & $.36, n s$ \\
Low sense of moral worth & .82 & 1.19 & .46 & .69 & $1.29, n s$ \\
Ethical challenges & 7.76 & 6.88 & 5.46 & 5.08 & $1.29, n s$ \\
\hline
\end{tabular}

* significant difference at .05 level

** significant difference at .01 level

*** significant difference at .001 level

\subsubsection{Leaders and Subordinates}

Independent sample t-tests were conducted in order to address the relationship between being in a leadership position and expressions of ethical challenges, moral identity elements and moral identity. No significant differences between leaders $(\mathrm{N}=$ 17) and subordinates $(\mathrm{N}=28)$ were found for the number of ethical challenges reported, low sense of moral worth, and moral disengagement. For the three elements of moral identity described by Blasi, the leaders scored significantly higher than their subordinates (see Table 7). 


\subsection{Conclusion and Discussion}

To learn more about how service members deal with ethical challenges, the present study explored the construction of moral identity in military operations and its relationship to moral disengagement. Semi-structured interviews with 45 service members were content-coded and analyzed.

The results indicate three patterns. The first pattern from the correlational results suggest that a higher ethical awareness of moral challenges in the situation (i.e., a stronger moral identity and a higher awareness of ethical challenges) is associated with more justifications for one's own behavior. Thus, when moral identity is more prominent and the number of experienced ethical challenges is high, the participants express more rather than fewer mechanisms of moral disengagement. It is possible that the more one considers oneself to be a morally responsible person, the more necessary it seems to restore violations of this self-image when having to deal with double-bind issues. This is in line with earlier research that showed that individuals who act in line with their values feel better about themselves (Hitlin, 2007). A possible explanation for this outcome might lie in the nature of these 'double bind' challenges (Visser, 2003). The double bind reflects an incongruity between two (or more) self-guides; self-directive standards for being (e.g., Higgins et al., 1986). For example, in our interviews a female captain expressed how she considers the mission goals to be ambiguous: 'Our commanding officers told us our mission was to get back home in one piece, as there was too much political fuzz. I still believe that is a strange goal: why do we [the military] go to these areas just to get back safely? Then we'd better not go at all. In my opinion, we are there to establish safety and security, and that means that sometimes people get hurt. I understand that we desire to get back all in one piece, but that can hardly be the primary goal of our military operation?!'

It is likely that the servicemen with a more central moral identity will be more prone to recognize the double binds in their professional duties or the core values that are not acted upon, and will therefore experience that choosing one line of action will have possible negative consequences for the other (cf. Broesder, et al., 2014). In such situations, where actions simply cannot match all of the self-guides, feelings of discomfort easily arise (Higgins et al., 1986). Arguably, moral disengagement may leverage this negative state for example by justifying the choices made. Thus, the more one perceives ethical dilemmas to exist, the more it is necessary to alleviate the tension resulting from choosing only one value or option, but disregarding another one. This requires adequate training and preparation of military personnel in order to stimulate them to behave in a morally competent way. After all, research has shown that successful manifestations of ethical decision-making are an important reason to persist in commitment to moral competence (cf. Reimer et al., 2009). These double binds may be more present for individuals who 
hold leadership positions than for troops, as leaders hold responsibility for operational mission goals, the well-being of subordinates, and human rights of all parties involved. Interestingly, this pattern was observed regardless of rank.

The second pattern we found suggests that leaders (officers as well as NCOs) are more inclined to developed moral identities than their subordinates. Firstly, they score significantly higher on moral self, i.e., the significance and salience of morality issues for their considerations. This finding indicates that the participants are quite capable of describing values and principles that are important to them and that they are able to describe situations where their (core) values were challenged. This can be explained by the fact that they receive more extensive ethics education than their subordinates. Secondly, their sense of proactive personal responsibility is significantly higher, which arguably follows from the duties connected to their role. Thirdly, leaders score higher on self-consistency. This might be due to the fact that they are aware of the role model function they hold for many of the troops, so it is good they reflect on their actions. No significant differences between leaders and subordinates (or: non-leaders) were found in utterances of moral disengagement and low sense of moral worth, however. The fact that leaders display a similar (low) sense of moral worth as their followers is notable. After all, the leaders are who are assumed to be in a position to make a difference and to initiate change, in their own team, their own organization and in interaction with local populations and coalition partners.

Finally, when looking more closely at the number of utterances, a third pattern becomes clear. Regardless of their position as leader or subordinate, or whether they had more than one deployment, participants described approximately six ethical challenges on average. This hints at a well-established level of moral awareness. The fact that they are able to recognize difficult situations does not necessarily reflect a high level of moral competence or taking responsibility, however, since this implies more than just recognizing the challenge as such (cf. Park \& Peterson, 2006). Critical self-reflection and self-assessment (measured in self-consistency) are relatively underreported even though moral identity is quite prominent in the servicemen regardless of their rank. These results may hint at the strong urge of individuals to maintain a positive self-image (cf. Hitlin, 2007): When individuals are well aware of the ethical dimensions of the situations they find themselves in, they may also be more sensitive to their own roles in those situations. So, when their actions run counter to their personal beliefs of being a 'good person' they tend to use response strategies like moral disengagement in order to maintain their sense of 'good self'. As such, it may point at a possible drawback of fostering a strong moral identity. In line with this observation, remarks indicating self-consistency were not made very often. It appears that participants are able to describe the ethical challenge and what they believe to be at stake, but they seem to be less prone to reflect upon their actions and thoughts. 
The observation that servicemen do not reflect much on their behavior following ethical challenges even when they appear to have a strong moral identity offers a clear line of inquiry for both research and practice. After all, such insensitivity may lead to moral blindness: a 'normalization' of or inattentiveness for actions that are morally reprehensible. Such moral incapacity - i.e., not acting when individuals normally think they ought to - is also found in other studies in relation with cultural critical situations (e.g., Schut \& Moelker, 2015). In one of the interviews, a Dutch platoon commander described how he justified his failure to answer with an adequate intervention in a situation where humaneness was at stake for local children, as they were being abused by adults of their village. 'I have been wondering whether I would act similar to them if I would have grown up under these circumstances. [...] But, well, that is how it goes. That is what those people think is right. How they learned to deal with things. Realizing this meant that I could cope with such abuses of what I consider respect and humaneness. It is just what it is. So that's why I believed we couldn't intervene in cultural concerns like in this situation with these children.' An army captain described how he feels that he is becoming inattentive during his deployment to Afghanistan: 'When I first arrived in the mission area, things seemed so much worse than later on. When time passes, you just get used to how shitty things are. You don't see the wrongdoings of anyone anymore. And you don't mind the challenges you are confronted with. Seeing the first 'horrors' makes you feel sick, but after a while you don't notice it that much anymore.'

\subsubsection{Practical Implications}

Training and preparation sessions could benefit from creating awareness of and training in dealing with these ethical challenges. This might also mitigate possible negative side-effects (wrong-doings) that are 'approved' by moral disengagement. Especially since our research suggests that having a strong moral identity is not sufficient: it seems to promote rather than hinder the cognitive process of moral disengagement. It is therefore important that servicemen be educated as well in how ethical decision-making and related processes such as moral disengagement work. Recent studies investigating the relationship between moral reasoning and mindfulness indicate that intentionally paying attention in an open way enhances awareness of the experience and contributes to the moral reasoning process (e.g., Ruedy \& Schweitzer, 2010). Intentional contemplation or reflection is therefore relevant in order to make sense of the moral dimensions in a situation. When this reflexivity, or critical thinking, is indeed minimal for the servicemen, military organizations should integrate this aspect more extensively in ethics training programs, in order to stimulate meticulous decision-making. Another suggestion would be to create adequate mandates for the completion of the mission that leave room for individual consideration in order to meet the requirements of specific ethical challenges, 
instead of the contradicting and ambiguous ones the servicemen are now struggling with (cf. De Graaff \& Van Gils, 2012).

\subsubsection{Limitations and Future Research}

Even though the present study proposes interesting avenues for further research, some limitations need to be addressed. Narrative analysis has some limitations. For example, we could only address the verbal reactions of service members. Our material is therefore dependent on the verbal and reflexive capacities of the participants and their willingness to spontaneously share these thoughts and feelings. According to Haidt (2001) individuals only start to deliberate on intuitive judgments when asked to explain the causes of their judgments and actions. Haidt refers to this as the post hoc problem (Haidt, 2001). If that is correct, it would appear that moral considerations are not conveyed spontaneously without unprompted reflection. Moreover, since different modes of personal reflexivity have been proposed (cf. Caetano, 2015), it is also possible that the servicemen did reflect more upon their behavior than what they shared spontaneously with the interviewers. This might be an alternative explanation for the differences found in moral identity between the participants in a leadership position and a subordinate position since leaders have received more extensive training that helps them to reflect and consequently verbalize their ideas and feelings.

Furthermore, a widely acknowledged issue in interpreting interview data is the degree to which symbolic phenomena are recognized by the researchers when coding the material (cf. Crittenden \& Hill, 1971). Despite the fact that the raters of the material were well informed about the military context and had both engaged in participant observations in differing deployment areas, this could have played a role. Although we believe our approach to have been suitable for the exploratory nature of this study, using multi methods in future research may confirm or refute our findings.

Finally, given the small number of participants in the sample, it is advisable to be cautious with interpretations of these outcomes. This study should be regarded as exploratory, taking a first glance at the moral identity of military personnel in relation to moral disengagement using Blasi's framework.

In addition, the results might also be functionally and culturally biased. In our study, we focused on military personnel who have off-camp duties, such as patrol missions and convoys. For this reason, most of our participants were army or navy (especially marine corps). In view of technological advances, the ethical challenges of air force personnel as well as, for example, on-base medical teams are relevant to address as well. Moreover, we have not addressed the ethical challenges and moral identity of military personnel who remain on camp, such as analysts and staff personnel. 
Furthermore, a cultural bias may have occurred since only Dutch military personnel took part in this study. The extent to which our observations are generalizable to other countries' troops, such as the U.S., whose operations differ enormously, remains an open question. We therefore suggest that future research devote attention to other branches of service as well as other nationalities when addressing moral identity. Another avenue for future research would be to conduct longitudinal research and investigate whether moral identity perception is stable over time, or shifts over time due to (traumatic) life events or other factors.

\subsubsection{Conclusion}

To conclude, this exploratory study appears to have revealed a rather coherent pattern regarding ethical challenges in military deployment that give direction for practice and further research. Although the servicemen consider moral identity and ethical challenges at the heart of their profession, their critical self-reflection and self-assessment seems to be somewhat underdeveloped. Such critical reflection may also prove to be valuable in tackling acts of moral disengagement. That is, our findings suggest that, contraintuitively, a more strongly developed moral identity appears to serve more to initiate moral disengagement processes, rather than inhibit them, presumably to alleviate the discomfort caused by frequent double-bind situations. This is an important finding, because both theory and practice often assume that a strong moral identity could inhibit moral disengagement processes. 



\section{Chapter 4}

\section{Emotional reactions and moral judgment: The effects of}

morally challenging interactions in military operations

This chapter is based on: De Graaff, M. C., Schut, M., Verweij, D. E.M., Vermetten, E., \& Giebels, E. (2016b). Emotional reactions and moral judgment: The effects of morally challenging interactions in military operations. Ethics \& Behavior, 26(1), 14-31.

We thank Karla Boeijen-Mennen, Walter van Bijlevelt, Frank Peters, Matthijs Moorkamp, AnneMarie van Veldhuizen, Sven Zebel, and Sarena Duff for their assistance in the data collection and for their comments on previous versions of this chapter. 
"During a patrol mission in Afghanistan, where I was one of the drivers, we escaped from a suicide attack. A man had blown himself up in a marketplace close by. We are still not sure what his target might have been. Except for him, nobody had died in the incident... Since we were this close to the site, we were commanded to collect body-parts and remains of the bomb. After identification, the body would be returned to his relatives in order to ensure a proper funeral. We all started to collect whatever we could find. However, at one moment I started to realize what I was doing... This man... maybe we had been his target! And now I had to collect body parts so he could get his funeral! It wasn't me who had blown him up in pieces! The whole situation made me sick, I was disgusted by what we had to do. At the same time I felt so angry, I felt he had it coming and I didn't want him to have the honor of a proper funeral. I didn't want to comply with our orders then... but there was not much choice."

(Fragment from an interview with a female Dutch Corporal regarding her deployment experiences.)

This example illustrates the challenging situations that military personnel may encounter during deployment. In this case it involves choosing between the conflicting values of compliance on one side and fairness on the other. It has generally been acknowledged that military deployment comes with such morally challenging interactions (for an overview of differing types of moral challenges, see Richardson, Verweij \& Winslow, 2004). We define morally challenging interactions as situations in which an individual is confronted with an intrapersonal 'clash' of values caused by an interaction with others. Morally challenging interactions may occur in every environment in which individuals interact. However, in some environments these dilemmas are more evident than in others, for example due to cultural diversity and extreme circumstances. We propose that they are particularly likely to occur in the context of military operations because of (a) large cultural differences, (b) a necessity to act, (c) a not self-evident situation, and (d) high stakes (cf. Kramer, 2007). These features create an environment susceptible to extreme, complex and morally challenging situations. Others have referred to such situations as moral dilemmas (e.g., Van Baarda \& Verweij, 2006). Because servicemen do not always perceive a situation as a dilemma but refer to it as an interpersonal frustration, the more neutral term morally challenging interaction is used throughout this article. Confrontations with morally challenging interactions may evoke strong emotions, because deciding what the best course of action to take is often regarded as extremely difficult (Van Baarda \& Verweij, 2006; Verweij, Hofhuis, Soeters, 2007). This is clearly illustrated in the example described above, wherein the 
Dutch Corporal experiences both anger and disgust. In turn, these emotional reactions are likely to influence the (behavioral) response strategies (cf. McCullough, Kilpatrick, Emmons, \& Larson, 2001). For example, anger may trigger aggression, as has been illustrated by incidents such as the My Lai Massacre and more recently the Haditha Incident. In the My Lai Massacre in South Vietnam, 1968, over 400 civilians - including children, women and the elderly - were gang-raped, mutilated and eventually killed by US troops (cf. Oliver, 2003). US Marines killed 24 Iraqi civilians - including children, women, and the elderly - in Haditha, Iraq in 2005 (cf. Knickmeyer, 2006).

The goal of this explorative study is to gain insight in the process of moral judgment in military operations by exploring the relationship between morally challenging interactions and response strategies. We also focus on the possible mediating role of emotions. Since there is a lack of earlier empirical research in this field (cf. De Graaff, Den Besten, Giebels \& Verweij, 2016a), transcripts of interviews with recently deployed Dutch servicemen serve as input for a narrative analysis, based on the grounded theory approach (cf. Corbin \& Strauss, 1990; Glaser \& Strauss, 1967).

This study has both a theoretical and practical ambition in relation to moral judgment during military deployment. Firstly, we aim to increase the understanding of the psychological process of moral judgment in the context of morally challenging and emotionally charged encounters that present themselves during military operations. Due to the exploratory nature of this study, the results give a first glance at the type of interactions servicemen experience and the processes present in these experiences. Furthermore, by exploring different types of morally challenging interactions, we may be able to estimate to what extent this process is contingent upon the specific type of interaction. As such, the outcomes of the study are also likely to have practical value for the deployment of troops by military organizations, as it may result in recommendations for pre-deployment-training programs to better identify risky encounters and to prevent incidents of irresponsible acts from happening.

In the remaining part of the introduction, we will provide an overview of the field on the affective approach in moral judgment research, followed by a theoretical framework that we use as the basis for our analyses.

\subsection{Moral Judgment Research: an Affective Approach}

Historically, studies on moral judgment mainly focused on (1) group dynamical processes, such as obedience and anonymity (e.g., Milgram, Orenstein, \& Zafrir, 1989) and (2) on cognitive processes in individual reasoning following the social cognitive theory (Bandura, Barbanelli, Caprara \& Pastorelli, 1996; Greene \& Haidt, 2002). From 
the 1990s onwards, an affective approach gained more and more attention in the field of moral psychology. This led to an abundance of studies focusing on intuition and emotion in relation to moral judgment (e.g., Greene \& Haidt, 2002; Haidt, 2001; Harris, 2003; Kroll \& Egan, 2004; Tangney, Stuewig, \& Mashek 2007). Research on the incorporation of emotions in moral judgment in the military context is scarce (Schut, De Graaff \& Verweij, 2014). Most studies address emotions in relation to stress or symptoms of PTSD and are mainly of a more clinical nature (e.g., Engelhard, Olatunji \& De Jong, 2001; Friedman, 2006; Litz et al.,1997; Milgram, et al., 1984; Rademaker, Vermetten \& Kleber, 2009; cf. De Graaff et al., 2016a). Moreover, the non-clinical literature focusing on moral judgment within the military is mostly theoretical/conceptual of nature, and not so much empirically driven (e.g., Olsthoorn, 2005; Olsthoorn, Verweij, Meijer, 2010; Richardson, et al., 2004; cf. De Graaff et al., under review). Furthermore, the few empirical studies that were conducted on moral judgments in the military mainly focused on cognitive processes without addressing emotions and intuition (e.g., Olsen, Eid \& Johnson, 2006; Seiler, Fischer \& Voegtli, 2011; Verweij, et al., 2007; Williams, 2010).

Moral emotions can be defined as '[emotions] that are linked to the interests or welfare either of society as a whole or at least of persons other than the judge or agent' (Haidt, 2003, p. 276). Thus, moral emotions reflect basic human emotions (such as anger, compassion and fear) but in a specific context enclosing a third-party aspect that causes a moral challenge (Rozin, Lowery, Imada, Haidt, 1999). These moral emotions are often caused by events in which the social order of human life is inflicted, such as discrimination, or aggression, and which do not necessarily and directly involve the observer him- or herself (Rozin, et al., 1999). Haidt (2003) distinguishes between four categories of moral emotions: 1) other-condemning emotions, 2) self-conscious emotions, 3) other-suffering emotions, and 4) other-praising emotions (see Table 8). In the opening example of this article, the potential harm of the suicide bomber may have led the Corporal to experience feelings of disgust and anger (other-condemning emotions towards the bomber).

\subsection{Moral Judgment Research: Response Strategies}

Emotions may have a prolonged effect (cf. McCullough, Kilpatrick, Emmons \& Larson, 2001; Tangney et al., 2007; Thiel, Connelly \& Griffith, 2011). According to Lerner and Keltner (2001), emotions trigger processes that persist beyond the eliciting situation. These emotion-related processes subsequently direct behavior and cognition in response to objects or events that may only have a slight relation to the original cause of the emotion (Lerner \& Keltner, 2001). 
Table 8 Moral emotions based on Haidt (2003)

\begin{tabular}{|c|c|c|c|}
\hline Main category & Description & Subcategory & Operationalization \\
\hline $\begin{array}{l}\text { Other-condemning } \\
\text { emotions }\end{array}$ & $\begin{array}{l}\text { Encloses feelings that } \\
\text { involve a negative } \\
\text { judgment regarding others. } \\
\text { Sometimes referred to as } \\
\text { the hostility triad (Rozin et } \\
\text { al., 1999). }\end{array}$ & $\begin{array}{l}\text { Anger } \\
\text { Contempt } \\
\text { Disgust }\end{array}$ & $\begin{array}{l}\text { Discusses feelings of frustration, } \\
\text { madness, rage, hatred, disapproval, } \\
\text { anger, contempt, disgust, loathing, } \\
\text { aversion, superiority, etc. in a } \\
\text { moral context. }\end{array}$ \\
\hline $\begin{array}{l}\text { Self-conscious } \\
\text { emotions }\end{array}$ & $\begin{array}{l}\text { Involves ongoing } \\
\text { assessments of the } \\
\text { individual's perception } \\
\text { of his/her personal moral } \\
\text { worth and ability to fit } \\
\text { within a community (Rozin } \\
\text { et al., 1999). }\end{array}$ & $\begin{array}{l}\text { Positive } \\
\text { Pride } \\
\text { Negative } \\
\text { Shame } \\
\text { Embarrassment } \\
\text { Guilt }\end{array}$ & $\begin{array}{l}\text { Discusses feelings of pride, self- } \\
\text { importance, self-satisfaction etc. in } \\
\text { a moral context. } \\
\text { Discusses feelings of shame, } \\
\text { embarrassment, guilt, humiliation, } \\
\text { disgrace, blame, remorse etc. in a } \\
\text { moral context. }\end{array}$ \\
\hline $\begin{array}{l}\text { Other-suffering } \\
\text { emotions }\end{array}$ & $\begin{array}{l}\text { Most strongly felt when } \\
\text { it involves close relatives } \\
\text { (such as one's kin or } \\
\text { friends), but can also be } \\
\text { caused by the suffering } \\
\text { of total strangers (cf. } \\
\text { McCullough et al, 2001). }\end{array}$ & Compassion & $\begin{array}{l}\text { Discusses feelings of compassion, } \\
\text { empathy, sympathy, pity, concern, } \\
\text { care, etc. in a moral context. }\end{array}$ \\
\hline $\begin{array}{l}\text { Other-praising } \\
\text { emotions }\end{array}$ & $\begin{array}{l}\text { Positive emotions that } \\
\text { originate in situations } \\
\text { in which an individual } \\
\text { considers other people's } \\
\text { behavior as virtuous (cf. } \\
\text { Emmons \& Crumpler, } \\
2000 \text { ). }\end{array}$ & $\begin{array}{r}\text { Awe } \\
\text { Gratitude } \\
\text { Elevation }\end{array}$ & $\begin{array}{l}\text { Discusses feelings of awe, } \\
\text { gratitude, elevation, admiration, } \\
\text { respect, appreciation, etc. in a } \\
\text { moral context. }\end{array}$ \\
\hline
\end{tabular}

Research shows, for example, that anger motivates aggressive actions towards the inflictor (cf. Haidt, 2003) or more punitive judgments of others involved in the incident, even when this is not appropriate (cf. Thiel et al., 2011). Individuals deal with their emotions in interpersonal relationships and social interactions by using a certain response strategy (Gable \& Gosnell, 2013). Generally, response strategies in morally challenging interactions can be regarded as a form of coping. Coping refers to an individual's attempt to regulate one's reaction and meet the specific demands of a stressor (cf. Connor-Smith \& Flachsbart, 2007). Although decades of research on coping have identified an abundance of different strategies (for military studies see: Limbert, 2004; Nilsson, Sjöberg, Kallenberg, \& Larsson, 2011; Rademaker et al., 2009), a recent metaanalysis by Connor-Smit and Flachsbart (2007) shows that two global categories of response strategies are distinguished under such conditions. First, individuals may use 
active (or engagement) strategies, involving vigorous attempts to manage the situation at hand. Second, individuals may make use of passive (or disengagement) strategies, for example by avoiding the stressor (Connor-Smith \& Flachsbart, 2007). In order to further categorize active response strategies, we used Bandura's framework of moral disengagement (e.g., Bandura, 1999). We distinguished the passive response strategies into numbing (e.g., Lifton, 1973) and ethical relativism (e.g., Whetham, 2008).

Moral disengagement refers to the process in which the behavior of an individual or a certain group of people - despite their moral self - gradually and often unwittingly becomes irresponsible (Bandura, 1999). According to Bandura, the self-regulatory process of internal moral control permits an individual to selectively activate or withdraw from his or her moral standards (cf. Bandura et al., 1996; Bandura, 1999).

Numbing is a term introduced in the field of psychiatry and refers to the process of de-symbolization and deformation. This refers to a state of mental disturbance: an individual is no longer capable of human mentation (Lifton, 1973). Therefore, the individual is incapable to create and re-create meaningful images and forms in relation to an occurring event (Lifton, 1973). Within the clinical literature similar attempts of suppression are referred to as psychological dissociative defense mechanism, which is strongly related to the well-researched elements of depression and Post Traumatic Stress Disorder (PTSD) (e.g., Nijenhuis \& Van der Hart, 2011). However, discussing the comprehensive theories on depression and PTSD falls outside the scope of this article. Instead, numbing is used (which resembles elements of the mentioned clinical models) as a concept in this study. This is because it is better confined and also well studied within a military context (e.g., Litz, 1992; Litz et al., 1997). Thus, numbness refers to an individual's absence of emotional responsiveness to situations that 'normally' evoke an emotional reaction (Litz, et al., 1997).

Another passive response strategy, ethical relativism, differs from numbing as the servicemen do not 'shut down' their feelings but they tend to relativize and overrationalize the situation at hand (cf. Whetham, 2008). Ethical relativism emerges from '[...] what at first appears to be commonsense position that ethical judgments vary from culture to culture and that therefore there is no objective standard by which to judge a specific act' (Whetham, 2008; p. 305). Thus, in this disengagement strategy the servicemen are aware of their environment and the challenge they face - in contrast to numbing - but they do not act upon these situations since they believe they have no right to do so given the specific cultural determination of the situation.

To summarize, based on theory and previous moral judgment research discussed in the sections above, the model depicted in figure 1 is hypothesized to explain the relations between morally challenging interactions, moral emotions and response strategies. It is assumed that emotions mediate in the relation between morally challenging interactions 
and response strategies during military operations. Table 9 presents the operationalization of the response strategies described in the previous paragraphs.

Table 9 Response strategies based on Bandura (1999), Lifton (1973) and Whetham (2008).

\begin{tabular}{|c|c|c|c|}
\hline \multicolumn{3}{|c|}{ Constructs } & \multirow[b]{2}{*}{ Operationalization } \\
\hline & Main category & Subcategory & \\
\hline $\begin{array}{l}\text { Active } \\
\text { response } \\
\text { strategies }\end{array}$ & $\begin{array}{l}\text { Reconstruction of } \\
\text { own behavior or the } \\
\text { incident }\end{array}$ & Moral justification & $\begin{array}{l}\text { Justifies what happens, for example by } \\
\text { saying that the goals justify the means. }\end{array}$ \\
\hline
\end{tabular}

Advantageous comparison Compares the incident or own behavior with other situations that are considered worse, for example by saying that torture is permitted since the victim killed innocent children.

Euphemistic labeling Uses language that masks what happens, for example discussion collateral damage

Reconstruction of own role in the incident

Displacement of responsibility

Diffusion of responsibility

Dehumanization

Reconstruction of the parties involved
States not to be held responsible for what happened, for example by saying someone else gave an order.

States it is unclear who is responsible in the situation, for example by saying there were others present as well who could have intervened.

Uses language that dehumanizes individuals or groups of people, for example by referring to them as dogs instead of people.

Blaming the victim
States the victim has to blame himself for getting into this situation, for example by saying they started the terrorizing first.

\section{Passive Numbing \\ response \\ Strategies}

Discusses a state of emotional numbness or apathy, for example by saying the situation goes by in a blur.

Puts the situation in perspective, for example by saying that (cultural) differences are omnipresent and improbable to overcome. 


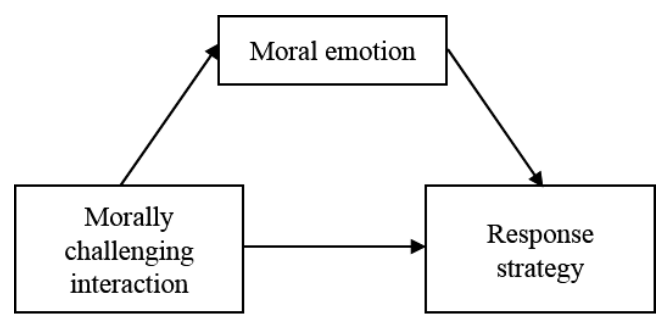

Figure 1 Expected relations between the concepts

\subsection{Method $^{3}$}

For the purpose of this study, transcripts of interviews were content analyzed according to grounded theory approach. The aim of this study was twofold. For the first aim - categorizing the types of moral challenges in military operations - no previous research or theory was used to make constructions of this reality. Here, coding was used as 'an iterative, inductive, yet reductive process that organizes data' (Walker \& Myrick, 2006, p. 549). By means of coding themes, categories of moral challenges could be constructed from the servicemen's narratives. Second, this study aimed to gain insight in the relationship between moral challenges and behavioral responses - in terms of engagement and disengagement strategies - and the mediating role emotions play in this relation. For this purpose, the proposed theoretical framework was used as a starting point for abduction: a qualitative research strategy within grounded theory in which theoretical knowledge and presumptions serve as heuristic tools in order to make sense of social phenomena in social interactions (cf. Richardson \& Kramer, 2006).

\subsubsection{Sample}

Participants were selected from units that experienced direct contact with the local population or coalition forces during a military deployment. First, participants having being deployed in Afghanistan as part of Task Force Uruzgan (under ISAF command from 2006-2010) were interviewed. Additional servicemen participating in other operations were recruited for participation as the process continued in accordance with snowball sampling (Biernacki \& Waldorf, 1981; Noy, 2008). Most participants had been deployed to Afghanistan, others were deployed to countries such as Angola, Iraq, Liberia and Bosnia. The deployment experiences varied between one and five times deployed $(M=2 ; S D=1.2)$. The participants differed in age and rank: varying from 18

\footnotetext{
${ }^{3}$ The methodology described in this chapter is to a large extent similar to the method-section described in the previous chapter. As these chapters have been written as independent studies that have been submitted (and published) to different journals it was decided to maintain/display the original content of both papers in this dissertation.
} 
years to 47 years old ( $M=28$ years; $S D=8.3$ ) and from private/marine up to Lieutenant Colonel. Of the participants, 17 were in a leadership position. Most participants were male. Only 7 women participated in the study, which can be explained by the fact that very few women are enlisted and deployed abroad.

\subsubsection{Procedure}

First, brigade commanders were asked to support this study. They all considered the topics addressed relevant and gave their consent for us to contact their subordinates. Second, 60 individuals were initially personally contacted by E-mail (officers) or by a personal letter to their commanding officers (for troops and NCOs). Participants were given the opportunity to address this call by sending an E-mail to the researchers, or by informing their commanding officer, who in turn would inform the researchers about the servicemen's willingness to participate. The participants were informed that the project aimed at gaining insight into deployment experiences and its impact on the daily life of individual servicemen. All participants were ensured confidentiality, meaning their commanding officers or other third parties would not be notified of their individual answers. A total of 45 servicemen voluntarily agreed to participate in this study, and with them a meeting was arranged. A further 11 servicemen - willing to take part in an interview session - eventually did not participate for differing reasons, such as unavailability or not meeting the research criteria. At the beginning of each interview session the participant was again informed about the procedure and the possibility to abort the session at any time. It was also ensured that those participants who were approached by their commanding officers were truly present on a voluntary basis.

Interview format. Data was collected by qualitative semi-structured interviews following a prepared interview guide. The interview guide was tested in a small number of interviews before using it in the main study in order to ensure all participants were asked similar questions regarding their deployment experiences. Regular informed consent procedure was followed. The participants were all interviewed by two behavioral scientists. One of the interviewers (the first author of this article) was continually present throughout all interview sessions, whereas the second interviewer position was shared between two researchers. The meetings were arranged at a location that suited the participant. Most interviews were carried out near the servicemen's workplace or at their homes. The interview location was quiet and secured from disturbance in order for the participants to speak freely. Therefore no colleagues, commanders, or family members were present or near during the interview sessions, which lasted approximately 50 minutes. The interviews were all digitally recorded and transcribed afterwards.

The interviews consisted of open-ended questions covering basic demographic information (such as rank, age, and professional function) and of individually adapted 
follow-up questions covering the interviewee's general experiences in the mission area. This general introduction was needed in order for the servicemen to construct internalized and evolving stories (narratives) wherein morally challenging situations would be addressed. The participants were invited to share their experiences and narratives, so open-ended questions were asked, such as 'Can you give us an example of a situation that was quite difficult to cope with during deployment?' This gave the researchers the opportunity to go into detail with regard to the respondents' remarks. When respondents did not describe it themselves explicitly, morally challenging situations were addressed with questions like: 'Did you encounter situations in which you were confronted with 'unusual' things?' and, 'Can you explain what you considered unusual in this particular example?' Other questions were: 'Did you engage in behavior you later thought of as condemnable?' and: 'Did you encounter situations in which your own principles or convictions about right or wrong were challenged?' In order to address the emotions experienced in the situations, we elaborated upon the servicemen's narratives and their facial or non-verbal expressions with questions such as: 'This seems to affect you in some way. Can you explain your feelings about this situation a bit further?' When the serviceman gave no hint of having experienced emotions in the specific situation we explicitly asked him/her to reflect on the situation and to think over the emotions that he/she experienced.

Coding procedure. After the interview sessions, the interviews were transcribed and systematically content analyzed by three researchers, two of whom were unaware of the specific research questions. The first author of this article coded all transcripts. For this a manual coding-guide was developed describing the rating strategy, covering the operationalization of each construct (see Table 8 and 9). Markers that highlighted key aspects of the construct, from the responses of the pilot study, represented high agreement with each construct.

First, two transcripts were coded independently by all raters. The goal was to categorize the specific types of morally challenging situations. After coding, the three raters discussed their findings. This led to an abundance of codes that had to be narrowed down to general applicable codes in terms of which other parties were involved in the morally challenging situation. Finally four remaining general codes were added to the manual coding guide: a) local cultural interaction, b) work-home interaction, c) teamrelated interaction, d) coalition force interaction (see for a discussion, the results section).

Then, two raters coded four interviews independently (Kappa: .43) and discussed their findings. It became apparent that at times one of the raters had coded a narrative, whereas the other rater had left the narrative uncoded. Therefore, Kappa showed moderate agreement. It was decided that the rater who had left narratives blank needed to code these specific sections again (unaware of the other rater's codings). This was performed and led to a higher Kappa: .78, which reflects substantial inter-rater 
agreement. For the remaining interview transcriptions this procedure was followed in order to establish agreement about the codes.

\subsection{Results}

This section first provides an analysis of the morally challenging interactions described by the interviewed Dutch military personnel. Then, descriptive data of the measured constructs is reported. Finally, the mediation model is tested on the basis of regression analyses, in line with Baron and Kenny (1986).

\subsubsection{An Overview of the Reported Morally Challenging Interactions}

On the basis of the 'others' involved in the situation, the reported morally challenging situations were categorized as relating to four broad types of ethical challenges: a) local cultural interaction, b) work-home interaction, c) team-related interaction, d) coalition force interaction. Moral challenges in the category of local cultural interactions originate from perceived differences in moral standards between the Dutch forces and local principles or values. Other researchers referred to such situations as morally and culturally critical situations (Schut, et al., 2015). A sergeant, who found himself and his unit in such a delicate situation, described an example of such a challenge: 'When we arrived at a local police station we heard a woman screaming. It turned out she was locked away in the ammo box we installed only a few days before. She was stressed. Without her husband being present, local traditions prohibited the woman from being in a room with other men. So, the policemen locked her up in the ammo box. For us, that caused a dilemma. We didn't want to provoke the policemen or put the woman's honor to shame, but we also wanted to protect her safety and wellbeing and get her out of the box.'

Work-home morally challenging interactions refer to situations that create a moral challenge in the interaction with close relatives, such as spouses, parents, children or other loved ones. An example of a moral challenge in work-home interactions was described by a corporal who experienced a dilemma when communicating with family back home: 'We had experienced quite a lot; several wounded, one of my buddies had died, and I had to collect body-parts of a suicide-bomber... I wanted to share these experiences with my family back home... I needed their comfort and reassurance. However, I knew that when I would tell them what I was facing every day it would hurt them terribly.'

Team-related morally challenging interactions refer to situations in which the serviceman's interaction with direct colleagues - such as unit-members, staff-members or a commander - causes a moral challenge. One of the platoon commanders described how (from his perspective) the military staff obstructed the operation, a challenge 
we categorized as a work-related interaction. He described: 'The staff's perspective was completely off. They used their previous deployment experiences as frame for this operation. However, Afghanistan is quite different from for example former Yugoslavia. So, they continuously asked me to carry out impossible assignments which created for me the dilemma: to obey orders or to assure the wellbeing of my own personnel?'

A fourth category of morally challenging interactions refers to moral challenges caused by cooperation and communication with other coalition forces present in the mission area. A sergeant described an example of a moral challenge in relation with interaction with coalition forces. This sergeant's team was confronted with destructions in a village caused by one of the Western coalition partners: 'It was quite a challenge to explain to the villagers that we were not 'the bad guys'... That we had nothing to do with this. The villagers were frustrated and angry, and the situation got hostile. I understood their reaction, I would probably be angry too, had I been in their situation. The situation remained under control, the villagers cooled down. The next day we had to carry out an assignment together with the coalition partner that had caused the damage to the village. It felt awkward to cooperate with them since I didn't agree with their way of operating and it felt like deceit towards the villagers. However, I had no choice but to deal with it since we needed the coalition forces to complete our own mission.'

\subsubsection{Descriptive Data for our Main Constructs}

All narratives were coded for the operationalization of the different variables, including the types of morally challenging interactions. For each narrative the times a variable was mentioned was determined. The means, standard deviations, and correlation between the variables measured in this study are presented in Table 10.

Table 10 shows that challenges related to team-related interactions were most often mentioned $(M=4.67 ; S D=4.12)$. Least mentioned were coalition force interactions $(M=1.73 ; S D=1.10)$. Other-condemning emotions were the most described moral emotions by the participants $(M=2.98 ; S D=3.36)$. Least described were negative selfconscious emotions $(M=0.20 ; S D=0.51)$. The response strategy servicemen most often reported was relativism $(M=2.53 ; S D=2.69)$, while the least mentioned were diffusion of responsibility $(M=0.04 ; S D=0.21)$ and dehumanization $(M=0.04 ; S D=0.21)$. Advantageous comparison was not expressed at all and is therefore not presented in the table. 


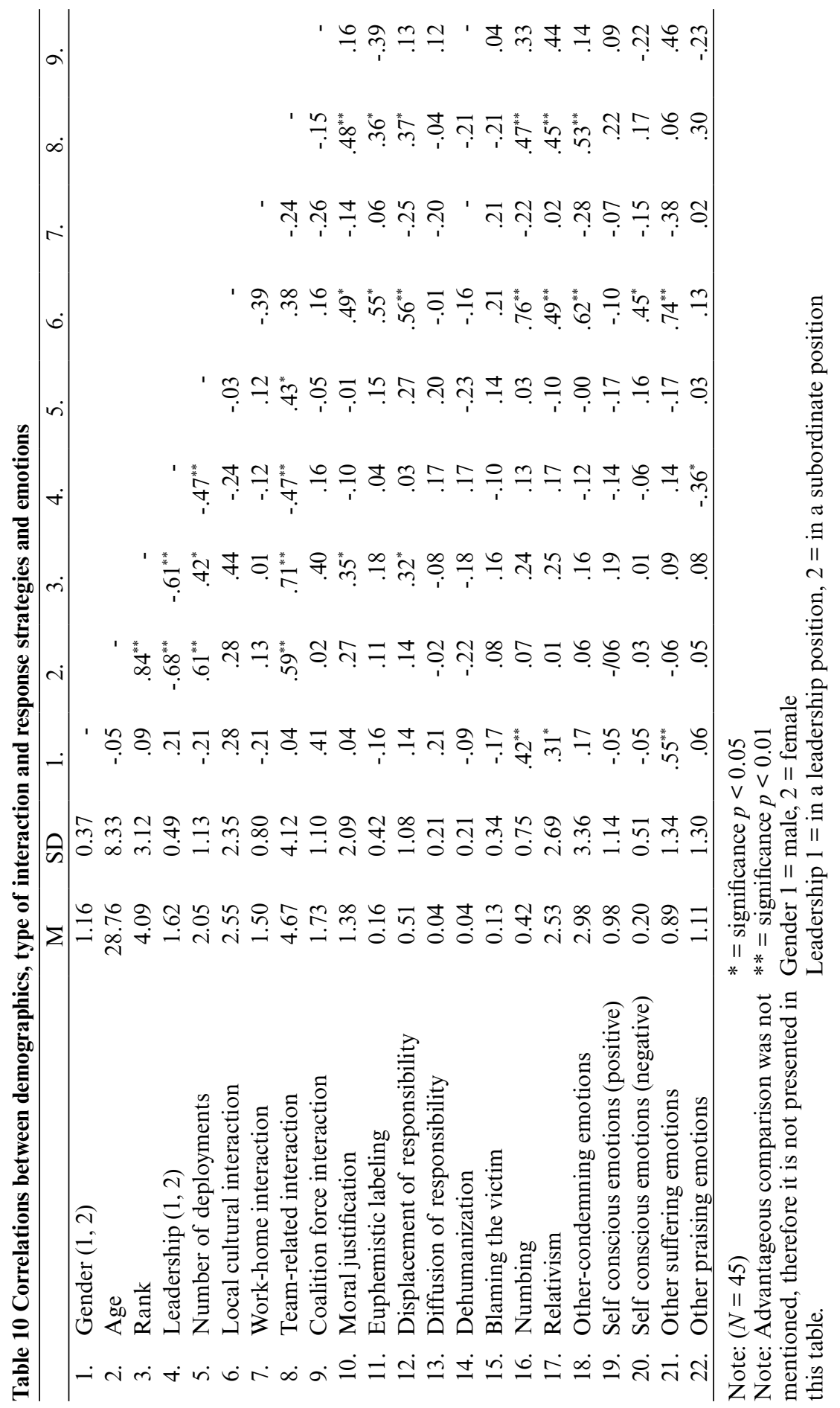




\subsubsection{Correlations}

First, a correlation analysis between morally challenging interactions and response strategies was conducted. This analysis showed significant correlations between both the mentioning of local cultural interactions as well as team-related interactions with the response strategies: moral justification; euphemistic labeling; displacement of responsibility; numbing and relativism (all .36<rs<.76; all $p s<.05$ ). Then it was tested whether the mentioning of specific interactions is correlated with certain emotions. This correlation analysis showed significant correlations between local cultural morally challenging interactions and other-condemning emotions $(r=.62, p<.01)$, negative self-conscious emotions $(r=.45, p<.05)$, and other-suffering emotions $(r=.74, p<$ $.01)$. A significant correlation was also found between team-related morally challenging interactions and other-condemning emotions $(r=.53, p<.01)$.

Several significant correlations between emotions and response strategies were found (see Table 11 for a complete overview of the correlations between these variables). Other-condemning emotions positively correlate with the response strategies: moral justification, numbing and relativism (all $.51<r s<.59$; all $p s<.01$ ). Also, other-suffering emotions positively correlate with these three response strategies and with diffusion of responsibility (all $.33<r s<.57$; all $p s<.05$ ).

Next, we conducted independent sample t-tests in order to examine to what extent military leaders and their subordinates score significantly different on the variables (see Table 12). The correlational analysis showed a relationship between leadership and team related ethical challenges/other praising emotions. The independent sample t-test verified only one these relations. Leaders uttered more other-praising emotions $(M=$ $1.71 ; S D=1.61)$ than their subordinates $(M=.75 ; S D=.93)(t(22.53)=2.23, p<.05)$. No significant differences were found for leadership and the types of ethical challenges, the response strategies, or the other categories of emotions.

\subsubsection{Regression Analyses}

To test our mediation model, we performed a series of regression analyses in which each time a different response strategy was used as the dependent variable. In line with Baron and Kenny's (1986) approach, we only included local-cultural and team-related interactions as predictors as they were the only situations showing a relationship with both emotions and response strategies. In step 1, we entered the control variables rank, gender, age, number of deployments and leadership position, in step 2 we entered the type of moral interaction, and in step 3 we included the emotional category, to examine its possible mediating role. These analyses show that emotions have a mediating effect for certain response strategies. 


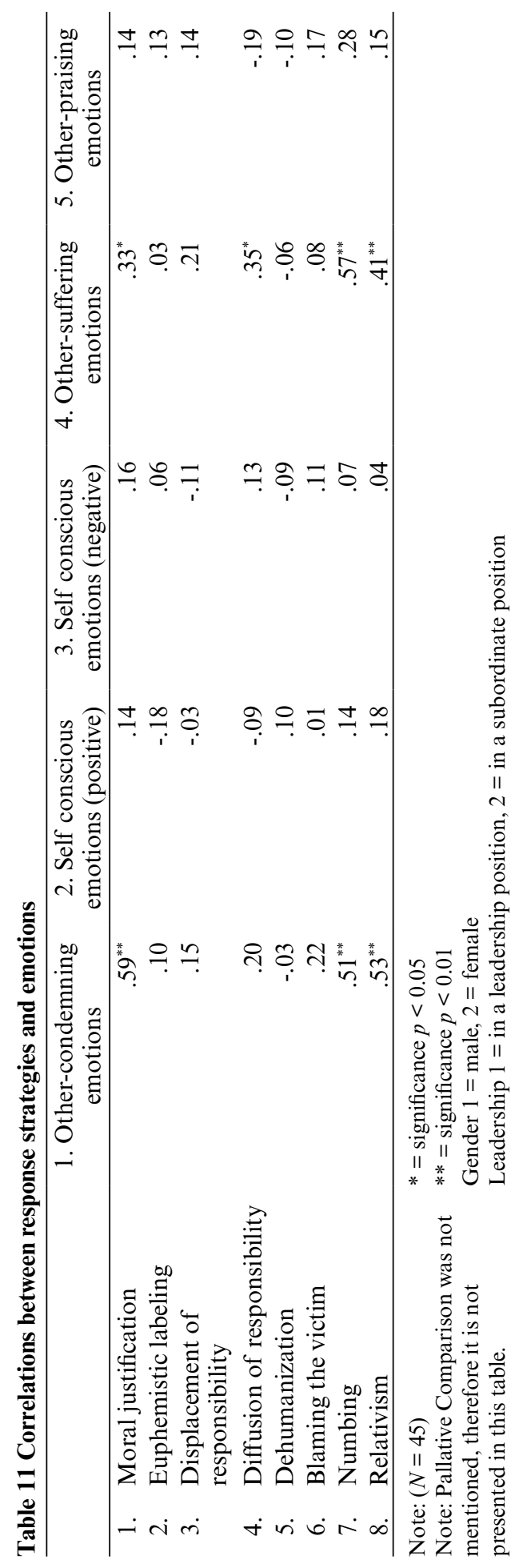


Only other-condemning emotions had a significant mediating-effect on the relation between several types of morally challenging interactions and response strategies. Both the relation between local cultural morally challenging interactions and moral justification $(\mathrm{B}=.26, p \leq 0.05)$ or relativism $(\mathrm{B}=.30, p<0.05)$ is mediated by othercondemning emotions. Also, other-condemning emotions had a significant mediating effect in the relation between work-related morally challenging interactions and the response strategy moral justification $(\mathrm{B}=.33, p<0.01)$. See Tables 13 and 14 for the regression analyses.

Table 12 Means and SDs for main constructs in relation with leadership-position (Yes/No)

\begin{tabular}{lrcccc}
\hline & \multicolumn{2}{c}{ Yes } & \multicolumn{3}{c}{$\begin{array}{c}\text { No } \\
\text { (N=28) }\end{array}$} \\
\hline & Mean) & SD & Mean & SD & t \\
\hline Local cultural ethical challenges & 1.35 & 2.18 & 1.00 & 1.93 & $.57, n s$ \\
Personal ethical challenges & .76 & 1.03 & .71 & .90 & $.17, n s$ \\
Work related ethical challenges & & & & & \\
Team/own troops related & 5.29 & 5.30 & 3.29 & 3.13 & $1.42, n s$ \\
Coalition force related & .35 & .79 & .46 & 1.00 & $-.39, n s$ \\
Other-condemning emotions & 3.47 & 4.46 & 2.68 & 2.56 & $.67, n s$ \\
Other-suffering emotions & .65 & 1.12 & 1.04 & 1.45 & $-.95, n s$ \\
Other-praising emotions & 1.71 & 1.61 & .75 & .93 & $2.23 *$ \\
Self-conscious emotions (positive) & 1.18 & 1.51 & .86 & .85 & $.80, n s$ \\
Self-conscious emotions (negative) & .24 & .56 & .18 & .48 & $.36, n s$ \\
Total number of response strategies & 4.71 & 5.76 & 5.54 & 5.29 & $-.49, n s$ \\
$\quad$ Numbing & .29 & .59 & .50 & .84 & $-.89, n s$ \\
Moral justification & 1.65 & 2.42 & 1.21 & 1.89 & $.67, n s$ \\
Euphemistic labeling & .18 & .39 & .14 & .45 & $.26, n s$ \\
Diffusion of responsibility & .47 & .62 & .54 & 1.29 & $-.19, n s$ \\
Dehumanization & .00 & .00 & .07 & .26 & $-1.12, n s$ \\
Blaming the victim & .00 & .00 & .07 & .26 & $-1.12, n s$ \\
Relativism & .18 & .39 & .11 & .32 & $.65, n s$ \\
Displacement of responsibility & 1.94 & 2.70 & 2.89 & 2.66 & $-1.16, n s$ \\
\hline
\end{tabular}

* significant difference at .05 level

Finally, significance of the indirect path was established by conducting a Sobeltest, using the bootstrapping procedure (Preacher \& Hayes, 2004). These analyses showed the indirect effect to be significant for the relationship between local cultural interactions, other- condemning emotions and moral justification (95\% (CI: .01 - 1.43; 1000 bootstrap resamples) at a significance level of $\mathrm{p}<0.001$. For the relationship between team-related interactions, other-condemning emotions and moral justification the indirect effect showed to be significant at a $p<0.01$ level (95\% (CI: -.28 - 1.34; 1000 bootstrap resamples). 


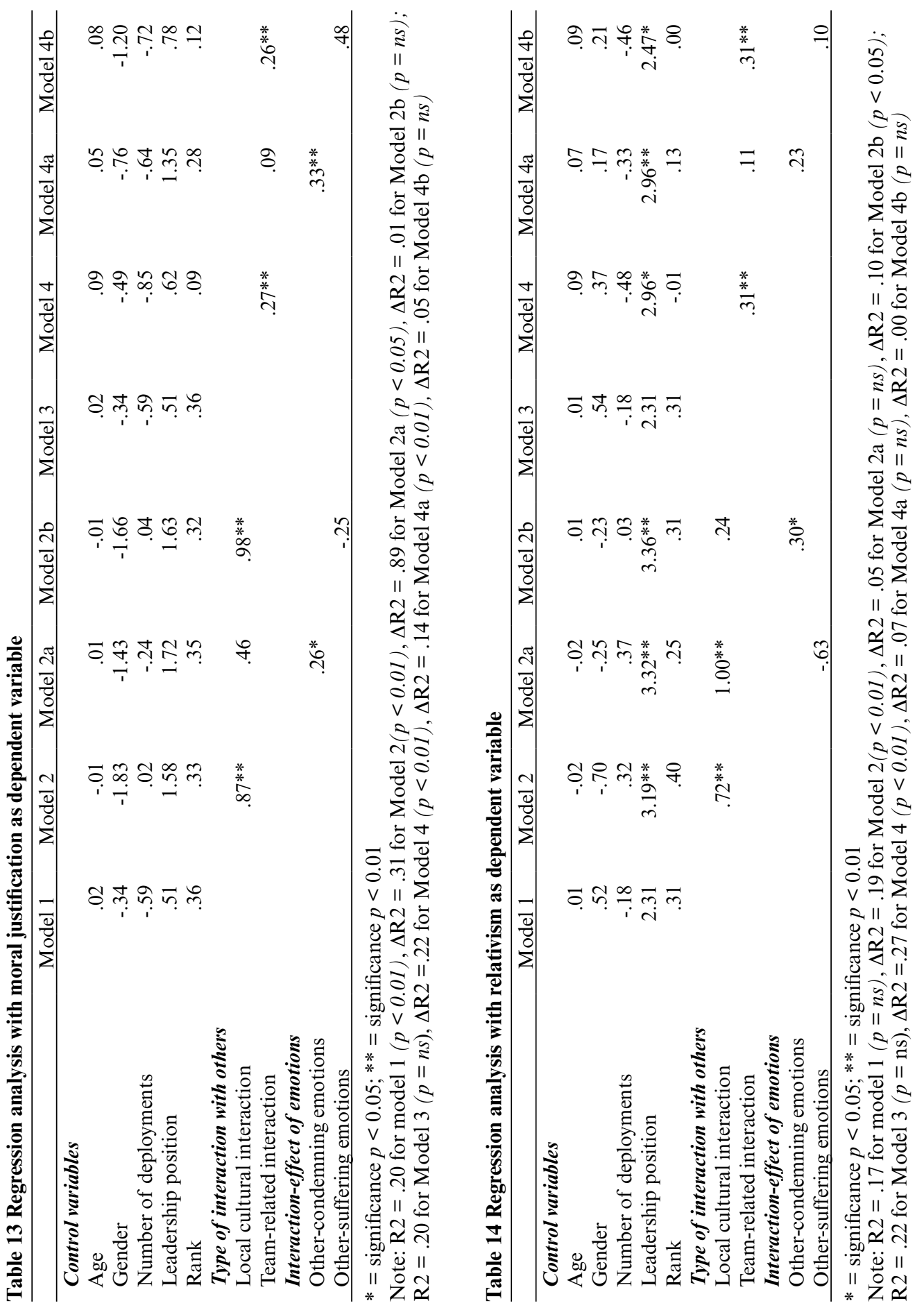


This was also the case for the relationship between local cultural interactions, othercondemning emotions and relativism (95\% (CI: .01 - .58; 1000 bootstrap resamples). Therefore, the prediction that other-condemning emotions mediate the relationship between local-cultural interactions and moral justification or relativism is supported. As is the prediction that other-condemning emotions mediate the relationship between work-related interactions and moral justification.

\subsection{Conclusion and Discussion}

In the present study, we explored the presence of emotions in morally challenging interactions related to military deployment and their mediating role in the relation between these interactions and the following (behavioral) response strategies. This study offers three main conclusions.

First, four broad types of morally challenging interactions can be distinguished in relation with military deployment (i.e., local cultural; work-home; team-related and coalition force interactions). Team-related morally challenging interactions were most described, followed by local-cultural morally challenging interactions.

Second, the present study shows that the participating servicemen report using relativism most often as a response strategy in morally challenging interactions. In relation with local cultural interactions, servicemen express that they find themselves in a different culture where they should respect common traditions. However, doing so may lead to what Schut and Moelker (2014) refer to as moral incapacity since one's own values are sidelined or neglected in that situation. Also, the response strategies moral justification, numbing and relativism showed a strong relationship with local cultural and team-related morally challenging interactions.

Third, the findings of this study indicate that other-condemning emotions are most brought up by the servicemen in differing morally challenging interactions. Also, strong correlations between local cultural morally challenging interactions and emotions, as well as strong correlations between work-related morally challenging interactions and emotions were found. The results addressing the mediator effect of emotions revealed that only the relations between local cultural or team-related interactions and moral justification, and between local cultural interactions and relativism proved to be significantly mediated by other-condemning emotions.

\subsubsection{Limitations and Future Research}

While offering several interesting suggestions about emotions experienced in relation with military deployment, and their influence on response strategies in 
morally challenging interactions, this exploratory study also has limitations. In order to explore moral judgment, narratives of servicemen were used and consequently interpreted. Table 8 and 9 presented the operationalization of concepts, which should be used as a means to interpret the material as objectively as possible. The described operationalization should be regarded as sensitizing concepts. These give direction to researchers' focus and are a suggestion of the participants' construction of their social reality by helping the researchers understand the participant's situation and feelings (cf. Blumer, 1969; Schwartz \& Jacobs, 1979). In explorative/qualitative research this is common methodology. The next step is to use the categorization of morally challenging interactions that we propose, with the findings regarding moral emotions and response strategies as a framework or a starting point for further exploration and validation. In order to do so, quantitative screening methods should be used during the interviewsessions and when analyzing the material.

Recent studies pointed out the relevance of judgment difficulty and moral intensity for ethical decision-making (cf. Sparks \& Siemens, 2014). Also, moral judgment research showed that certain morally challenging situations evoke more intense emotional processing than others (cf. Greene \& Haidt, 2002). Therefore, we suggest to address these features of morally challenging interactions (such as proximity and intensity), in relation to emotional reactions during military operations in future research. After all, research suggests a strong link between moral intensity and mental health problems such as PTSD and moral injury (Litz et al., 2009).

Earlier research regarding moral judgment indicates differences between individuals' emotional responses to morally challenging situations (e.g., Fumagalli, et al., 2010; Gibbs, Arnold \& Burkhart, 1984; Hannah, 2013). Differences were found for example in the success at interpreting others' emotional expressions and also in the degree of expressivity of emotions between men and women (cf. Gross \& Levenson, 1993; You, Maeda \& Bebeau, 2011). Due to the small sample we cannot present strong insights regarding how moral emotions and response strategies correlate with leadership or gender. However, small significant effects of gender and leadership-position were found in our study as well (see Table 10). We would suggest for future research to further elaborate on how this correlates with emotions and response strategies in extremely complex and stressful morally challenging interactions.

\subsubsection{Conclusion}

Since complexity of today's military operations are growing, confrontations with morally challenging interactions are inevitable (De Graaff \& Van den Berg, 2010). It is important to address the servicemen's responses in morally challenging interactions since when it comes to their own behavior; individuals tend to reaffirm their moral 
self-concept even when they have engaged in immoral acts (Harkrider et al., 2013). Subsequently, this moral cleansing can result in engaging in pro-social behavior (e.g., helping others in order to relieve oneself of feeling guilt), or it can license individuals to engage in future immoral acts by self-indulgent behavior (Harkrider et al., 2013). Within a military context this may result in undesirable (side) effects since people's wellbeing and political systems are at stake (cf. Richardson et al., 2004; Seiler et al., 2011). The results of this exploratory study show that the responses of servicemen are mediated by emotions in certain morally challenging interactions. The present study suggests that experiencing emotions and using different response strategies is not exclusive to local cultural morally challenging interactions, but is also related to other types of morally challenging interactions. Until now, intercultural (problematic) interactions had received little attention (e.g., Schut et al., 2014; Schut \& Moelker, 2014). Our study suggests that interactions for example within work units, or between family members can also become morally challenging. The findings of this exploratory study highlight the need for future research with a broader scope beyond intercultural interactions.

Finally it is worth to briefly consider the policy implications of our findings. Within the military, ethical training mainly focuses on teaching rational decision-making models in a high stake environment like deployment (cf. Seiler et al., 2011). However, our findings are a reminder that emotions - regardless of intensity - are inevitable in morally challenging interactions, and influence a serviceman's behavioral responses. Therefore the military would benefit from expanding its attention in the direction of affective processes (De Graaff \& Van Gils, 2012). This would stimulate a professional culture in which recognition of, and adequate coping with, emotions in moral challenges are considered relevant. As such, the military guards its employees from moral injury and other parties from incidents with negative consequences.

Nevertheless, caution should be taken when interpreting the results of this study. After all, this study was conducted as an explorative study giving a first glance at the processes at work and proposing a framework of types of morally challenging interactions. We urge other researchers to further analyze what is occurring in battlefield ethics. Additionally, the field needs to expand beyond the 'military gates', since moral challenges are not exclusive for this domain. It is important that links are established between the military, humanitarian aid workers, social workers, law enforcement officers and medical professionals, in order to cooperate and learn from each other's policy, training and care/administration-system regarding coping with morally challenging interactions. In the public domain, some doubt is cast upon the relevance of emotions and ethics in some professions. Our findings are a strong reminder that morally challenging interactions and the consequent processes cannot remain neglected, as Van Baarda and Verweij (2006) have already highlighted in their appeal for stimulation of moral competence in the military. 



\section{Chapter 5}

\section{Sensemaking in military critical incidents:}

The impact of moral intensity

This chapter is based on: De Graaff, M.C., Giebels, E., Meijer, D., \& Verweij, D.E.M. (forthcoming). Sensemaking in military critical incidents: The impact of moral intensity. Business \& Society.

We would like to thank Sarena Duff, Marije Bakker, Femke Hilverda and Derek Suchard for their contributions to previous drafts of this study. 
When facing ambiguous circumstances or a novel, challenging situation, individuals usually try to figure out what is going on and what the proper response in this situation might be (cf. Weick, Sutcliffe \& Obstfeld, 2005). This cognitive process is labeled sensemaking (cf. Mumford et al., 2008; Weick, 1995; Weick et al., 2005). Sensemaking is particularly important in high-velocity environments for emergency response units such as fire crews (Landgren, 2005) and the subject of this study - the military (Kramer, Bezooijen \& Delahaij, 2010). When such units encounter unanticipated events, referred to as critical incidents, it is crucial that they make the right decision and act responsibly (Alexander \& Klein, 2001; Oliver \& Roos, 2003).

In critical incidents individuals often come across ethical challenges that are characterized by ambiguousness and complexity and that are in demand of sensemaking (cf. Thiel, Bagdasarov, Harkrider, Johnson \& Mumford, 2012). Ethical challenges typically occur when the situation presents a difficult decision caused by the conflicting nature of (or values at stake within) that specific situation (cf. Sparks \& Siemens, 2014). This includes, for example, situations where the obligations to oneself are incompatible with those to someone else, or when benefit for one party results in harm for others (cf. Sparks \& Siemens, 2014). In a military context, for example, this may occur when (radio) contact is lost between units during a patrol mission, while loud noises suggest that one of the other units might be in trouble. The question rises whether to follow the regular guidelines and orders not to intervene, or whether to go and see if the other unit needs assistance. Such dilemmatic and challenging situations usually cannot merely be dealt with on the basis of regular routines or rules; they require ethical decision-making (EDM) competence also referred to as moral competence (Seiler, Fischer \& Voegtli, 2011; Parks \& Peterson, 2006).

\subsection{Research Aims}

Research shows that when complexity increases, decision-making comes about differently (cf. Cummings \& Nehme, 2010) and affects performance (cf. Alexander \& Klein, 2001). Generally, when the situation becomes more complex and loses its resemblance to what is expected or trained for, it becomes hard to construct reality (Weick, 1993). Weick (1993) demonstrated for example that in a huge fire incident in 1949 called the Mann Gulch disaster the firefighter-crew was not able to make sense of the situation since their 'old labels' did not suffice in the changing environment, resulting in the tragic death of almost all firefighters (Weick, 1993).

Moreover, scholars have highlighted the influence of issue- and content-specific characteristics on decision-making (cf. Caughron et al., 2011; Christensen \& Kohls, 
2003; Weber \& Wasieleski, 2001). Jones (1991) refers to the characteristics of situations - such as the harmfulness (to a larger group of people), the likeliness to occur, and the direct impact on or personal relevance for the decision-maker - as the moral intensity of a situation. It has been generally acknowledged that military deployment comes with ethical challenges that differ in moral intensity (for an overview, see Richardson, Verweij $\&$ Winslow, 2004). There is - to our knowledge - no empirical research regarding how moral intensity precisely affects the sensemaking process for active military personnel. This study aims to fill this void. Therefore, this study's first aim is to explore whether and how sensemaking differs in coping with low and high moral intensity situations.

Secondly, since earlier work suggests that the different sub dimensions of moral intensity are not equally important in assessing a situation (Singh et al., 2007; Singhapakdi et al., 1996), we aim to further explore the moral intensity construct and its' relationship with sensemaking. Thus, a) we address how the moral intensity sub dimensions differentiate between situations that are experienced as low in intensity versus high in intensity. And b), we explore the effect of these sub dimensions on the use of different sensemaking tactics.

In sum, this study contributes to a better understanding of sensemaking in ethical challenges in relation with moral intensity and its dimensions. These insights are relevant for organizations that operate in high velocity environments, since when individuals are not capable of making sense of their surroundings they are not able to create order and give meaning to what occurs. Consequently, there is nothing to decide and actions are not formed by deliberate moral reasoning, which may have negative and even lethal consequences. Also, ethical decision making (EDM) depends on personal as well as situational characteristics (Treviño, 1986), meaning that the level of moral development does not form moral judgments exclusively. Therefore, it is important for individuals to show internal awareness (i.e., awareness of their own thoughts, feelings and biases) and external awareness (i.e., awareness of what is happening in the environment) before they continue with actual decision-making. Thus, in ethical challenges it is relevant to address how moral intensity influences individual sensemaking. After all, insufficient sensemaking may result in unethical behavior regardless of an individual's personal level of moral development.

\subsection{Theoretical Background}

The scientific field that primarily addresses dilemmas and ethics in a work context is that of business ethics wherein various academic disciplines (such as psychology, organizational science and philosophy) are combined. Efforts to understand processes 
surrounding EDM have generally taken one of two perspectives. The first perspective is normative of nature and focusses mainly on normative organizational ethics, such as behavioral codes and business ethos (cf. O'Fallon \& Butterfield, 2005). The second perspective seeks to understand how EDM develops at a personal and individual level, tapping into issues such as moral reasoning, moral development and individual behavior (cf. O'Fallon \& Butterfield, 2005). In this article, we take the second of these approaches, referring to the general field of ethical decision making.

Three main approaches with regard to individual judgment are present in EDMresearch: 1) the cognitive or rationalist approach, 2) the affective approach, or 3) the integrative approach, combining cognitive and affective elements into sensemaking processes (cf. Kahneman \& Sunstein, 2005). The cognitive approach explains people's EDM by individual cognitive sophistication (Sonenshein, 2007). The affective approach takes intuitions and emotions in consideration when confronting an ethical situation (Greene \& Haidt, 2002). The debate whether to use a cognitive or affective approach in EDM-research led to the third approach wherein both perspectives are united into one integrative approach where interpretations of the world are done by both conscious deliberation and affect-laden intuitions (cf. Lerner \& Keltner, 2001).

Influential frameworks for individual EDM research are Rest's four components model (Rest, 1986) and Treviño's person-situation interactionist model (Treviño, 1986). Rest (1986) proposed a fundamental model for EDM, including four stages: a) moral recognition, b) moral evaluation or judgment, c) moral intention, and d) moral behavior. Where Rest (1986) focusses on individual characteristics in EDM, Treviño (1986) points out the relevance of situational characteristics that influence an individual. According to Treviño (1986) it is insufficient to focus only on individual moral development when addressing EDM. Treviño argues that an individual's moral development forms the basis for an individual's response to a moral dilemma, however they are not enough to explain or predict EDM behavior (Treviño, 1986). Such situational variables include job characteristics, responsibility and pressures (Treviño, 1986). Recent research suggests that issue-related factors, like moral intensity, are strong (and sometimes even stronger) predictors of EDM than personal and organizational factors (Paolillo and Vitell, 2002).

\subsubsection{Moral Intensity}

Moral intensity fits the person-situation interactionist approach suggested by Treviño (1986), as it refers to the degree to which there exists a moral imperative in a situation that influences individual EDM (cf. Jones, 1991; Hayibor \& Wasieleski, 2009; McMahon \& Harvey, 2006). According to the seminal work of Jones (1991), moral intensity is a multi-dimensional construct including six dimensions: a) magnitude of the consequences which is the total sum of harm of the decision, b) probability of effect which refers to 
the likelihood of harm caused by the decision, c) proximity which is the social distance between the decision-maker and other beneficiaries, d) concentration of the effect which refers to the number of people affected by the decision, e) temporal immediacy which is the amount of time between making the decision and the onset of any consequences, and f) social consensus which refers to the extent of broad social agreement about the appropriateness of the decision (cf. Jones, 1991; Paolillo \& Vitell, 2002).

\subsubsection{Sensemaking}

Sensemaking is a process that begins when an individual is confronted with a situation that is new and not self-evident because it deviates from the normal daily routine (Weick, 1995). Usually, this becomes apparent when comparing the current situation with prior experiences and expectations. Furthermore, available mental models are used in order to interpret the situation. Finally, information is gathered, evaluated and assessed so that the individual can figure out how to react. The process is concluded when individuals believe to understand the novel situation well enough to decide how to act (cf. Caughron et al., 2011; Weick et al., 2005). Recently, research has focused on sensemaking in relation to EDM (e.g., Brock, et al., 2008; Martin, et al., 2011; Mumford, et al., 2008). Research shows that the sensemaking process includes a variety of metacognitive reasoning strategies. Caughron et al. (2011) described a categorization in which a person distinguishes between seven different sensemaking tactics: dealing with emotions, recognizing the circumstances (i.e., creating a clear picture of the relevant aspects of the situation by analyzing its origin and the interests of all parties involved), seeking outside help, questioning own and others' judgment, anticipating consequences of actions, analyzing own personal motivations, and considering the effects of actions on others. Others have integrated the tactical level of sensemaking in higher-level strategies, which broadly encompass four broad categories: emotion regulation, forecasting, self-reflection, and information integration (cf. Brock et al., 2008; Thiel, Bagdasarov, Harkrider, Johnson \& Mumford, 2012). We use this integrated framework, outlined and further operationalized in Table 15, as the basis of our research.

Over the last two decades, empirical studies started to examine the relationship between moral intensity and EDM. Most of these studies were conducted in a marketing or business management setting (e.g., May \& Pauli, 2002; Paolillo \& Vitell, 2002; Singhapkdi et al., 1996). In a study with business school undergraduate students, May and Pauli (2002) grouped empirical studies of moral intensity into four broad categories that relate to different stages of EDM: a) moral recognition, b) moral evaluation, c) moral intention, and d) issue-contingent EDM framework (i.e., focusing upon the entire framework proposed by Jones (1991)). They found that moral intensity relates to the recognition of a moral issue. They also showed that moral evaluations of the situation 
and individual moral intentions are related to the moral intensity of the managerial issues the business students are exposed to. Singhapakdi et al. (1996) also pointed at the relevance of moral intensity for the recognition of a marketing dilemma, showing that situations with higher moral intensity are perceived more often as an ethical dilemma than those with low perceived moral intensity. Finally, several studies suggest that not all underlying dimensions of morality are equally important for ethical judgment and behavioral intentions (e.g., Barnett, 2001; McMahon \& Harvey, 2006; Singhapakdi et al., 1996). Barnett (2001), for example, found that social consensus influences the recognition of an ethical issue, whereas behavioral intentions and the final judgment are influenced by social consensus, temporal immediacy, seriousness of consequences, and proximity. Barnett (2001) furthermore reported that temporal immediacy and proximity did not influence the ethical decision-making process at all.

Moreover, McMahon and Harvey (2006) found that when measuring moral intensity with a 12-item scale covering the original six dimensions of Jones (1991), moral intensity is better reflected with three rather than six dimensions (i.e., probable magnitude of consequences, proximity, and social consensus). Singhapakdi and colleagues found that moral intensity was even better represented by two dimensions (Singhapakdi et al., 1996), which they labeled perceived potential harm and perceived social pressure (combining the proximity and social consensus dimensions). They also found that Jones' proximity dimension is a loosely connected component of moral intensity in relation with assessing the ethicality of the situation and the behavioral intentions of the decisionmaker (Singhapakdi et al., 1996).

In sum, this collection of studies suggests that (1) the moral intensity of the issue may have a strong influence on EDM processes including sensemaking in the early EDM stages of moral recognition, and (2) that the specific relationship is likely to vary for the different moral intensity dimensions. However, as far as we know this has never been studied in the context of military deployment nor has it specifically focused on different sensemaking tactics. Specifically, the integrated framework for sensemaking as well as the moral intensity approach from an issue-contingency-model further build upon the integrative approach of EDM research.

\subsection{Method}

In order to address the research aims, this study consisted of two parts. First, actual narratives of military personnel were used in order to select a prototypical high and a low moral intensity critical incident. Second, a scenario study was conducted with active duty military personnel in order to investigate the relationship between moral intensity (high versus low) and the use of sensemaking tactics. 
Table 15 Operationalization of sensemaking based on Caughron et al. (2011) and Thiel et al. (2012)

\begin{tabular}{|c|c|c|}
\hline Strategy & Tactic & Operationalization in Item \\
\hline $\begin{array}{l}\text { 1. Emotion } \\
\text { regulation }\end{array}$ & $\begin{array}{l}\text { Downgrading emotional } \\
\text { overwhelming / dealing } \\
\text { with emotions }\end{array}$ & I attempt to control my emotions strongly \\
\hline \multirow[t]{3}{*}{ 2. Self-reflection } & Introspection & I consciously draw on my own previous experiences \\
\hline & Moral awareness & $\begin{array}{l}\text { I consciously deal with the situation as a moral } \\
\text { dilemma }\end{array}$ \\
\hline & $\begin{array}{l}\text { Analyzing personal } \\
\text { motivations }\end{array}$ & $\begin{array}{l}\text { I am aware of and reflect on my own bias and } \\
\text { prejudices }\end{array}$ \\
\hline 3. Forecasting & $\begin{array}{l}\text { Forecasting the } \\
\text { consequences }\end{array}$ & $\begin{array}{l}\text { I anticipate the consequences of actions for those } \\
\text { involved }\end{array}$ \\
\hline \multirow[t]{3}{*}{$\begin{array}{l}\text { 4. Integration of } \\
\text { information }\end{array}$} & $\begin{array}{l}\text { Recognizing the } \\
\text { circumstances }\end{array}$ & $\begin{array}{l}\text { I aim at creating a clear picture of the } \\
\text { circumstances and its origin: I am mindful of } \\
\text { others' interests in the situation }\end{array}$ \\
\hline & Seeking outside help & I seek help from others in order to advise me \\
\hline & $\begin{array}{l}\text { Analyze and integrate } \\
\text { available information }\end{array}$ & I consider the 'bigger picture' of a situation \\
\hline
\end{tabular}

Note: the items described in this table are a translation of the Dutch items presented in the survey.

\subsubsection{Selection of Narratives}

From a previously collected sample of 45 interviews conducted with servicemen regarding their deployment experiences (De Graaff et al., 2016b), twelve narratives including an ethical challenge were selected (see Table 16 for a short description of the narratives). The content of the narratives show an appropriate distribution across four different types of ethical challenges encountered in the mission area (cf. De Graaff et al., 2016b).

A total of ten servicemen from different Arms of Service were provided with the twelve narratives - each handed out on a separate paper and in random order. They were asked to read the narratives thoroughly. Next, they were asked to rate the narrative in terms of intensity by sorting them from 1 (most intense) to 12 (least intense). Next, the participants were asked to indicate whether they had read narratives they did not find credible (i.e., realistic, plausible). The narratives that were considered not credible by more than $50 \%$ of the participants were excluded from further analysis. From the remaining six narratives, the two narratives that received the highest and lowest average score on intensity were selected for the main study (See table 16). Please note that although the two narratives differed in terms of type (team-related versus local cultural interaction), they were similar in length, type of combat environment -a platoon patrol mission in Afghanistan-, and in both situations another person experienced physical harm (see Textbox 1 for the complete two narratives). 
Textbox 1 Scenarios used in the study

\section{High moral intense scenario}

On a regular patrol mission in Afghanistan, one of the troops gets injured. The medic is called in for assistance. Since the troops were on a foot-patrol in the mountains, the medical vehicle cannot get near the injured serviceman. So, the medic needs to decide - without having precise insight in the severity of the serviceman's injuries; what to carry along going up the mountain? On the one hand, the medical team wants to bring all medical equipment available with them in order to be prepared for a worst-case scenario. On the other hand, because the environment is quite hostile, they are reluctant to do so because they will have to carry their own personal safety equipment as well. The medic needs to decide whether to leave equipment - and if so what should be left behind -, since bringing all medical and all personal safety equipment means that they will be (too) heavily loaded which is therefore no option...

\section{Low moral intense scenario}

There is a meeting of the village-elderly. A force protection team guards and protects this site. Local children make contact and start playing around the military vehicles. One of the children comes too close and tries to take equipment from the vehicles. This is not only prohibited but also very dangerous for the child. The servicemen try to make this clear to the children, without any effect; they keep playing nearby the vehicles and materials. One of the local men, who understands what is going on, heads towards the children and beats one of them with a stick. The Dutch are surprised by this reaction, which is according to the man the regular way to discipline children and he allows the servicemen to do the same when he is again in the meeting. This puts the servicemen in a difficult situation. The local norms differ from their own. According to the servicemen's principles it's wrong to discipline children physically, but they need to keep the children away from the military equipment and they do seem to respond to the local ways...

\subsubsection{Scenario Study}

Procedure and respondents. Servicemen were recruited through contact with the chain of command. The researchers personally administered the hardcopy surveys and the link to the online survey to the participants, in order to assure complete confidentiality to the participants as well as to ensure the voluntariness of their participation. More participants were recruited as the process continued in accordance with snowball sampling (Biernacki \& Waldorf, 1981; Noy, 2008). Participants were asked to forward the link of the online-survey to their colleagues. 


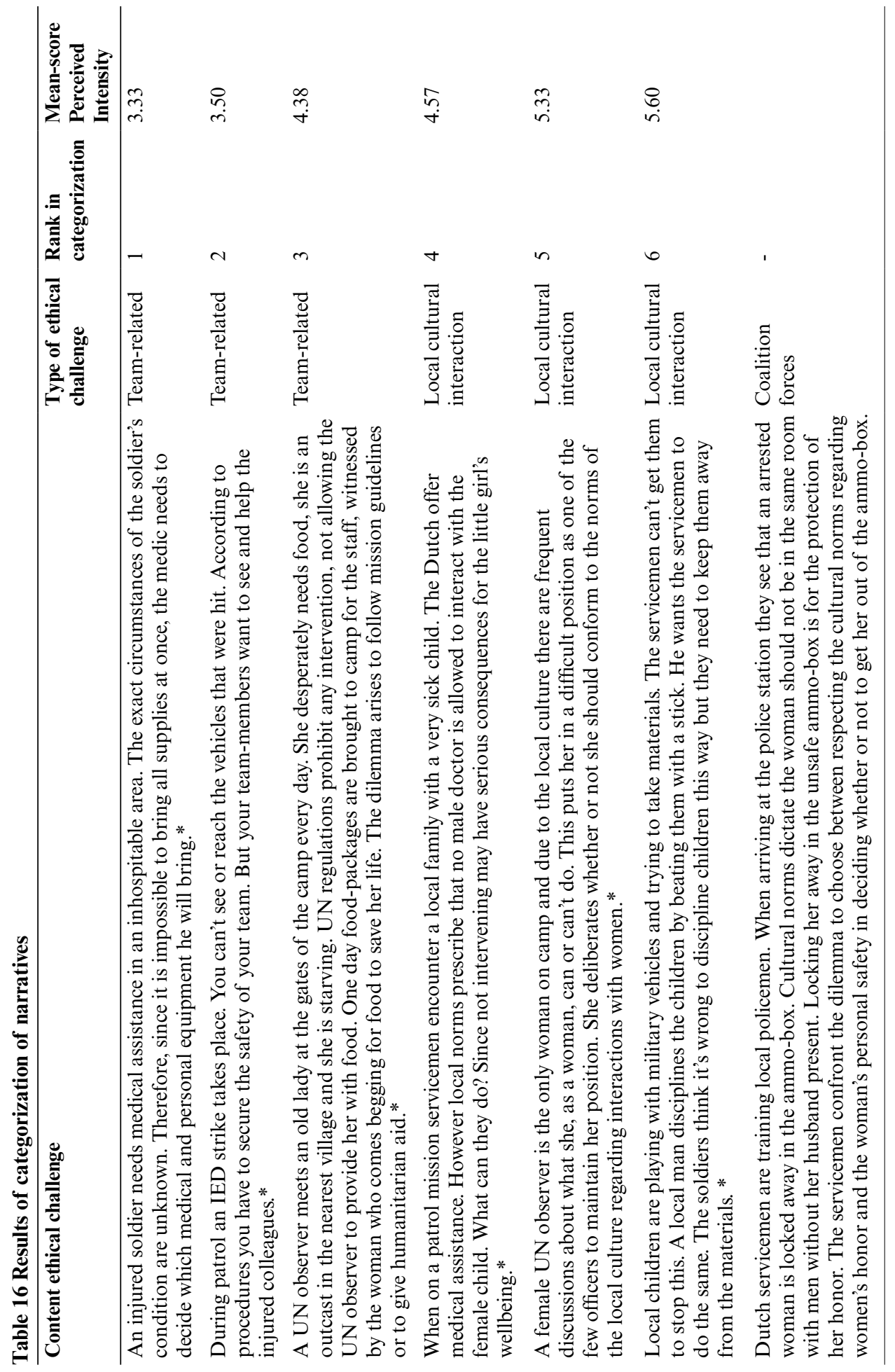




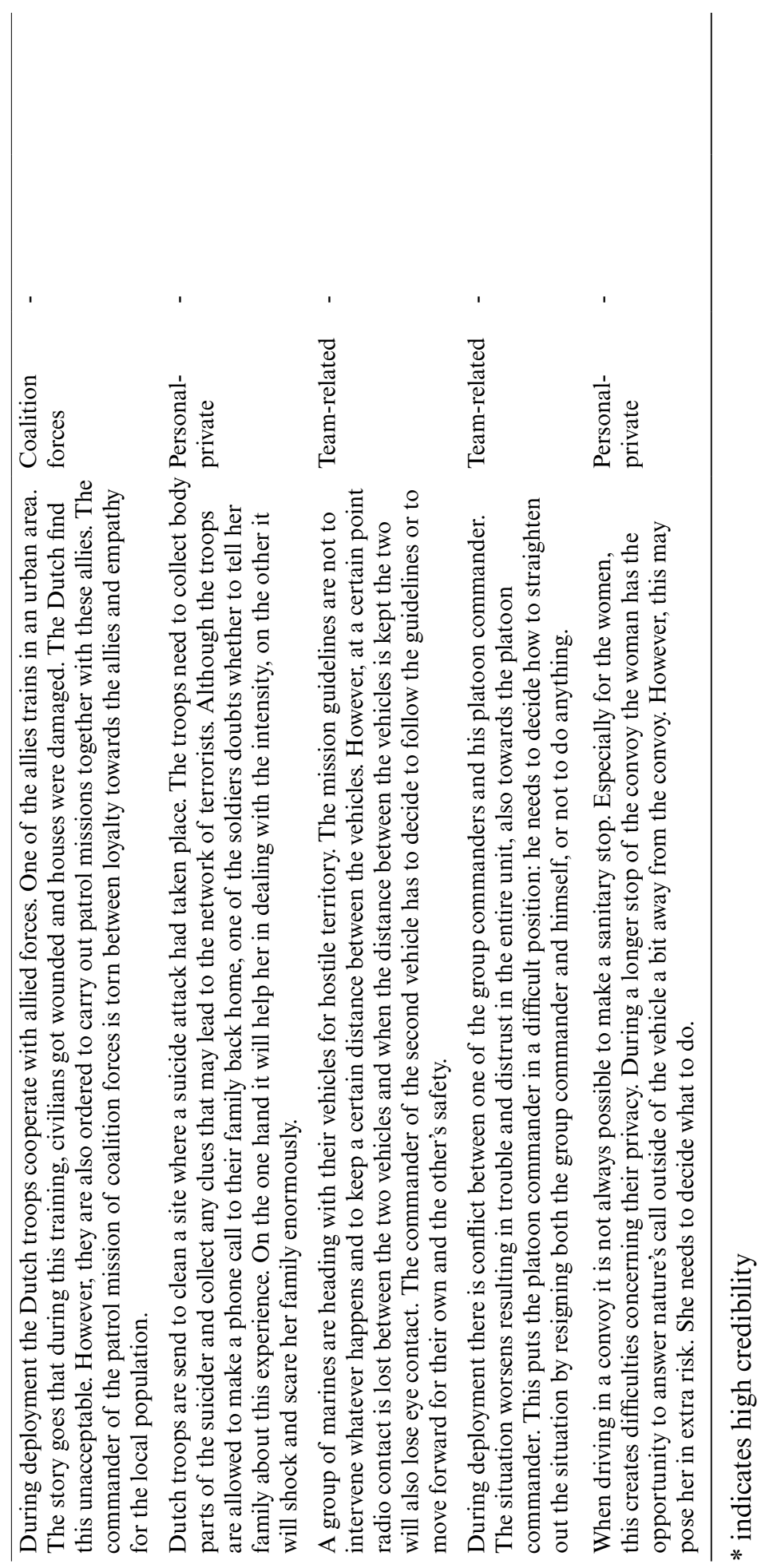


In total 363 participants returned the survey. However, some participants only filled out the questions regarding their demographics, or they did not meet the inclusion criteria (i.e., they were not in active military service at the time of this study). After excluding these participants, a total of 325 surveys were ultimately included for further analysis (see Table 17 for the demographics). Due to missing values, the sample size and the degrees of freedom vary to some extent between the analyses. As is the case in the general military population, the number of women participants was modest $(\mathrm{N}=30$, $9.2 \%$; in the general population of the Dutch forces the percentage of female servicemen centers around 10\%, Ministerie van Defensie, 2015). In our study the number of troops participating $(\mathrm{N}=125,38.5 \%)$ is a larger percentage than in the general population of Dutch forces (which centers around 32\%), as well as the number of officers participating $(\mathrm{N}=31,31 \%$ ), which is also a larger percentage than in the general population of Dutch forces (which centers around 22\%). This difference can be explained by the fact that in military operational units preparing for deployment the number of officers and troops is higher than in non-operational units (such as staff and educational departments). Also, current military operations (one of the criteria for participation in this study) are often individual or team-missions in an international context, which require the rank of an officer. Thus, the distribution of participants is similar to the demographics of the Dutch Armed Forces when focusing on operational and mission-units.

The participants were informed that the study they participated in, aimed at gaining insight into deployment experiences. All participants were ensured confidentiality, meaning their commanding officers or other third parties would not be notified of their participation and their individual answers. The participants were informed about the duration of filling out the survey and the possibility to abort the session at any time. Standard informed consent procedure was followed.

Participants were randomly assigned to one of two versions of a survey starting with either a low or a high intensity moral situation. After the introduction of the scenario the participants were asked to take the perspective of the decision-maker in the narrative. First the perceived moral intensity was measured. Next, we established their preference for the different sensemaking tactics. We concluded the questionnaire with some general questions to check whether participants took part seriously and whether they correctly understood the task ${ }^{4}$.

\footnotetext{
${ }^{4}$ Please note that the entire questionnaire was in Dutch; for this research, all material was translated in English and checked by a native speaker.
} 
Table 17 Demographics of participants

\begin{tabular}{|c|c|}
\hline & $\mathrm{N}=325$ \\
\hline \multicolumn{2}{|l|}{ Gender } \\
\hline Male & 295 \\
\hline Female & 30 \\
\hline \multicolumn{2}{|l|}{ Version } \\
\hline High intensity & 159 \\
\hline Low intensity & 166 \\
\hline \multicolumn{2}{|l|}{ Age } \\
\hline $18-25$ years & 91 \\
\hline $26-35$ years & 115 \\
\hline $36-45$ years & 51 \\
\hline $46-55$ years & 63 \\
\hline $56-65$ years & 5 \\
\hline \multicolumn{2}{|l|}{ In a leadership position } \\
\hline Yes & 125 \\
\hline No & 200 \\
\hline \multicolumn{2}{|l|}{ Number of deployments } \\
\hline None & 117 \\
\hline 1 tour & 66 \\
\hline 1-4 tours & 117 \\
\hline 5 or more tours & 24 \\
\hline \multicolumn{2}{|l|}{ Rank } \\
\hline Private & 79 \\
\hline Corporal & 46 \\
\hline Sergeant & 50 \\
\hline Sergeant-Major & 22 \\
\hline Warrant-Officer & 26 \\
\hline Second Lieutenant & 9 \\
\hline First Lieutenant & 27 \\
\hline Captain & 24 \\
\hline Major & 24 \\
\hline Lt Colonel & 17 \\
\hline Colonel & 1 \\
\hline Average years of service & 13 years (range: $0.5-40$ years) \\
\hline
\end{tabular}

\subsubsection{Measurement}

Moral intensity scale. We assessed moral intensity with a 12-item 6-point Likert scale originally developed by Frey (2000a; see table 4 for the items). Frey's scale to measure Moral Intensity has been used frequently over the years (e.g., Frey, 2000b; McMahon \& Harvey, 2006/2007; Tillman, Hood, Lawrence \& Kacmar, 2014) and is based on the theoretical foundations of Jones (1991). A higher score indicates higher perceived moral intensity. The reliability analysis showed a sufficient Cronbach's Alpha of .65 for the entire scale. 
Table 18 Operationalization of Moral Intensity based on Jones' moral intensity dimensions (1991)

\begin{tabular}{|c|c|c|}
\hline $\begin{array}{l}\text { Moral intensity } \\
\text { dimension }\end{array}$ & $\begin{array}{l}\text { Scale } \\
\text { number }\end{array}$ & Operationalization in Item \\
\hline $\begin{array}{l}\text { 1. Magnitude of } \\
\text { consequences }\end{array}$ & $\begin{array}{l}\text { M1 } \\
\text { M7 }\end{array}$ & $\begin{array}{l}\text { Any negative consequences will be serious. } \\
\text { Harm caused by the decision will be very small. (R) }\end{array}$ \\
\hline $\begin{array}{l}\text { 2. Probability of } \\
\text { effect }\end{array}$ & $\begin{array}{l}\text { M3 } \\
\text { M9 }\end{array}$ & $\begin{array}{l}\text { There is a small likelihood of harm. (R) } \\
\text { The decision is likely to cause harm. }\end{array}$ \\
\hline $\begin{array}{l}\text { 3. Temporal } \\
\text { immediacy }\end{array}$ & $\begin{array}{l}\text { M4 } \\
\text { M10 }\end{array}$ & $\begin{array}{l}\text { The decision will not cause harm in immediate future. (R) } \\
\text { Negative effects will be felt very quickly. }\end{array}$ \\
\hline 4. Proximity & $\begin{array}{l}\text { M6 } \\
\text { M12 }\end{array}$ & $\begin{array}{l}\text { Harmful effects will affect people close. } \\
\text { The decision maker is unlikely to be close to anyone who might be } \\
\text { negatively affected by the decision. (R) }\end{array}$ \\
\hline $\begin{array}{l}\text { 5. Social } \\
\text { Consensus }\end{array}$ & $\begin{array}{l}\text { M2 } \\
\text { M8 }\end{array}$ & $\begin{array}{l}\text { Most people would agree about the appropriate solution. } \\
\text { People are not likely to agree about the right thing to do. (R) }\end{array}$ \\
\hline $\begin{array}{l}\text { 6. Concentration } \\
\text { of effect }\end{array}$ & $\begin{array}{l}\text { M5 } \\
\text { M11 }\end{array}$ & $\begin{array}{l}\text { The harmful consequences of the decision will be concentrated on } \\
\text { a small number of people. } \\
\text { Negative effects of the decision will have large impact on only a } \\
\text { few individuals. }\end{array}$ \\
\hline
\end{tabular}

$(\mathrm{R})=$ reversed score

Note: a higher score indicates a lower perceived intensity; the items presented in this table are a translation of the Dutch items presented in the survey

High versus low moral intensity. We checked whether the high and low moral intensity narratives were actually perceived as distinctive by comparing mean scores of the moral intensity scale. The results indeed showed a significant difference between the high ( $M$ $=3.95, S D=.50)$ and low $(M=3.61, S D=.59)$ moral intensity versions $(t=5.46 ; p<$ $.001 ; d f=295)$.

Sensemaking. The eight sensemaking tactics nested in the four broader strategies were operationalized in eight descriptions of each tactic based on Caughron et al. (2011) and Thiel et al., (2012; See table 15). We offered the respondents forced-choice options of two tactics at a time such that all 28 possible combinations were offered once and in a random order. We chose to use forced-choice rating scales in order to minimize the problem of social desirability bias since pilot testing showed that all tactics are considered important. Earlier studies also indicated that forced-choice scales are less susceptible to response bias than a single-stimulus format (cf. Jackson, Wroblewski \& Ashton, 2000). Each time, the respondents chose the tactic that they were most likely to adopt. Per tactic, the number of choices was added up, with scores varying from 0 (never chosen) to 7 (always chosen), thus reflecting the relative likelihood of each tactic to be used. 


\section{$5.4 \quad$ Results}

\subsubsection{Sensemaking}

The first research question of this study concerns the relationship between moral intensity and the use of different sensemaking tactics. We conducted independent t-tests for each sensemaking tactic grouped by version (i.e., high or low; see Table 19). Of the eight sensemaking tactics, seven showed an effect of high/low moral intensity. Introspection, forecasting the consequences, recognizing the circumstances and seeking outside help were considered more relevant in the high intense situation than in the low intense situation. On the other hand analyze and integrate available information, moral awareness and analyzing personal motivations showed higher scores in the low intense situation, which implies that they are considered more relevant in the low intense situation than in the high intense situation. In the discussion we will further elaborate on these findings. We also examined whether leaders and subordinates scored differently on the differing sensemaking tactics. In the low moral intensity situation leaders scored higher on the dimension 'probability of harm' $(t(92.98)=-2.58, p<.05)$ and used the tactic 'seeking outside help' $(\mathrm{t}(153)=2.216, p<.05)$ less often than their subordinates. In the high moral intensity situation leaders less often used the tactic 'downgrading emotional overwhelming' $(t(141)=2.17, p<.05)$ and more often 'analyzing and integrating available information' $(t(141)=-2.44, p<.05)$ than their subordinates.

Table 19 Means and SDs for Sensemaking Tactics

\begin{tabular}{llcccc}
\hline & \multicolumn{2}{c}{ High } & \multicolumn{3}{c}{ Low } \\
Sensemaking Tactic & Mean & SD & Mean & SD & t \\
\hline $\begin{array}{l}\text { Downgrading emotional overwhelming / } \\
\text { dealing with emotions }\end{array}$ & 2.43 & 1.89 & 2.57 & 1.94 & $-.63, n s$ \\
Introspection & & & & & \\
Forecasting the consequences & 4.64 & 1.65 & 4.09 & 2.05 & $2.54^{*}$ \\
Analyze and integrate available information & 4.37 & 1.62 & 4.00 & 1.64 & $1.97^{*}$ \\
Recognizing the circumstances & 2.99 & 1.81 & 4.25 & 1.98 & $-5.69^{* * *}$ \\
Seeking outside help & 5.39 & 1.48 & 4.32 & 1.66 & $5.86^{* * *}$ \\
Moral awareness & 3.08 & 1.63 & 2.26 & 1.87 & $4.02^{* * *}$ \\
Analyzing personal motivations & 2.52 & 1.75 & 3.59 & 2.01 & $-4.88^{* * *}$ \\
& 1.68 & 1.51 & 2.63 & 1.66 & $-5.13^{* * *}$ \\
\hline
\end{tabular}

* significant difference at .05 level

*** significant difference at .001 level

$\mathrm{N}($ high $)=143 \mathrm{~N}($ low $)=155$ 


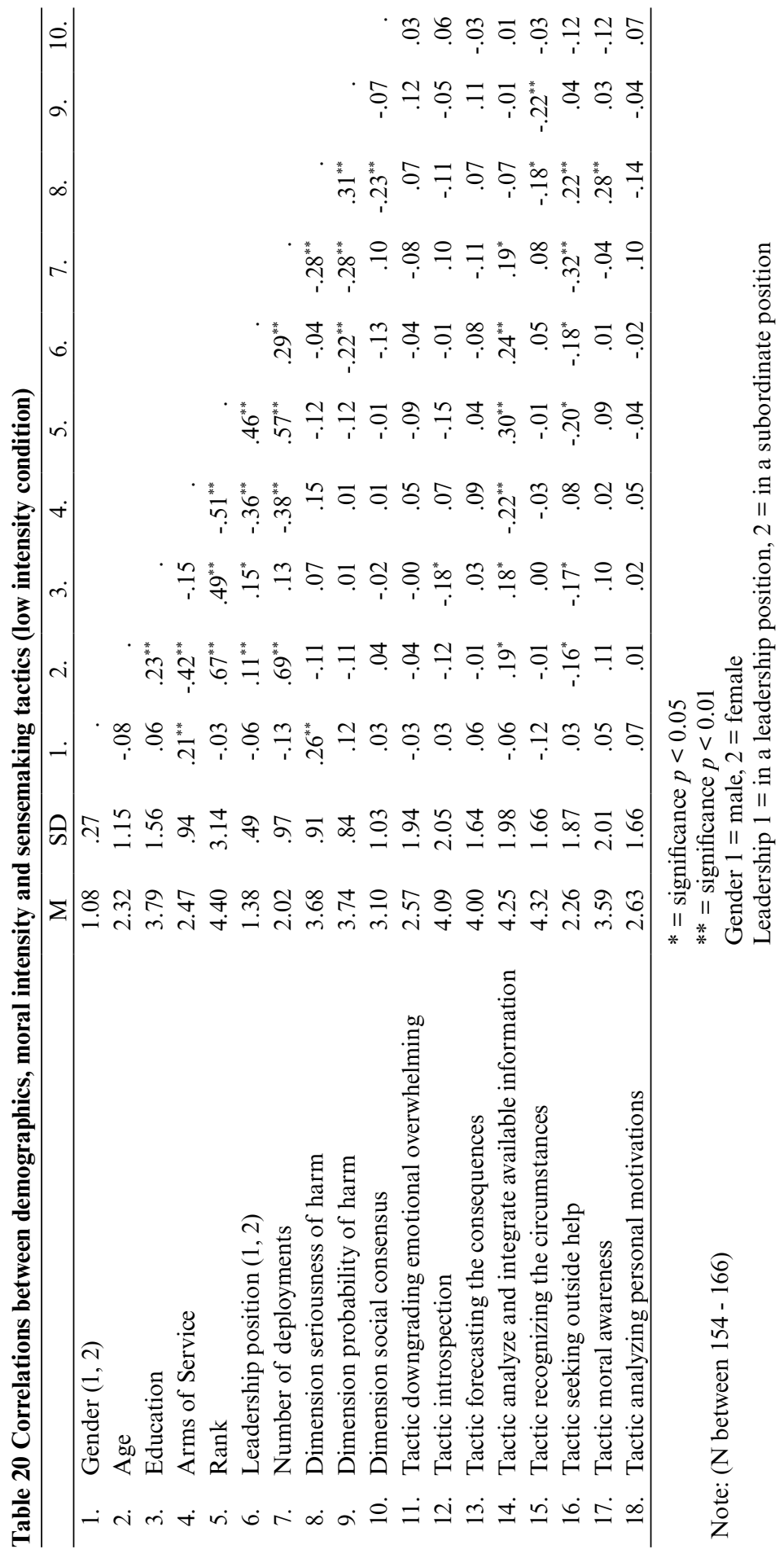




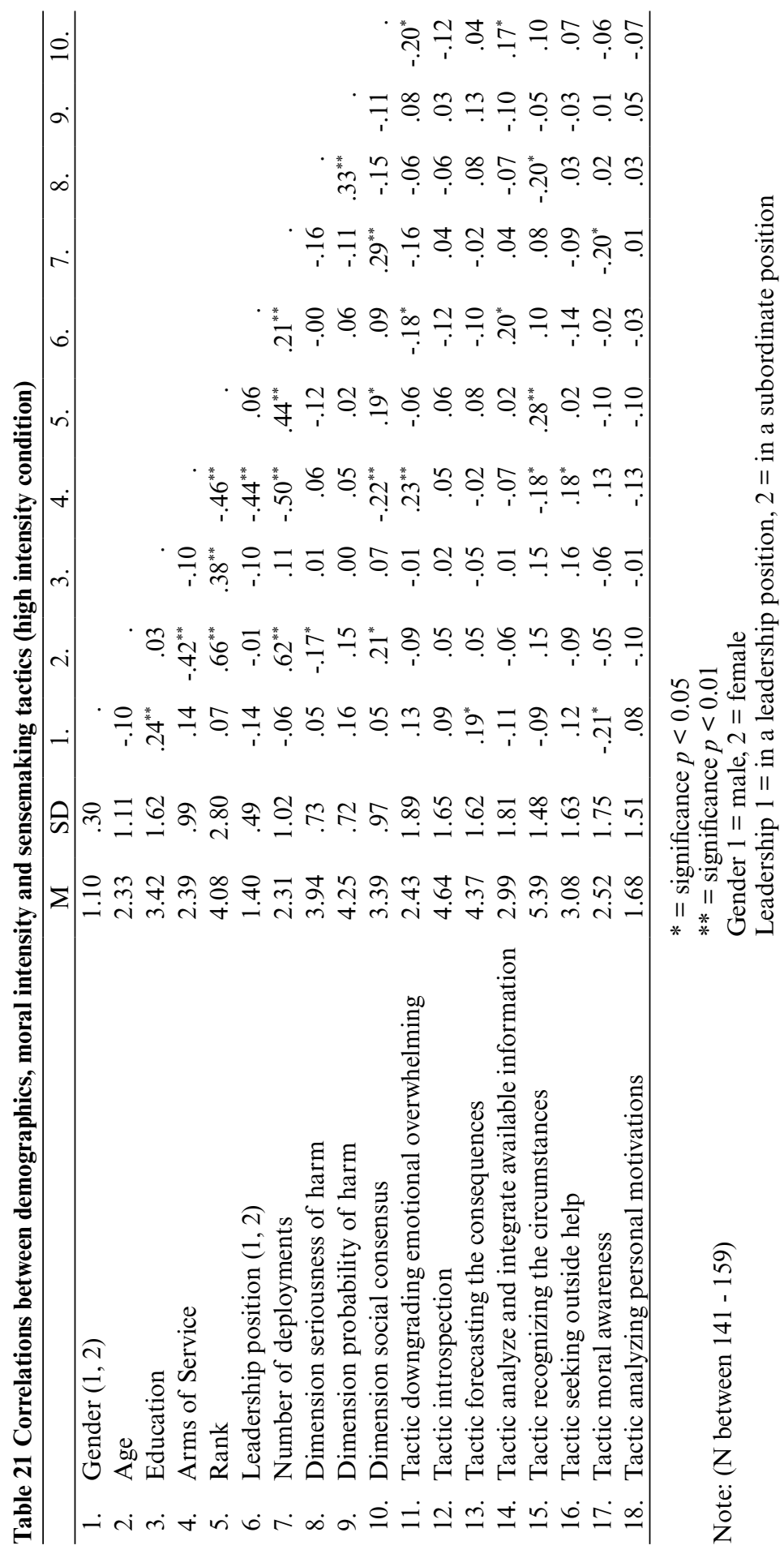




\subsubsection{Moral Intensity Dimensionality}

Although the moral intensity scale displayed a sufficient Cronbach's alpha of .65, an additional aim of this study was to explore a possible dimensionality of moral intensity. Therefore, a factor analysis was performed in order to establish which dimensions (components) of moral intensity can be meaningfully distinguished between. Three components were found, similar to findings of McMahon and Harvey (2006). The first component comprises items reflecting the seriousness of harm in the situation at hand (items: M1; M5; M6; M10; M9; M11; Cronbach's alpha = .74). The second component focuses on the probability of harm in the situation (items: M3; M4; M7; M12; Cronbach's alpha $=.53)$. The third component comprises the social consensus regarding the ethicality of the situation and the decision to be made (items: M2; M8; Cronbach's alpha: .38).

In order to gain insight in the relevance and significance of the different elements of moral intensity in the high and low intense situation, independent sample t-tests were performed at the dimension-level. Not surprisingly, the results show that the seriousness of harm was perceived to be higher in the high $(M=3.94, S D=.73)$ versus low morality condition $(M=3.68, S D=.91 ; t(289.85)=2.69, p<.01)$. A significant difference was also found for the probability of harm, which was higher in the high morality condition $(M=4.25, S D=.72)$ than in the low morality condition $(M=3.74, S D=.84 ; t$ (293) $=5.58, p<.001)$. Similar results were found for the dimension social consensus, wherein the high intense situation showed higher scores for social consensus $(M=3.39, S D=$ $.97)$ than the low intense situation $(M=3.10, S D=1.03 ; t(295)=2.44, p<.05)$. So, all three dimensions are important discriminators of moral intensity.

\subsubsection{Correlational Analyses}

The correlation analyses are shown in Table 20 and 21, separately for the low moral intensity and high moral intensity condition. Most apparent are the positive correlations in the low intensity condition between gender and the dimension seriousness of harm (females $>$ males), and between the number of deployments and the first two moral intensity dimensions. The tactic analyze and integrate available information correlates positively with almost all demographic variables. No correlations between gender and this tactic were found in the low intensity condition. Thus, we found that the older, the higher in rank and the more educated the more the servicemen report the tactic analyze and integrate available information. The tactic recognizing the circumstances negatively correlates with the moral intensity dimensions probability of harm and seriousness of harm. So, the higher the perceived intensity on these dimensions, the lower the reports of this sensemaking tactic. The tactics seeking outside help and moral awareness are positively associated with the moral intensity dimension seriousness of harm. Meaning we found that the more scores for perceived 'seriousness of harm', the more reports 
of seeking outside help and moral awareness. No correlations were found between the dimension social consensus and the sensemaking tactics in the low intensity situation.

In the high intensity condition the following patterns were found. The number of deployments correlates positively with the moral intensity dimension social consensus and negatively with the sensemaking tactic moral awareness. The dimension seriousness of harm correlates negatively with the sensemaking tactic recognizing the circumstances. The dimension social consensus correlates negatively with the sensemaking tactic downgrading emotional reactions and positively with analyzing and integrating available information. No correlations were found for the dimension probability of harm.

\subsubsection{Regression Analyses}

Of the demographic characteristics we were particularly interested in the relationship between the number of deployments reported and perceived moral intensity. These relationships could differ for the low and high intensity scenario, so we performed separate regression analyses to test this. In the low intensity condition we found that when the number of deployments increases, the perceived seriousness of harm decreases $(\mathrm{B}=-.63 ; t=-3.16 ; p<.05)(\mathrm{R} 2$ change $=.06, \mathrm{~F}=3.94)$, as well as the probability of harm $(\mathrm{B}=-.42 ; t=-2.24 ; p<.05)(\mathrm{R} 2$ change $=.03, \mathrm{~F}=2.55)$. Interestingly, no relationships were found between the number of deployments and the intensity dimensions in the high intensity condition. We will address these findings further in the discussion.

The next step was to explore how the different dimensions of moral intensity influence the use of the different sensemaking tactics in both high and low perceived moral intensity situations. In order to do so a linear regression analysis was conducted, for the high and low moral intensity condition separately. In step 1, we entered the control variables rank, gender, age, number of deployments, education, Arms of Service and leadership position. In step 2 we entered the dimensions of moral intensity, to examine whether these dimensions are possible predictors for the use of certain sensemaking tactics. For every single sensemaking tactic a regression analyses was conducted.

These analyses displayed that in the low intense situation, the dimension seriousness of harm is a (marginally) significant predictor of the tactics seeking outside help $(B=.33$; $t=1.82 ; p=.07)(\mathrm{R} 2$ change $=.03, \mathrm{~F}=3.13)$ and moral awareness $(B=.58 ; t=2.84, p<$ $.01)(\mathrm{R} 2 \mathrm{change}=.06, \mathrm{~F}=1.72)$. Finally, the dimension probability of harm is in the low intensity condition a significant predictor of the tactic recognizing the circumstances ( $B$ $=-.35 ; t=-1.98 ; p=.05)(\mathrm{R} 2$ change $=.052, \mathrm{~F}=1.16)$. In the high intensity situation no significant effects were found. 


\subsection{Discussion}

In the current study, we explored the relationship between the moral intensity of military critical incidents and sensemaking. The results are relevant since they extend our knowledge about moral intensity and sensemaking in high-stake environments. This study offers three main conclusions.

First and foremost, we found clear evidence that the overall intensity (i.e., high or low) of a military critical incident influences the use of sensemaking tactics. Only for downgrading emotional reactions no significant effect of the overall intensity of the situation was found. An explanation for this lack of effect may be the servicemen's military training: much effort goes to emotion regulation (cf. Schut, De Graaff \& Verweij, 2015). So, the troops are trained to use this tactic no matter what the intensity of the situation is. Significant differences in the use of certain sensemaking tactics between high and low intensity situations were found for the remaining tactics. The results suggest that in high intense situations the servicemen particularly draw on previous experiences in order to recognize and interpret the situation. Also, they attempt to predict the consequences of their actions, and ask others for help. On the other hand, less attention goes out to higher level 'critical thinking'; moral awareness, integrating available information, and analyzing personal biases in the decision making process in these high intense situations. Thus, it seems that in these ethical challenges the servicemen react by using an analytical manner of assessing the situation without giving room for thorough consideration and doubt regarding how their own reasoning process may cloud their judgments.

Second, our regression analyses reveal that in the low intensity situation the number of experienced deployments have predictive value for the moral intensity dimensions. Interestingly, as the number of deployments increases the situation is perceived as less harmful and serious. We believe that there are two possible explanations for these effects. For one, the servicemen may be emotionally numbed by previous encountered critical incidents (cf. Litz, et al., 1997). However, since no effect was found for the number of deployments on the perceived intensity in the high intensity situation this explanation seems unlikely. Another explanation may be that because of previous experiences the servicemen make a more objective assessment of the critical incident than their less experienced colleagues. It makes sense that this effect is only found in the low intensity conditions as it has been generally acknowledged that under stressful circumstances the decision maker's attention tends to narrow to the most salient issues (cf. Christensen \& Kohls, 2003). Thus, in the high intensity situation it is clear that the issue is salient, whereas in the low intensity situation the salience of the ethical challenge can fall from sight. However, the results of our regression analyses indicate that when the number of deployments increases the use of moral awareness decreases. 
Moreover, the results of the regression analyses regarding sensemaking in the low intensity condition show a link between the presence of the moral intensity dimensions and the use of the tactics recognizing the circumstances, seeking outside help, and moral awareness. For the low intensity condition, the results show that when the perceived seriousness of harm increases seeking outside help and moral awareness are more reported. Also in the low intensity condition we found that when the perceived probability of harm increases the tactic recognizing the circumstances is reported less. These results are interesting since not all sensemaking tactics are linked to the moral intensity dimensions, even though there is an association between the high/low condition and the reporting of almost all tactics. These results suggest that next to the proposed dimensions of moral intensity by Jones (1991) there are other factors that play a part in the assessment of overall moral intensity. For example, De Graaff, et al., (2016b) already pointed at the effect of different types of interaction on emotional reactions in ethically challenging situations. In short, it is possible that the authentic critical incidents used in this study focus on different features that contribute to the overall perception of moral intensity but are not incorporated in the intensity dimensions. This is in line with previous inconsistency in findings regarding the relevance of moral intensity and its sub-dimensions in earlier studies (cf. Elm \& Radin, 2012). Furthermore, Thompson, Thompson and Adams (2008) found that, next to the moral intensity dimensions, aspects such as 'sense of own honor' and 'feelings of pride' regarding personal actions in the situation also play a role in ethical challenges in a military context. Moreover, Blais and Thompson (2008) found that the perceived ethicality of possible solutions influences individual EDM, regardless of the moral intensity of that specific situation. Thus, it seems that content (i.e., the ethicality of solutions; one's role as actor, victim or bystander, etc.) influences EDM as well. In line with this, the results of the study described in this paper hint that the content of the critical incident is more important in the assessment of moral intensity than assumed until now. In future research this should be elaborated on.

A third and final conclusion that can be drawn from this study is that moral intensity is best captured with a more limited number of sub-dimensions than Jones (1991) originally proposed. This is in line with earlier findings that also hint at a different composition of moral intensity as a concept (Barnett, 2001; McMahon \& Harvey, 2006). Also, the general results regarding moral intensity indicate that the three dimensions revealed probability of harm social consensus and seriousness of harm score significantly higher in the high intensity condition than in the low intensity condition. Thus, servicemen seem to believe these elements of moral intensity are relevant in their assessment of overall moral intensity of critical incidents. It would be interesting to investigate whether these results are generalizable to other military organizations (i.e., in other cultures) and to other organizational cultures in general like other high-stake organizations such as 
a policing or health-care environment. These results give direction for future research regarding the dimensions of moral intensity.

\subsubsection{Limitations}

This study is not without limitations. First, we studied sensemaking in relation to moral intensity in a specific high-velocity environment, namely in military operations. Thus, it is still an open question whether these results can be generalized to other (non-hostile) environments. However, we believe the results give interesting insights for a broader organizational field, since in the extremis of the military operations, psychological processes are clearly demonstrated. Moreover, since we used a real-life sample of active-serving military personnel instead of the often-used student samples, we believe our results to be particularly reflective of sensemaking and moral intensity in 'real' environments. However, we suggest future research to look into ethical issues in different organizational settings, using a sample existing of 'true actors'.

Another limitation is related to the fact that this study was conducted within the Dutch military, a culture that can be seen as individualistic and as less sensitive to contextual variations than many other cultures (Giebels \& Taylor, 2009). Vitell, Nwachukwu and Barnes (1993) suggest that cultural dimensions are likely to influence ethical considerations (e.g., societal norms and principles) and as such ethical judgment and behavior. Thus, another suggestion for future research is to replicate our findings in different cultural contexts in order to gain insight in possible differences between cultural backgrounds.

A third limitation is related to the fact that we chose to ask the participants to evaluate the moral intensity of two different critical incidents. Although these incidents were considered different especially in terms of moral intensity and highly similar in other aspects, there may have been other features of the situations influencing the servicemen's reactions. Future research might want to focus on more controlled experimental studies with highly standardized scenario’s (e.g., May \& Pauli, 2002; Morris \& McDonald, 1995). Furthermore, we focused our inquiries on the early stages of EDM, where individuals assess the situation and try to make sense of it, instead of on the actual decision the participants would make. We assumed this to elicit less socially desirable answers as all sensemaking strategies may aid the process of responsible decisions and sensemaking is the stepping stone to such decisions. However, the proof of the pudding would be to examine the actual consequences of such internal deliberations.

Finally, and although we have employed an often used and theoretically sound instrument to measure moral intensity, there are other good measurements of moral intensity (e.g., Leitsch, 2004; May \& Pauli, 2002; Paolillo \& Vitell, 2002; Singhapakdi et al., 1996) which could be included in future research. We suggest for future research 
to focus on the further validation of the measurement of moral intensity. After all, even though the moral intensity scale displayed a Cronbach's alpha of .65 for all items, the components 'probability of harm' and 'social consensus' displayed relatively low alpha's. This suggests that moral intensity either composes of different dimensions than assumed or that the instrument we used does not fully capture the construct.

\subsubsection{Managerial Implications}

Brock et al. (2008) already argued that training in sensemaking improves EDM by means of the use of metacognitive reasoning strategies. These strategies are needed in order to objectively assess a complex dilemmatic situation. Due to the large impact their decisions may have, we recommend sensemaking training to become an integral part of military training. Also, individuals who have a clear understanding of the situation and the parties involved (including themselves) make more better-informed/meticulous decisions (cf. Brock et al., 2008; Ruedy \& Schweitzer, 2010).

Since the present study reveals that in high intensity situations certain 'higher order' tactics are less reported (i.e., analyzing and integrate available information; moral awareness and analyzing personal motivations) and having previous deployment experience influences the perception of moral intensity, the ethical dimensions of situations run the risk falling out of sight. Therefore, as they may not always be obvious, we plea for more attention for ethical challenges in pre-deployment training and in operational debriefings. Military units can learn collectively about ethical dimensions by sharing and reflecting on experienced ethical challenges. Moreover, next to sharing experiences, we believe additional training methods may be useful in order to simulate the circumstances of high intensity situations in such a manner that they appear realistic. Serious gaming and virtual reality (VR) can simulate the complexity and arousal in the mission area, going beyond theoretical/verbal sharing and 'knowing' towards true emotional and physical experiencing. As the cognitive deliberation process might be impaired by (high) intensity, training under similar conditions might be a valuable addition to current predeployment ethics programs.

Also, our results display that servicemen who have had earlier deployment experiences, score significantly lower on moral awareness (in both the high and low intensity condition). In the low intensity condition seeking outside help correlated negatively with having previous deployment experience. This might hint at a certain emotional numbing or moral blindness leading to overconfidence in own personal considerations (e.g., Verweij, 2005). For military leaders this is an important issue, for themselves to acknowledge as well as for their units. Especially in a context where experience often legitimizes choices, it is important to note that previous deployment experiences may lead to ethical issues being less salient, and as such influence an (accurate) assessment of the situation. 
In sum, we suggest to elaborate training in stimulating awareness of the ethical dimensions in a high-velocity context in different educational settings for all ranks regardless of their level of experience. After all, individuals who are not aware of the ethical dimensions in a situation will not start with EDM, and as such not with sensemaking in order to overcome self-serving judgments (Ruedy \& Schweitzer, 2010). When this is established and individuals are trained in using the different sensemaking strategies, teams can collectively learn from sharing and evaluating previous experiences. This can lead to better work outcomes in terms of moral attitudes and EDM behavior.

\subsubsection{Conclusion}

For the general EDM-research field, this study highlights the importance of including situational aspects. It offers some first insights into the impact of the moral intensity of a military critical incident on sensemaking processes. Our findings clearly show that the perceived moral intensity of the situation impacts the likelihood that a specific sensemaking tactic is employed. This is important information for leaders and managers who want to promote ethical behavior in the workplace, whether it is during military operations or during office duties. That is, the balanced combination of different sensemaking tactics may be necessary for an accurate perspective on the situation at hand for EDM, regardless of its' moral intensity.

In critical incidents the ethical challenges usually cannot be dealt with on the basis of standard procedures and daily routines. These situations are difficult to prepare for in the traditional sense of the warrior ethos (cf. Kilner, 2002). In training for highvelocity environments more attention could be placed on the importance of considering the broad range of sensemaking tactics available. Organizations that want to improve their employees' ability to make morally sound decisions, may also want to stimulate organizational learning (Stein \& Ahmad, 2009). This means that learning should focus on reviewing past actions and situations (Stein \& Ahmad, 2009) and implement these insights in the organization by transferring them by means of traditional as well as new educational methods, so that next generations are well versed for the ethical challenges they might encounter. However, it is important to keep in mind that our findings also suggest that having more military (deployment) experience does not necessarily lead to a more accurate perceived moral intensity assessment of the situation. 



\section{Chapter 6}

\section{General discussion}

Parts of this chapter are based on: De Graaff, M.C., De Vries, P.W., Van Bijlevelt, W.J. \& Giebels, E. (in press). Ethical leadership in the military, the gap between theory and practice in ethics education. In P.J. Olsthoorn (Ed.) Ethics in Military Leadership. Leiden: Brill.

We would like to express our gratitude to Desiree Verweij, Anne-Marie van Veldhuizen and Derek Suchard for their contributions to the parts of this chapter that were used for a peer-reviewed international book publication. 
A Dutch lieutenant is employed for a peacekeeping mission in an international team in Northern Africa during a major ebola outbreak. Some of her Dutch colleagues, members of her unit in the Netherlands, are scheduled to arrive at her international camp within a few days. A member of the coalition at the camp displays symptoms of the ebola virus. Therefore, nobody is allowed to leave the international camp. However, since the disease has not (yet) been officially diagnosed, units off base have not been informed about the possible outbreak in the camp. The commander does not want to alarm allied and opposing forces by calling attention to this premature conclusion regarding the situation in the camp. The Dutch lieutenant knows about the commander's decision, but when she speaks to her Dutch colleagues, she is torn: should she be loyal to her Dutch unit members and warn them about the possibility of an ebola outbreak in the camp, or should she be loyal to her Commander and not inform them? By doing the first, she may prevent her colleagues from contracting a possible ebola infection but she may also cause unnecessary commotion and compromise the camp; the latter would help maintain stability but may also expose her Dutch unit members to severe health risks...

(From an interview with a Dutch lieutenant regarding her deployment experiences.)

Ethical challenges, like the one described above, in all sorts and degrees are part of the daily business of uniformed professions, in particular the military (cf. Van Baarle, Bosch, Widdershoven, Verweij, \& Molewijk, 2015; Schut, De Graaff, Verweij, 2015; Richardson, Verweij, \& Winslow, 2004). Throughout this dissertation, I have used the term ethical challenge to refer to value-laden situations that are the result of interactions with other individuals, teams or cultures and make it difficult for the decision-maker to determine what the morally sound course of action is. As the stakes are usually high, the decisions servicemen arrive at may have far-reaching consequences. In ethical challenges like the one I began this chapter with, these tensions and stakes may be at the personal level (disciplinary measures because of insubordination), humanitarian and operational (the lives of fellow soldiers; isolation of the camp), or political (frictions between troops from different nations).

Individuals come to different decisions based on how they interpret the situation. Deliberate and intentional psychological mechanisms as well as unconscious and unintentional ones contribute to this process of interpretation when experiencing ethical challenges; I refer to that process as moral assessment throughout this dissertation (cf. De Graaff, Den Besten, Giebels \& Verweij, 2016a). The aim of this dissertation was 
to examine how moral assessment comes about in a military context. More particular, I wanted to distinguish the types of responses that result from encountering and recognizing ethical challenges. For this, I focused on the entire spectrum of reactions, namely on;

a) the 'dark side' of moral assessment that bias and cloud individual judgment negatively by disengaging from personal responsibility and behavior

b) the emotions that are evoked in ethical challenges, and that may amplify the processes in moral assessment

c) the positive 'sensemaking' strategies that help individuals understand the situation more completely.

I have used the theoretical models of Rest and Treviño as a starting point.

Firstly, in Rest's four component model (1986), after recognizing the moral dimensions of a situation, individuals engage in the next stage of what he labels ethical decision-making, in which they assess that specific situation. That is, individuals interpret the situation as it occurs and construct images of reality and probable consequences of actions that might be carried out in this situation. According to Rest, this assessment process allows individuals to form moral intentions that eventually lead to moral behavior. Rest refers to this process of assessing the ethical challenge as moral evaluation, which is quite similar to how I address this issue as moral assessment in this dissertation. Rest focuses on individual characteristics in (cognitive) moral development as the foundation for moral evaluation.

Where Rest (1986) focused on individual characteristics influencing moral judgment, Treviño (1986) pointed to the additional relevance of situational characteristics in ethical decision-making. Treviño argued that while an individual's moral development forms the basis for that person's response to a moral dilemma, it is insufficient to explain or predict ethical decision-making behavior (Treviño, 1986).

Based on these basic models, I began my empirical inquiry with a systematic review of previous research addressing moral assessment in the military over a period of almost three decades (1985-2012). I will give a detailed overview of the findings below, but the main conclusions that helped shape my orientation in the three studies examined were:

1) as in other decision-making domains, there is a growing interest in taking both cognitive and affective processes into account,

2) there is a knowledge gap in terms of the ethical challenges that servicemen themselves would put forward from their daily life experiences,

3) specific situational characteristics such as the type of ethical challenges or their moral intensity are barely addressed, and 
most studies focus on military officers (or officer cadets) as a research population ignoring other ranks. ${ }^{5}$

The main focus of the first study, presented in chapter 3, was to explore how individual cognitive characteristics of moral identity (i.e., the moral self, the sense of personal responsibility, and self-consistency) influenced expressions of moral disengagement. For this purpose, servicemen from all ranks were interviewed regarding ethical challenges they encountered during military operations. The second study, discussed in Chapter 4 and based on the same interview material, focused on the association between situational characteristics (i.e., the specific characteristics of the ethical challenge in terms of the type of interaction) with the emotions experienced and the response strategies preferred. In the third and final study, reported in Chapter 5, a large survey was conducted in which servicemen - again from different ranks and levels - were exposed to one of two scenarios. These scenarios differed in their level of moral intensity. The servicemen were asked to indicate how they would respond to the situation presented in the scenario, in terms of sensemaking tactics.

In this final chapter, Chapter 6, I will assess the merits of my doctoral studies. This chapter begins with a summary of the main findings from each chapter addressing the review study (Chapter 2) as well as the empirical studies (Chapters 3-5). Next, I will reflect on both the theoretical and practical implications of this dissertation. I will focus on commonalities and differences of the findings, what we can learn from these findings and which issues remain unanswered. Finally, I will identify the main strengths and weaknesses of this dissertation and provide suggestions and directions for future research.

\subsection{Summary of the Main Empirical Findings}

This dissertation explored the responses of military personnel to ethical challenges they encounter in daily practice as a result of interactions with other individuals, groups or cultures. In particular, the studies focused on moral assessment, one of the first stages of the moral judgment process. The purpose of this dissertation is to make both a theoretical as well as a practical contribution to the moral domain. Together with my fellow researchers, I conducted 4 studies. I conducted a review study regarding moral judgment research in the military. The results of the review study (presented in Chapter

\footnotetext{
${ }^{5}$ While that may appear to be a reasonable position in view of the fact that officers are in a leadership role and are often responsible for decisions that may have far reaching consequences, the increasing importance of the so-called 'strategic NCO' with the devolution of decision-making to lower ranks and the fact that ethical challenges are experienced regardless of rank makes investigating and stimulating the ethical fitness of all military personnel advisable if not essential.
} 
2) formed the foundation for the empirical studies that followed. These three empirical studies addressed how individuals respond to ethical challenges after having morally assessed the situation. Two of the empirical studies analyzed interview material focusing on moral identity and moral disengagement (Chapter 3), and emotions and response strategies in ethical challenges (Chapter 4). The third empirical study investigated the responses with regard to two prototypical ethical challenges by means of a survey.

The merit of this dissertation lies for one in the fact that these studies approach moral judgment, specifically moral assessment, empirically while theoretical notions without profound empirical analysis tend to dominate the field. The empirical studies described in this dissertation show how ethical challenges are part of everyday life of the soldiers when deployed abroad, regardless of rank. All service personnel were able to come up with morally challenging situations they were confronted with during deployment, varying in complexity as a consequence of role and rank. This dissertation identifies three main categories of ethical challenges:

A) Ethical challenges as a result of interactions with individuals with a different cultural background (e.g., personal norms vs. norms of the local population, for example regarding punishment of children)

B) Ethical challenges that are work related

a. and have to do with own troops, or one's own unit (e.g., loyalty towards fellow soldiers vs. obedience to ROE or staff orders)

b. ethical challenges as a result of cooperation with coalition partners with different norms and standards (e.g., personal norms vs. others' norms, for example regarding dealing with prisoners)

C) Ethical challenges resulting from interactions with the home front (e.g., questioning what personal experiences to share in order to protect relatives)

Fragments from interviews illustrating these types of ethical challenges are presented throughout the chapters of this dissertation. To our knowledge, this is the first time that day-to-day ethical issues related to military operations as experienced by all ranks - and not just officers - have been brought into view. These events run the entire gamut of ethical challenges from 'minor' dilemmas and frustrations to 'tragic incidents'. Research up to this point has devoted the lion's share of attention to local culture-related challenges, and challenges related to one's own troops such as struggles with mission goals and ROEs. The narratives of the servicemen show clearly, however, that there is much more to bring to the surface. As it turns out, morality is not only something for the armchair philosopher or the policy maker in The Hague; it is very much a relevant issue for the boots on the ground, too - troops, NCOs as well as officers. I therefore suggest elaborating further on these broad types of challenges in future research. 
Moreover, this dissertation advances the existing literature by exploring concepts related to moral assessment that have remained rather abstract up to this point. Firstly, the theoretical construct of moral identity as suggested by Blasi (1984), which refers to three components that together form an individual's state of being as a moral individual, was empirically investigated (Chapter 3). The results of the conducted empirical study suggest that an individual's moral self (i.e., the significance and salience of morality and values in an individual's considerations) and sense of personal responsibility are positively related to moral disengagement. This implies that a more prominent moral identity may lead to more utterances of moral disengagement. This is probably due to the fact that individuals want to maintain a positive image of themselves as moral agents. As study described in chapter 3 shows that the three components (i.e., moral self, sense of personal responsibility, and self-consistency) each have their own relevance in relation with moral disengagement, the study shows that it is relevant to distinguish the different elements in moral identity . An important next step will therefore be to further empirically elaborate how these components contribute to the entire process of ethical decision-making.

Secondly, the association between different types of ethical challenges and response strategies (i.e., moral disengagement, numbing and relativism) and the (mediating) effect of emotions in this relationship were addressed (Chapter 4). The works of Haidt (2003) on emotions formed one of the pillars for this investigation. Haidt (2003) introduced four families of moral emotions: other-condemning emotions, other-suffering emotions, other-praising emotions and self-conscious emotions. Most studies only focus on specific (families of) emotions (e.g., Rozin, Lowery, Imada, Haidt, 1999). This study however, explored the full range of emotions, from other-condemning emotions such as anger to other-praising emotions such as gratitude. Of the three response strategies relativism was the most reported response strategy. Moral justification, euphemistic labeling, displacement of responsibility (which are sub-categories within moral disengagement), relativism and numbing showed a strong positive correlation with local cultural and work-related ethical challenges. This means that when these types of challenges were reported more often, these specific response strategies were also reported more often. For the other types of ethical challenges and response strategies, such associations were not found. Other-condemning emotions (i.e., anger, contempt and disgust) proved to have a mediating effect on the relation between local cultural ethical challenges and relativism, and on the relation between moral justification and local cultural/work-related ethical challenges. So, the relationship between these response strategies and these specific types of ethical challenges is explained by the fact that other-condemning emotions are experienced. This study suggests that certain contexts evoke specific response strategies and that it is relevant to take notion of perceived emotions with regard to the ethical challenge as well. 
Thirdly, the situational aspects of the ethical challenge were examined with respect to moral intensity in relation to the use of sensemaking tactics. Jones's concept of moral intensity (Jones, 1991) is seldom addressed in military contexts, even though theory suggests that key features of the situation itself (such as its harmfulness) strongly influence the decision-maker. Prototypical situations from military deployment were presented to military personnel from all ranks, in order to explore this relationship. This study points out that the higher-order tactics analyzing personal motivations, analyzing and integrating available information and moral awareness, were used less frequently in high-intensity than in low intensity situations. In the high intensity situation, the different dimensions of moral intensity (i.e., seriousness of harm, probability of harm and social consensus) were not significant predictors for the use of certain sensemaking tactics. In the low intensity situation, however, it was found that the dimension seriousness of harm predicted moral awareness and seeking outside help to a marginal degree. The dimension probability of harm proved to be a significant predictor of the tactic recognizing the circumstances. Moreover, in line with previous research conducted outside the military context, this work shows that the moral intensity concept proposed by Jones consists of three rather than six dimensions (cf. Barnett, 2001; McMahon \& Harvey, 2006).

Together, the findings of the studies discussed in this dissertation address both affective and cognitive processes in moral judgment. One may conclude that the moral domain benefits from considering moral judgment through a lens that emphasizes both perspectives. In contrast with previous research that focused primarily on military leaders (officers and officer-cadets) as research population (see Chapter 2), military personnel from all ranks participated in all three of the empirical studies described in this dissertation. On the whole, leaders and subordinates score similarly when it comes to moral assessment. Only a few differences were found in specific elements between military personnel in a leadership position (officers and NCO's) and subordinates. Leaders score significantly higher than subordinates on all sub-categories of moral identity, i.e., moral self, sense of personal responsibility and self-consistency (i.e., an individual's personal reflection on the integration of moral standards and values in one's own daily life) (Chapter 3). Also, leaders display more other-praising emotions than their subordinates (Chapter 4). In low moral intensity situations, leaders score higher on the dimension of probability of harm and use the tactic of seeking outside help less often than their subordinates. In high moral intensity situations, leaders use the tactic of downgrading emotional overwhelming less often and analyzing and integrating available information more often. No differences were found between leaders and subordinates regarding response strategies (i.e., moral disengagement, relativism and numbing), low sense of moral worth and the number of ethical challenges they mentioned (Chapter $3 \& 4$ ). Moreover, being a leader did not influence the relationship between the use of 
sensemaking tactics and high or low moral intensity situations (Chapter 5). Due to the small sample size and the exploratory nature of this dissertation, these differences between leaders and subordinates should be interpreted cautiously. Nevertheless, they do suggest an interesting avenue for further research, as it may be concluded that morality is an issue worthy of being addressed empirically among all ranks.

A final remark and avenue for further research addresses an important issue with regard to dealing with ethical challenges. As these situations are quite delicate, especially in the military context where so much is at stake, it is important to remain sensitive to all parties, and to the interests and rights concerned. Be that as it may, some of the findings of the three empirical studies suggest that servicemen do not always show the degree of care and concern that these challenging circumstances call for. The results point out, for example, that critical self-reflection - showing awareness of personal biases and prejudice - is relatively underreported (Chapter 3 ) and higher-order sensemaking tactics are used less in high-intensity than in low-intensity situations (Chapter 5). This might be an important avenue for future exploration as the complexity of today's military operations, and consequently its ethical challenges, increases. As a consequence, this increasing complexity demands a higher degree of individual moral competence.

\subsection{Limitations and Future Research}

This dissertation argues that it is important to increase our knowledge of psychological mechanisms surrounding moral judgment. A critical reader might ask what these findings tell us about actual behavior of military personnel in morally challenging situations, however. After all, as in Rest's four component model, moral assessment is followed by moral intention and moral behavior (Rest, 1986). This critical reader has an important point. Although research usually shows that moral disengagement and unethical behavior are positively associated with each other (e.g., Hooks \& Mosher, 2005), we cannot rule out the possibility that explicit verbal expressions of moral disengagement are not necessarily associated with morally disengaging behavior. Similarly, when an individual does not express moral disengagement verbally, it does not mean that the actions that follow are all morally sound. That does not mean that the process of moral assessment itself is not worthy of study, however. After all, given the circumstances in which military personnel operate, it is often difficult if not impossible to distinguish right from wrong due to the ambiguous and uncertain nature of the ethical challenges. Moreover, this is amplified on many occasions by uncertainty about the likelihood of consequences when all courses of action include undesirable side effects (cf. Van den Berg \& Verweij, 2006). Hence, I consider it important to address the process underlying 
the actual course of action or decision. Although probably difficult to achieve, future research should try to include actual behavior.

Another limitation can be found in the use of 'true' narratives used in all three studies. Empirical interview material was a relevant underlying aspect of our research design in all the studies. In the studies the narratives of these interviews were used to make sure that the presented situations are deemed realistic and dilemmatic by the servicemen in the context of military operations. Nevertheless, interviews have the disadvantage that they depend on an individual's verbal capacity and reflexivity of the servicemen. This might have caused bias in our data. After all, individuals share what they want to share, but also only what they are capable of sharing. Due to our role in the organization and our military experience, we believe there was room for the participants to openly share their experiences with us. Their capability of sharing and transferring their experiences might be another issue, however. For example, research has shown that individuals differ in their competency of personal reflexivity (Caetano, 2015). A problem when interviewing individuals is that it is difficult to assess some sort of 'ground truth' (Baldwin, 1993). Especially when focusing on issues and experiences that are considered to be part of the 'grey-zone' (i.e., those situations of which individuals are uncertain about their personal accountability and the righteousness of their actions), it is very difficult to be sure about the accuracy of the servicemen's stories. As other recent studies conducted in European military organizations show similar ethical challenges (e.g., Schut \& Moelker, 2015; Van Baarle, Bosch, Widdershoven, Verweij \& Molewijk, 2015), we considered the material relevant for first explorations of individual moral assessment.

An avenue for further research is related to clinical PTSD research. Recent studies hint at the importance of moral issues in psychological/clinical problems, such as PTSD. In this dissertation, I have focused on aspects that have a strong tie with PTSD-related symptoms (sometimes in an ethical context referred to as moral injury; cf. Litz, Stein, Delaney, Lebowitz, Nash, Silva \& Maguen, 2009), like emotional numbing (Chapter 4) and moral intensity (Chapter 5). An interesting avenue for future research would be to look further into the relationship between moral assessment and individual wellbeing. For example, future work could examine moral injury as a result of exposure to ethical challenges. Also, I recommend addressing the idea that individuals make a distinction between moral personal and moral impersonal situations (Greene, Sommerville, Nystrom, Darley \& Cohen, 2001). That is, I presume that, due to awareness of personal roles and responsibility, situations in which an individual has a direct effect on the outcome of the situation cause more psychological distress than situations in which an individual only indirectly influences the outcome. For example, when an NCO is asked for advice regarding a certain decision the platoon commander needs to make, the NCO will probably feel less distress in case of negative consequences than in the situation in which 
it is 'his call'. Moreover, a difficult situation presents itself when servicemen are willing to act in a morally sound way, but are confronted with moral transgressions of other parties, such as opposing forces. This might lead to high psychological distress and subsequent moral transgressions from the troops themselves, as it is likely that such situations evoke anger, and other-condemning emotions related to moral disengagement (Chapter 4). Thus, it would be interesting to address the different types of ethical challenges more profoundly in future research.

Another issue that is important to address in future research is the influence of group dynamic processes on the decision-making process. In my studies, I have addressed individual moral assessment. It may be that group dynamics influence the assessment itself as well as the eventual decision made. In addition to group dynamics, selfcategorization might play a role as well. The core of theories of social identity and selfcategorization is that, for various substantive and sometimes superficial reasons, a group of people start seeing themselves as an in-group, by comparison with a salient outgroup. It is to be expected that in such a situation, phenomena such as in-group favoritism and outgroup devaluation, which have been well documented in psychological research, will rear their rather ugly heads, and influence assessment and decision even when objective consideration suggests another course of action. The very fact that in groups and out groups have been defined would arguably lead to treating outgroup members differently than in-group members. Moreover, threats to one's group and group identity may spark aggression and revenge towards the out group (Fischer, Haslam, \& Smith, 2010).

\subsection{Practical Implications}

It is not uncommon for military personnel to question whether the moral domain is worth considering. Some feel that it is a field of interest for those who are not operational or on active duty within the military. Some servicemen regard moral considerations and ethics as something for the armchair moralist, but not at all useful in the daily tasks of military operations (cf. De Graaff \& Van den Berg, 2010). The studies in this dissertation reveal clearly, however, that ethical challenges are omnipresent in the real world of military practice at all levels and in all kinds of situations, and are likely to evoke responses as well. Yet identifying psychological mechanisms that are relevant to the moral judgment process may be of little use if military personnel consider these insights as irrelevant to their daily practice. After all, when people fail to see the value of these insights - and consequently fail to transfer them into practice (or training) immoral behavior may still occur and situations may escalate as they did in the Abu Ghraib incident. I therefore emphasize the importance of ethics education in order to stimulate and preserve moral competence in military personnel. 


\subsubsection{Individual Implications}

Although the Dutch military acknowledges the importance of the concept of moral competence, it is being put in to practice in the organization only to a very small degree (De Graaff, \& Van den Berg, 2010). In officer-training programs, moral competence is trained by requiring officers to reflect upon the elements of moral competence described in Chapter 1 in order to become 'moral professionals'. Moral professionalism covers (1) awareness of one's own personal values and the values of others; (2) the ability to identify the moral dimensions within situations; (3) the ability to come to a meticulous judgment; (4) the ability to communicate this judgment; (5) the willingness and ability to act accordingly; and (6) the willingness and ability to be held accountable and feel responsible for one's personal actions in the situation (cf. De Graaff \& Van den Berg, 2010; Verweij, 2007; Wortel \& Bosch, 2011). The students formulate their personal learning objectives in this respect (for example, to suspend immediate judgment, or to listen without prejudice and bias). NCO training and other ranks education, on the other hand, focus more on 'real soldier activities' (e.g., basic weapon skills and drills) and normative ethics such as behavioral codes; only NCOs who are trained ethics instructors receive a 9-days training in moral competence (Van Baarle et al., 2015). However, even when having participated in ethics education most servicemen still seem to find it difficult to recognize the moral dimensions in events as they are unfolding. For example, I described in Chapter 5 how almost half of the dilemma situations that were described as true events by military personnel were not considered to include moral dimensions by fellow servicemen. One explanation may be that, in most ethics-training sessions, the dilemmas are presented in a manner that does little to encourage participants to take the perspective of the individuals being portrayed. The scenarios are presented as hypothetical, theoretical, or rather extreme, or are conveyed in text or film, all of which may create a distance between the subject matter and the participants' own world or experiences. In only a few instances are servicemen invited to share their own dilemmas. I believe that this causes servicemen to grow accustomed to recognizing dilemma situations and moral dimensions when explicitly asked to do so (for example when reflecting upon a movie like Armadillo in the classroom), but not in daily-life situations. Similarly, in dilemma-training sessions in which participants are encouraged to share personal experiences, most of them (even those in the social domain, such as coaching and HR) seem to have difficulty to come up with a dilemma. In a civilian educational context similar difficulties have been acknowledged by Walker and colleagues (Walker, Pitts, Hennig \& Matsuba, 1995). They argued that this problem occurs among individuals who attach no significance to their moral identity in daily life (Walker, 2004). Thus, it seems that military personnel are better able to recognize moral dimensions in life-and-death challenges presented to them as scenarios (the so-called 
tragic dilemmas), than in more prevalent yet less obvious morally challenging situations. I suggest integrating ethics education, including the psychological mechanisms, into military operational exercises. For this educational purpose, the examples of ethical challenges described throughout this dissertation can be used to inspire and instigate collective and individual learning. At present, evaluation of operational exercises mainly focuses on how the skills and drills were carried out and procedures followed. I believe that it is relevant to address the ethical dimensions in debriefings as well. It is only then that servicemen learn to recognize the full scope of ethical challenges in their profession, as well as the processes that inhibit them or their teams from doing 'the right thing'.

Critical reflection and bildung. Recent studies have suggested how mindfulness, i.e., having an open mind in difficult situations, may contribute to ethical decision-making. Research appears to show that a state of mindfulness facilitates individuals' awareness of the ethical dimensions in their context (e.g., Ruedy, \& Schweitzer, 2010; Shapiro, Jazaieiri, \& Goldin, 2012). In addition, critical reflection may help to incorporate the process of making choices in dilemma situations and an individual's deliberation about the ethical challenges into the moral self-concept. In consequence, reflexivity and openness may contribute to moral awareness and restrain moral disengagement. Investing in the servicemen's reflexivity may help to prevent moral stress and promote better moral judgment.

I therefore suggest that military training be amended to embrace both reflexivity and mindfulness as psychological competencies in actual training sessions. Enabling servicemen to let go of their fixed frameworks and checklists when necessary may well cause them to have an open mind in experiencing the situation without immediate biased interpretation. Feedback from superiors and team members can help in developing these competencies by making them more salient in discussions.

\subsubsection{Organizational Implications}

This dissertation shows that responses are not only a result of a certain level of moral development, but are also heavily affected by the situational circumstances. Predeployment training can benefit from the insights my work has produced regarding the influence of the type of situation and moral intensity on response strategies and sensemaking. For example, knowing that team-related and local-culture related ethical challenges in particular have a strong influence on moral assessment - and consequently on moral disengagement - it then becomes important for military leaders to act appropriately within their units when they encounter such a challenge in the area of operations. These insights are quite practical and can be of use for military leaders in preparing their units adequately. It provides the servicemen with the opportunity to 
think about such ethical challenges in order to prepare them mentally for the possibility of encountering such a situation.

Moreover, after deployment, the Dutch military aims to record lessons identified and learned from the deployment. These lessons tend to focus on procedures and operations and not so much on the ethical dimensions of the mission. It is therefore possible that important ethical issues are not recognized and, hence, not transferred in the preparation of follow-up deployments. For example, within the Afghanistan deployments, this proved to be an issue in relation with encountering so-called Bacha $B a z i^{6}$. Since there were no guidelines or indicators available, individual Dutch troops in each subsequent deployment were presented with situations that to them were new and unexpected, although many of their predecessors had encountered them as well. Apparently, such experiences - like encountering Bacha Bazi - were not shared with those who would likely benefit most from them, i.e., the servicemen brought in to relieve their colleagues when their deployment was up. This in fact required everyone to reinvent the wheel repeatedly (cf. Schut, \& Van Baarle, 2014). In terms of the procedures and operations as well as the ethical dimension of the mission, the experiences of one's predecessors in operational environments provide highly relevant information on which to base attempts for improvement.

Compliance versus integrity culture. Within many organizational contexts, a shift from a compliance-based (or rule-oriented) culture towards an integrity culture (Verhezen, 2010) can be noticed. This shift towards attention for the 'thinking soldier' and 'moral professionalism' is also present in the armed forces of the Netherlands (cf. De Graaff \& Van den Berg, 2010). In such an integrity culture, organizations rely on the moral competence of individual employees to come to morally sound decisions. The insights - such as the low sense of moral worth and strong feelings of personal responsibility towards one's own unit (Chapter 3), the influence of emotions on moral assessment (Chapter 4) and the diminished critical thinking in high intensity situations (Chapter 5) - indicate that concentrating only on individual competence gives rise to irresponsible behavior. After all, the circumstances during deployment may instigate group-dynamics and other psychological mechanisms that blur individual decision-making, which, in

\footnotetext{
${ }^{6}$ A 2010 documentary brought to light that adult Afghan men had sexual relationships with preadolescent boys (Bacha Bazi) who were dressed up as women and danced for their masters (Schut, \& Van Baarle, 2014). Dutch military leaders (e.g., platoon commanders) were in some cases offered the services of these boys by their hosts (e.g., high-ranking Afghan police officers) resulting in very delicate situations; Dutch military leaders were required to strike a balance between the taboo in the Afghan culture regarding this tradition, the high positions of their hosts offering Bacha Bazi services and the necessity of maintaining good working relationships with these individuals, and personal opinions and Western norms regarding basic human rights and human dignity (cf. Schut, \& Van Baarle, 2014).
} 
the worst case, may lead to moral transgressions such as in the Abu Ghraib case (cf. Bartone, 2010; Zimbardo, 2007). I therefore suggest that compliance and rules are relevant, but only to a limited extent. With regard to the 'grey zone', it is important for individuals to be morally competent so that they can weigh and decide what to do in order to make morally sound decisions (cf. Verhezen, 2010). In this grey zone, adequate rules and procedures may help to overcome the pitfall of the psychological mechanisms just described; research has taught us, however, that rules or codes of conduct simplify reality and that too many rules do not have the desired effect and may even instigate incidents (Kaptein \& Schwartz, 2008). It is therefore necessary to balance between moral competence and compliance. I think we should not be forced to choose between them, but should integrate both perspectives in order to prevent the occurrence of incidents in the high-stakes environment of the military.

\subsection{Final Remarks}

Profound lessons from military operations in the past few decades have taught us that moral dimensions cannot be removed from the operational field (De Graaff \& Van den Berg, 2010). It would be unrealistic to believe that military personnel do not encounter challenging situations in moral terms when they are conducting military operations. Thus, moral competence is not only desired; it is mandatory. Gaining insight in how first interpretations of difficult situations come to existence by an individual's moral assessment is therefore vital. The most important conclusion of this dissertation is that ethical challenges are omnipresent on a day-to-day basis for all military ranks, and that both individual and situational characteristics influence individual moral assessment. It is not as simple as 'switching' to the moral high ground: this dissertation shows that moral assessment depends on more than just an on/off switch. The studies described in this dissertation form another step towards understanding the ethical decision-making process in complex environments. I hope it instigates new (managerial/organizational and educational) developments to prepare the armed forces for dealing with the ethical challenges they face in today's military operations. 



\section{References}


Alexander, D. A., \& Klein, S. (2001). Ambulance personnel and critical incidents. Impact of accident and emergency work on mental health and emotional well-being. The British Journal of Psychiatry, 178, 76-81.

Andres, M., \& Rietveld, N. (2012), It's not over till it's over. In R. Beeres, J. van der Meulen, J. Soeters, A. Vogelaar (Eds.), Mission Uruzgan Collaborating in multiple coalitions for Afghanistan (pp 295-308). Amsterdam: Pallas Publications.

Aquino, K., Reed, A., Thau, S., \& Freeman, D. (2007). A grotesque and dark beauty: How moral identity and mechanisms of moral disengagement influence cognitive and emotional reactions to war. Journal of Experimental Social Psychology, 43, 385-392.

Aquino, K., \& Reed II, A. (2002). The self-importance of moral identity. Journal of Personality and Social Psychology, 83, 1423-1440.

Bagozzi, R.P., Sekerka, L.E., \& Hill, V. (2009). Hierarchical motive structures and their role in moral choices. Journal of Business Ethics, 90, 461-486.

Bagozzi, R. P., Sekerka, L. E., Hill, V., \& Sguera, F. (2013). The role of moral values in instigating morally responsible decisions. The Journal of Applied Behavioral Science, 49, 69-94.

Baker, D. P. (2012). Making good better: A proposal for teaching ethics at the service academies. Journal of Military Ethics, 11, 208-222.

Baldwin, J. (1993). Police interview techniques: Establishing Truth or Proof?. British Journal of Criminology, 33(3), 325-352.

Bandura, A. (1986). Social foundations of thought and action: A social cognitive theory. Englewood Cliffs, NJ: Prentice-Hall, Inc.

Bandura, A. (1991), Social cognitive theory of moral thought and action. In W. M. Kurtines \& J.L.Gewitz (Eds.), Handbook of Moral Behaviour and Development (pp. 45-103). Hillsdale, NJ: Erlbaum.

Bandura, A. (1999). Moral disengagement in the perpetration of inhumanities. Personality and Social Psychology Review, 3, 193-209.

Bandura, A. (2002). Selective moral disengagement in the exercise of moral agency. Journal of Moral Education, 31, 101-119.

Bandura, A., Barbaranelli, C., Caprara, G. V., \& Pastorelli, C. (1996). Mechanisms of moral disengagement in the exercise of moral agency. Journal of Personality and Social Psychology, 71, 364-374.

Baron, R. M., \& Kenny, D. A. (1986). The moderator-mediator variable distinction in social psychological research: Conceptual, strategic, and statistical considerations. Journal of Personality and Social Psychology, 51, 1173-1182.

Bartone, P. T. (2010). Preventing prisoner abuse: Leadership lessons of Abu Ghraib. Ethics \& Behavior, 20, 161-173.

Barnett, T. (2001). Dimensions of moral intensity and ethical decision making: An empirical study. Journal of Applied Social Psychology, 31, 1038-1057.

Benham Rennick, J. (2012). Canadian values and military operations in the twenty-first century. Armed Forces \& Society, 39, 511-530.

Biernacki, P., \& Waldorf, D. (1981). Snowball sampling: Problems and techniques of chain referral sampling. Sociological Methods \& Research, 10, 141-163.

Blais, A. R., \& Thompson, M. M. (2008). Decision processes in military moral dilemmas: The role of moral intensity and moral judgment (No. DRDC-TORONTO-TR-2008-190). Toronto, Canada: Defence Research and Development.

Blais, A.R., \& Thompson, M. M. (2013). What Would I Do? Civilians' Ethical Decision Making in Response to Military Dilemmas. Ethics \& Behavior, 23, 237-249.

Blasi, A. (1983). Moral cognition and moral action: A theoretical perspective. Developmental Review, 3, 178-210.

Blasi, A., \& Glodis, K. (1995). The development of identity. A critical analysis from the perspective of the self as subject. Developmental Review, 15, 404-433. 
Blumer, H. (1969). Symbolic interactionism: Perspective and method. Englewood Cliffs, N.J.: Prentice Hall.

Brock, M. E., Vert, A., Kligyte, V., Waples, E. P., Sevier, S. T., \& Mumford, M. D. (2008). Mental models: An alternative evaluation of a sensemaking approach to ethics instruction. Science and engineering ethics, 14, 449-472.

Broesder, W. A., den Buijs, T. P. O., Vogelaar, A. L., \& Euwema, M. C. (2015). Can soldiers combine swords and ploughshares? The construction of the warrior-peacekeeper role identity survey (WPRIS). Armed Forces \& Society, 41, 519-540.

Burnell, K.J., Boyce, N., \& Hunt, N. (2011). A good war? Exploring British veterans' moral evaluation of deployment. Journal of Anxiety Disorders, 25, 36-42.

Caetano, A. (2015). Defining personal reflexivity A critical reading of Archer's approach. European Journal of Social Theory, 18, 60-75.

Caughron, J. J., Antes, A. L., Stenmark, C. K., Thiel, C. E., Wang, X., \& Mumford, M. D. (2011). Sensemaking strategies for ethical decision making. Ethics \& Behavior, 21, 351-366.

Christensen, S. L., \& Kohls, J. (2003). Ethical decision making in times of organizational crisis. A framework for analysis. Business \& Society, 42, 328-358.

Connor-Smith, J. K., \& Flachsbart, C. (2007). Relations between personality and coping: A metaanalysis. Journal of Personality and Social Psychology, 93, 1080-1107.

Cook, M. L., \& Syse, H. (2010). What should we mean by 'Military Ethics'? Journal of Military Ethics, 9, 119-122.

Corbin, J. M., \& Strauss, A. (1990). Grounded theory research: Procedures, canons, and evaluative criteria. Qualitative Sociology, 13, 3-21.

Crittenden, K. S., \& Hill, R. J. (1971). Coding reliability and validity of interview data. American Sociological Review, 36, 1073-1080.

Cummings, M. L., \& Nehme, C. E. (2010). Modeling the impact of workload in network centric supervisory control settings. In S. Kornguth, R. Steinberg, \& M. D. Matthews (Eds.), Neurocognitive and Physiological Factors during High-tempo Operations (pp. 23-40). Surrey, UK: Ashgate.

Cunha, M.P., Rego, A., \& Clegg, S.R. (2010). Obedience and evil: From Milgram and Kampuchea to normal organizations. Journal of Business Ethics, 97, 291-309.

De Graaff, M. C., F.D.A. Den Besten, E. Giebels, D.E.M. Verweij (2016a). Moral judgement in war and peacekeeping operations: An empirical review. In T.R. Elßner, \& R. Janke (Eds.), Didactics of Military Ethics: From Theory to Practice (pp. 75-111). Leiden: Brill.

De Graaff, M.C., Schut, M., Verweij, D.E.M., Vermetten, E., \& Giebels, E. (2016b). Emotional reactions and moral judgment: The effects of morally challenging interactions in military operations. Ethics \& Behavior, 26, 14-31.

De Graaff, M.C., \& Van den Berg, C.E.M. (2010), Moral Professionalism within the Royal Netherlands Armed Forces. In J. Stouffer \& S. Seiler (Eds.), Military Ethics, International Perspectives (pp 1-24). Kingston: Canadian Defence Academy Press.

De Graaff, M. C., \& Van Gils, M. J. (2012), Military Professionalism, an organizational challenge by itself. In J. Stouffer \& D. Lindsay (Eds.), Threats to Military Professionalism, International Perspectives (pp. 57-70). Kingston: Canadian Defence Academy Press.

De Graaff, M.C., De Vries, P.W., Van Bijlevelt, W.J., \& Giebels, E. (forthcoming). Ethical leadership in the military, the gap between theory and practice. In P.J. Olsthoorn (Ed.) Ethics in Military Leadership. Leiden: Brill.

Desplaces, D. E., Melchar, D. E., Beauvais, L. L., \& Bosco, S. M. (2007). The impact of business education on moral judgment competence: An empirical study. Journal of Business Ethics, 74, 73-87.

Detert, J. R., Treviño, L. K., \& Sweitzer, V. L. (2008). Moral disengagement in ethical decision making: a study of antecedents and outcomes. Journal of Applied Psychology, 93, 374.

Eisenhardt, K. M. (1989). Making fast strategic decisions in high-velocity environments. Academy of Management Journal, 32, 543-576. 
Elm, D. R., \& Radin, T. J. (2012). Ethical decision making: Special or no different? Journal of Business Ethics, 107, 313-329.

Emmons, R. A., \& Crumpler, C. A. (2000). Gratitude as a human strength: Appraising the evidence. Journal of Social and Clinical Psychology, 19, 56-69.

Engelhard, I. M., Olatunji, B. O., \& de Jong, P. J. (2011). Disgust and the development of posttraumatic stress among soldiers deployed to Afghanistan. Journal of Anxiety Disorders, $25,58-63$.

Eriksen, J.W. (2010), Should soldiers think before they shoot? Journal of Military Ethics, 9, 195218.

Erikson, E.H. (1946). Ego development and historical change; clinical notes. In A. Freud, H. Hartmann \& E. Kris (Eds.), The Psychoanalytic Study of the Child, Vol. 2, pp. 359-396. New York: International Universities Press.

Fischer, P., Haslam, S. A., \& Smith, L. (2010). "If you wrong us, shall we not revenge?" Social identity salience moderates support for retaliation in response to collective threat. Group Dynamics, 14(2), 143-150.

Folger, R., Cropanzano, R., \& Goldman, B. (2005). What is the relationship between justice and morality? In J. Greenberg \& J.A. Coquitt (Eds.), Handbook of Organizational Justice, (pp. 215-245). London: Lawrence Erlbaum Associates, Publishers.

Frankel, M. S. (1989), Professional codes: Why, how, and with what impact? Journal of Business Ethics, 8, 109-115.

Frey, B. F. (2000a). The impact of moral intensity on decision making in a business context. Journal of Business Ethics, 26, 181-195.

Frey, B. F. (2000b). Investigating moral intensity with the world-wide web: A look at participant reactions and a comparison of methods. Behavior Research Methods, Instruments, \& Computers, 32, 423-431.

Friedman, M. (2006). Posttraumatic stress disorder among military returnees from Afghanistan and Iraq. American Journal of Psychiatry, 163, 586-593.

Fry, S.T., Harvey, R.M., Hurley, A.C., \& Foley, B.J. (2002), Development of a model of moral distress in military nursing. Nursing Ethics, 9, 373-387.

Fumagalli, M., Ferrucci, R., Mameli, F., Marceglia, S., Mrakic-Sposta, S., Zago, S., . . Pravettoni, G. (2010). Gender-related differences in moral judgments. Cognitive Processing, 11, 219-226.

Gable, S. L., \& Gosnell, C. L. (2013). Approach and avoidance behavior in interpersonal relationships. Emotion Review, 5, 269-274.

Gibbs, J. C., Arnold, K. D., \& Burkhart, J. E. (1984). Sex differences in the expression of moral judgment. Child Development, 55, 1040-1043.

Giebels, E., \& Taylor, P. J. (2009). Interaction patterns in crisis negotiations: persuasive arguments and cultural differences. Journal of Applied Psychology, 94, 5.

Gire, J.T., \& Williams, T.D. (2007), Dissonance and the honor system: Extending the severity of threat phenomenon. The Journal of Social Psychology, 147, 501-509.

Glaser, B. G., \& Strauss, A. L. (2009). The discovery of grounded theory: Strategies for qualitative research. New Brunswick: Transaction Books.

Gouveia, W. A. (2004). An analysis of moral dissent: An army officer's public protest of the Vietnam war. Journal of Military Ethics, 3, 53-60.

Grassiani, E. (2012). When soldiers explain. Discursive strategies used by Israeli conscripts when recounting their experiences in the field. In: M.M. Six-Hohenbalken \& N. Weiss (Eds.), Violence Expressed: an Anthropological Approach, pp. 91-108. Farnham (UK): Ashgate Publishing, Ltd.

Greene, J., \& Haidt, J. (2002). How (and where) does moral judgment work? Trends in Cognitive Sciences, 6, 517-523.

Greene, J. D., Sommerville, R. B., Nystrom, L. E., Darley, J. M., \& Cohen, J. D. (2001). An fMRI investigation of emotional engagement in moral judgment. Science, 293(5537), 2105-2108. 
Gross, J. J., \& Levenson, R. W. (1993). Emotional suppression: physiology, self-report, and expressive behavior. Journal of Personality and Social Psychology, 64, 970-987.

Haidt, J. (2001). The emotional dog and its rational tail: a social intuitionist approach to moral judgment. Psychological Review, 108, 814-834.

Haidt, J. (2003). The moral emotions. In R. J. Davidson, K. R. Scherer \& H. H. Goldsmith (Eds.), Handbook of affective sciences (pp. 852-870). Oxford: Oxford University Press.

Haidt, J., Graham, J., \& Joseph, C. (2009). Above and below left-right: Ideological narratives and moral foundations. Psychological Inquiry, 20, 110-116.

Hannah, S. T., \& Avolio, B. J. (2010), Moral potency: Building the capacity for character-based leadership. Consulting Psychology Journal: Practice and Research, 62, 291-310.

Hannah, S.T., Avolio, B.J., \& Walumbwa, F.O. (2011), Relationships between authentic leadership, moral courage, and ethical and pro-social behaviors. Business Ethics Quarterly, 21, 555-578.

Hannah, S. T., Schaubroeck, J. M., Peng, A. C., Lord, R. G., Treviño, L. K., Kozlowski, S. W., .. . Doty, J. (2013). Joint influences of individual and work unit abusive supervision on ethical intentions and behaviors: A moderated mediation model. Journal of Applied Psychology, 98(4), 579-592.

Harkrider, L. N., Tamborski, M. A., Wang, X., Brown, R. P., Mumford, M. D., Connelly, S., \& Devenport, L. D. (2013). Threats to Moral Identity: Testing the Effects of Incentives and Consequences of One's Actions on Moral Cleansing. Ethics \& Behavior, 23, 133-147.

Harris, N. (2003). Reassessing the dimensionality of the moral emotions. British Journal of Psychology, 94, 457-473.

Hayibor, S., \& Wasieleski, D. M. (2009). Effects of the use of the availability heuristic on ethical decision-making in organizations. Journal of Business Ethics, 84, 151-165.

Higgins, E. T., Bond, R. N., Klein, R., \& Strauman, T. (1986). Self-discrepancies and emotional vulnerability: how magnitude, accessibility, and type of discrepancy influence affect. Journal of Personality and Social Psychology, 51, 5-15.

Hitlin, S. (2007). Doing good, feeling good: Values and the self's moral center. The Journal of Positive Psychology, 2, 249-259

Hofstede, G. H. (2001). Culture's Consequences: Comparing Values, Behaviors, Institutions and Organizations across Nations. Thousand Oaks: Sage.

Hutcherson, C. A., \& Gross, J. J. (2011). The moral emotions: A social-functionalist account of anger, disgust, and contempt. Journal of Personality and Social Psychology, 100, 719-737.

Janhunen, K. (2012). A comparison of Likert-type rating and visually-aided rating in a simple moral judgment experiment. Quality \& Quantity, 46, 1471-1477.

Janoff-Bulman, R. (2004). Posttraumatic growth: Three explanatory models. Psychological Inquiry, 15, 30-34.

Jackson, D. N., Wroblewski, V. R., \& Ashton, M. C. (2000). The impact of faking on employment tests: Does forced choice offer a solution? Human Performance, 13, 371-388.

Jeffrey, T.B., Rankin, R.J., \& Jeffrey, L.K. (1992). In service of two masters: The ethical-legal dilemma faced by military psychologists. Professional Psychology: Research and Practice, 23, 91-95.

Jones, T. M. (1991). Ethical decision making by individuals in organizations: An issue-contingent model. Academy of Management Review, 16, 366-395.

Kahane, G., \& Shackel, N. (2010). Methodological issues in the neuroscience of moral judgement. Mind \& Language, 25, 561-582.

Kahneman, D., \& Sunstein, C. R. (2005). Cognitive psychology of moral intuitions. In J.P. Changeus, A. R. Damasio, W. Singer, \& Y. Christen (Eds.), Neurobiology of Human Values, (pp. 91-105). Berlin: Springer.

Kaptein, M., \& Schwartz, M.S. (2008). The effectiveness of business codes: a critical examination of existing studies and the development of an integrated research model. Journal of Business Ethics, 77, 11-127. 
Karssing, E. D. (2001). Morele Competentie in Organisaties. Assen: Koninklijke van Gorcum.

Killgore, W.D.S., Killgore, D.B., Day, L.M., Li, C., Kamimori, G.H., \& Balkin, T.J. (2007). The effects of 53 hours of sleep deprivation on moral judgment. Sleep, 30, 345-352.

Kilner, P. (2002). Military leaders' obligation to justify killing in war. Military Review, 82, 24.

Knickmeyer, E. (2006, May 27). In Haditha, Memories of a Massacre. The Washington Post. Retrieved: October 7, 2014, from http://www.washingtonpost.com/wp-dyn/content/ article/2006/05/26/AR2006052602069.html

Kohlberg, L. (1981). Essays on Moral Development. Vol. 1, The Philosophy of Moral Development: Moral Stages and the Idea of Justice. San Fransisco, CA: Harper \& Row.

Kopala, B., \& Burkhart, L. (2005). Ethical dilemma and moral distress: proposed new NANDA diagnoses. International Journal of Nursing Terminologies and Classifications, 16, 3-13.

Kramer, E. H. (2007). Organizing doubt: grounded theory, army units and dealing with dynamic complexity (Vol. 22). Copenhagen: Business School Press.

Kramer, E. H., van Bezooijen, B., \& Delahaij, R. (2010). Sensemaking during operations and incidents. In J. Soeters, P.C. van Fenema, R. Beeres, (Eds.), Managing Military Organizations: Theory and Practice, (pp. 126-137). New York: Routledge.

Kroll, J., \& Egan, E. (2004). Psychiatry, moral worry, and the moral emotions. Journal of Psychiatric Practice, 10, 352-360.

Landis, J. R., \& Koch, G. G. (1977). The measurement of observer agreement for categorical data. Biometrics, 33, 159-174.

Landgren, J. (2005). Supporting fire crew sensemaking enroute to incidents. International Journal of Emergency Management, 2, 176-188.

Leitsch, D. L. (2004). Differences in the perceptions of moral intensity in the moral decision process: An empirical examination of accounting students. Journal of Business Ethics, 53, 313-323.

Lerner, J. S., \& Keltner, D. (2001). Fear, anger, and risk. Journal of Personality and Social Psychology, 81, 146-159.

Liebes, T., \& Blum-Kulka, S. (1994). Managing a moral dilemma: Isreali soldiers in the intifada. Armed Forces \& Society, 21, 45-68.

Lifton, R. J. (1973). The sense of immortality: On death and the continuity of life. American Journal of Psychoanalysis, 33, 3-15.

Limbert, C. (2004). Psychological well-being and job satisfaction amongst military personnel on unaccompanied tours: The impact of perceived social support and coping strategies. Military Psychology, 16, 37-51.

Lincoln, S. H., \& Holmes, E. K. (2010). The psychology of making ethical decisions: What affects the decision? Psychological Services, 7, 57-64.

Linn, R. (1988), Moral judgment in extreme social contexts: Soldiers who refuse to fight and physicians who strike? Journal of Applied Social Psychology, 18, 1149-1170.

Linn, R. (1989), Hypothetical and actual moral reasoning of Israeli selective conscientious objectors during the war in Lebanon. Journal of Applied Developmental Psychology, 10, 1936.

Linn, R. (2002), Soldiers with conscience never die. They are just ignored by their society. Moral disobedience in the Israel defense forces. Journal of Military Ethics, 1, 57-76.

Litz, B. T. (1992). Emotional numbing in combat-related post-traumatic stress disorder: A critical review and reformulation. Clinical Psychology Review, 12, 417-432.

Litz, B. T., Schlenger, W. E., Weathers, F. W., Caddell, J. M., Fairbank, J. A., \& LaVange, L. M. (1997). Predictors of emotional numbing in posttraumatic stress disorder. Journal of Traumatic Stress, 10, 607-618.

Litz, B. T., Stein, N., Delaney, E., Lebowitz, L., Nash, W. P., Silva, C., \& Maguen, S. (2009). Moral injury and moral repair in war veterans: A preliminary model and intervention strategy. Clinical Psychology Review, 29, 695-706. 
Martin, L. E., Stenmark, C. K., Thiel, C. E., Antes, A. L., Mumford, M. D., Connelly, S., \& Devenport, L. D. (2011). The influence of temporal orientation and affective frame on use of ethical decision-making strategies. Ethics \& Behavior, 21, 127-146.

May, D.R., \& Pauli, K.P. (2002). The Role of Moral Intensity in Ethical Decision Making: A Review and Investigation of Moral Recognition, Evaluation, and Intention. Business \& Society, 41, 84-117.

McCullough, M. E., Kilpatrick, S. D., Emmons, R. A., \& Larson, D. B. (2001). Is gratitude a moral affect? Psychological Bulletin, 127, 249-266.

McMahon, J.M, \& Harvey, R.J. (2006). An Analysis of the Factor Structure of Jones' Moral Intensity Construct. Journal of Business Ethics, 64, 381-404.

McMahon, J. M., \& Harvey, R. J. (2007). The effect of moral intensity on ethical judgment. Journal of Business Ethics, 72, 335-357.

Milgram, N. A., Orenstein, R., \& Zafrir, E. (1989). Stressors, personal resources, and social supports in military performance during wartime. Military Psychology, 1, 185-199.

Ministerie van Defensie (2015). Kerngegevens 2015, Feiten en Cijfers. Den Haag: Ministerie van Defensie.

Moens, A. (2003). Lessons for peacekeepers: Srebrenica and the NIOD report. Canadian Foreign Policy Journal, 10, 141-158.

Morris, S. A., \& McDonald, R. A. (1995). The role of moral intensity in moral judgments: An empirical investigation. Journal of Business Ethics, 14, 715-726.

Moelker, R. (2013), The Genesis of the "Dutch Approach" to Asymmetric Conflicts Operations in Uruzgan and the "Softly, Softly" Manner of Approaching the Taleban. Armed Forces \& Society, 40, 96-117.

Morris, S. A., \& McDonald, R. A. (1995). The role of moral intensity in moral judgments: An empirical investigation. Journal of Business Ethics, 14, 715-726.

Mumford, M.D., Connelly, S., Brown, R.P., Murphy, S.T., Hill, J.H., Antes, A.L., et al. (2008). A sensemaking approach to ethics training for scientists: Preliminary evidence of training effectiveness. Ethics \& Behavior, 18, 315-339.

Nijenhuis, E. R., \& Van der Hart, O. (2011). Dissociation in trauma: a new definition and comparison with previous formulations. Journal of Trauma \& Dissociation, 12, 416-445.

Nilsson, S., Sjöberg, M., Kallenberg, K., \& Larsson, G. (2011). Moral stress in international humanitarian aid and rescue operations: A grounded theory study. Ethics \& Behavior, 21, 49-68.

Noy, C. (2008). Sampling knowledge: The hermeneutics of snowball sampling in qualitative research. International Journal of Social Research Methodology, 11, 327-344.

Nucci, L. P., \& Turiel, E. (1978). Social interactions and the development of social concepts in preschool children. Child Development, 49, 400-407.

O'Fallon, M. J., \& Butterfield, K. D. (2005). A review of the empirical ethical decision-making literature: 1996-2003. Journal of Business Ethics, 59, 375-413.

Offstein, E. H., \& Dufresne, R. L. (2007). Building strong ethics and promoting positive character development: The influence of HRM at the United States Military Academy at West Point. Human Resource Management, 46, 95-114.

O’Kane, A., Fawcett, D., \& Blackburn, R. (1996), Psychopathy and moral reasoning: Comparison of two classifications. Personality and Individual Differences, 20, 505-514.

Oliver, K. (2003). Atrocity, Authenticity and American Exceptionalism:(Ir) rationalising the Massacre at My Lai. Journal of American Studies, 37, 247-268.

Oliver, D., \& Roos, J. (2003). Dealing with the unexpected: Critical incidents in the LEGO Mindstorms team. Human Relations, 56, 1057-1082.

Olsen, O.K., Eid, J., \& Johnsen, B.H. (2006). Moral behavior and transformational leadership in Norwegian naval cadets. Military Psychology, 18, 37-56.

Olsen, O.K., Pallesen, S., \& Eid, J. (2010). The impact of partial sleep deprivation on moral reasoning in military officers. Sleep, 33, 1086-1090. 
Olsthoorn, P. (2005). Honor as a motive for making sacrifices. Journal of Military Ethics, 4 183197.

Olsthoorn, P., Bollen, M. \& Beeres, R. (2013). Dual loyalties in military medical care - between ethics and effectiveness. In H. Amersfoort, R. Moelker, J. Soeters \& D. Verweij (Eds.), Moral Responsibility and Military Effectiveness, pp. 79-96. Den Haag: Asser Press.

Olsthoorn, P., Meijer, M., \& Verweij, D. (2010). Managing moral professionalism in military operations. In J. Soeters, P. C. V. Fenema \& R.Beeres (Eds.), Managing Military Organizations: Theory and Practice (pp. 138-149). New York: Routledge.

Paine, L. S. (1994), Managing for organizational integrity. Harvard Business Review, 72, 106-117.

Paine, L. S. (1996), Moral thinking in management: An essential capability. Business Ethics Quarterly, 6, 477-492.

Park, N., \& Peterson, C. (2006). Moral competence and character strengths among adolescents: The development and validation of the values in action inventory of strengths for youth. Journal of adolescence, 29, 891-909.

Paolillo, J. G., \& Vitell, S. J. (2002). An empirical investigation of the influence of selected personal, organizational and moral intensity factors on ethical decision making. Journal of Business Ethics, 35, 65-74.

Perez, C. (2012). The soldier as lethal warrior and cooperative political agent: on the soldier's ethical and political obligations toward the indigenous other. Armed Forces \& Society, 38, 177-204.

Pfister, A., De Graaff, M. C., \& Van Gils, M. J. (2012), The dynamic-five-factor model as a compass for military professionalism: a comparison between Switzerland and the Netherlands. In J. Stouffer \& D. Lindsay (Eds.), Threats to Military Professionalism, International Perspectives, pp. 195-215. Kingston: Canadian Defence Academy Press.

Pizarro, D. (2000). Nothing more than feelings? The role of emotions in moral judgment. Journal for the Theory of Social Behaviour, 30, 355-375.

Podsakoff, P. M., \& Organ, D. W. (1986), Self-reports in organizational research: Problems and prospects. Journal of Management, 12, 531-544.

Preacher, K. J., \& Hayes, A. F. (2008). Asymptotic and resampling strategies for assessing and comparing indirect effects in multiple mediator models. Behavior Research Methods, 40, 879891.

Rademaker, A. R., Vermetten, E., \& Kleber, R. J. (2009). Multimodal exposure-based group treatment for peacekeepers with PTSD: A preliminary evaluation. Military Psychology, 21, 482-496.

Reger, M.A., Etherage, J.R., Reger, G.M., \& Gahm, G.A. (2008). Civilian psychologists in an army culture: The ethical challenge of cultural competence. Military Psychology, 20, 21-35.

Reichberg, G., \& Syse, H. (2002). Humanitarian Intervention: A Case of Offensive Force? Security Dialogue, 33, 309-322.

Reimer, K. S., DeWitt Goudelock, B. M., \& Walker, L. J. (2009). Developing conceptions of moral maturity: Traits and identity in adolescent personality. The Journal of Positive Psychology, 4, 372-388.

Rest, J. R. (1986). Moral development: Advances in research and theory. Praeger Publishers.

Rest, J. R. \& Barnett, R. (1986). Moral Development: Advances in Theory and Research. New York: Praeger.

Reynolds, S. J., \& Ceranic, T. L. (2007). The effects of moral judgment and moral identity on moral behavior: an empirical examination of the moral individual. Journal of Applied Psychology, 92, 1610-1624.

Richardson, R., \& Kramer, E. H. (2006). Abduction as the type of inference that characterizes the development of a grounded theory. Qualitative Research, 6, 497-513.

Richardson, R., Verweij, D., \& Winslow, D. (2004). Moral fitness for peace operations. Journal of Political and Military Sociology, 32, 99-113. 
Robinson, P. (2007). Ethics training and development in the military. Parameters, 37, 23-36.

Roeser, S. (2006). The role of emotions in judging the moral acceptability of risks. Safety Science, 44, 689-700.

Roeser, S. (2010). Intuitions, emotions and gut reactions in directions about risks: toward a different interpretation of 'neuroethics'. Journal of Risk Research, 13, 175-190.

Rozin, P., Lowery, L., Imada, S., \& Haidt, J. (1999). The CAD triad hypothesis: a mapping between three moral emotions (contempt, anger, disgust) and three moral codes (community, autonomy, divinity). Journal of Personality and Social Psychology, 76, 574.

Ruedy, N. E., \& Schweitzer, M. E. (2010). In the moment: The effect of mindfulness on ethical decision making. Journal of Business Ethics, 95, 73-87.

Saxena, V., \& Babu, N. (2013). Expanding the domain of morality by going beyond moral reasoning: emerging trends in moral research. Psychological Studies, 58, 326-334.

Schaubroeck, J. M., Hannah, S. T., Avolio, B. J., Kozlowski, S. W. J., Lord, R. G., Treviño, L. K., Dimotakis, N., \& Peng, A. C. (2012), Embedding ethical leadership within and across organization levels. Academy of Management Journal, 55, 1053-1078.

Schön, D. A. (1991). The reflective practitioner, how professionals think in action. London: Ashgate.

Schut, M., de Graaff, M. C., \& Verweij, D. (2015). Moral emotions during military deployments of Dutch forces A qualitative study on moral emotions in intercultural interactions. Armed Forces \& Society, 41, 616-638.

Schut, M., \& Moelker, R. (2015). Respectful agents: between the Scylla and Charybdis of cultural and moral incapacity. Journal of Military Ethics, 14, 232-246.

Schut, M., \& Van Baarle, E. (2014) Dansjongens in de schijnwerpers, een artikel over de praktijk van bacha bazi. Carre, 2, 32-37.

Schwarz, N. (1999), Self-reports: how the questions shape the answers. American Psychologist, 54, 93-105.

Schwartz, H., \& Jacobs, J. (1979). Qualitative sociology - a method to the madness. New York: Free Press.

Sekerka, L.E., Bagozzi, R.P., \& Charnigo, R. (2009). Facing ethical challenges in the workplace: Conceptualizing and measuring professional moral courage. Journal of Business Ethics, 89, 565-579.

Seiler, S., Fischer, A., \& Ooi, Y. P. (2010). An interactional dual-process model of moral decision making to guide military training. Military Psychology, 22, 490-509.

Seiler, S., Fischer, A., \& Voegtli, S. A. (2011). Developing moral decision-making competence: A quasi-experimental intervention study in the Swiss armed forces. Ethics \& Behavior, 21, 452-470.

Shapiro, S. L., Jazaieri, H., \& Goldin, P. R. (2012). Mindfulness-based stress reduction effects on moral reasoning and decision making. The Journal of Positive Psychology, 7(6), 504-515.

Simmons, C. A., \& Rycraft, J. R. (2010). Ethical challenges of military social workers serving in a combat zone. Social Work, 55, 9-18.

Singh, J. J., Vitell, S. J., Al-Khatib, J., \& Clark III, I. (2007). The role of moral intensity and personal moral philosophies in the ethical decision making of marketers: A cross-cultural comparison of China and the United States. Journal of International Marketing, 15, 86-112.

Singhapakdi, A., S. J. Vitell \& K. L. Kraft. (1996). Moral intensity and ethical decision-making of marketing professionals, Journal of Business Research, 36, 245-255.

Soeters, J., (2004), Geweld en conflict, het ontstaan en verloop van burgeroorlogen, etnische strijd en terrorisme. Amsterdam: Boom.

Sonenshein, S. (2007). The role of construction, intuition, and justification in responding to ethical issues at work: The sensemaking-intuition model. Academy of Management Review, $32,1022-1040$.

Sparks, J. R., \& Siemens, J. C. (2014). Judgment difficulty and the moral intensity of unethical acts: A cognitive response analysis of dual process ethical judgment formation. Ethics \& Behavior, 24, 151-163. 
Strauss, A., \& Corbin, J. (1994). Grounded theory methodology. An overview. In: Denzin \& Lincoln (Eds.), Handbook of Qualitative Research, (pp. 273-285). Thousand Oaks, CA: Sage Publications Inc.

Stein, E. W., \& Ahmad, N. (2009). Using the analytical hierarchy process (AHP) to construct a measure of the magnitude of consequences component of moral intensity. Journal of Business Ethics, 89, 391-407.

Steinbauer, R., Renn, R. W., Taylor, R. R., \& Njoroge, P. K. (2014). Ethical leadership and followers' moral judgment: The role of followers' perceived accountability and self-leadership. Journal of Business Ethics, 120, 381-392.

Tangney, J. P., Stuewig, J., \& Mashek, D. J. (2007). Moral emotions and moral behavior. Annual Review of Psychology, 58, 345.

Thiel, C. E., Connelly, S., \& Griffith, J. A. (2011). The Influence of Anger on Ethical Decision Making: Comparison of a Primary and Secondary Appraisal. Ethics \& Behavior, 21, 380403.

Thiel, C. E., Bagdasarov, Z., Harkrider, L., Johnson, J. F., \& Mumford, M. D. (2012). Leader ethical decision-making in organizations: Strategies for sensemaking. Journal of Business Ethics, 107, 49-64.

Thoma, S. J., Rest, J. R., \& Davison, M. L. (1991). Describing and testing a moderator of the moral judgment and action relationship. Journal of Personality and Social Psychology, 61, 659-669.

Thompson, M. M., \& Jetly, R. (2014). Battlefield ethics training: integrating ethical scenarios in high-intensity military field exercises. European Journal of Psychotraumatology, 5.

Thompson, M. M., Thompson, M. H., \& Adams, B. D. (2008). Moral and Ethical Dilemmas in Canadian Forces Military Operation: Qualitative and Descriptive Analyses of Commanders' Operational Experiences (No. DRDC-TORONTO-TR-2008-183). Toronto, Canada: Defence Research and Development.

Tillman, C. J., Hood, A. C., Lawrence, E. R., \& Kacmar, K. M. (2015). When birds of a feather flock together: The role of core-self evaluations and moral intensity in the relationship between network unethicality and unethical choice. Ethics \& Behavior, 25, 458-481.

Treviño, L. K. (1986). Ethical decision making in organizations: A person-situation interactionist model. Academy of Management Review, 11, 601-617.

Tripodi, P. (2006). Peacekeepers, moral autonomy and the use of force. Journal of Military Ethics, $5,214-232$.

Van Baarda, T.A., \& Verweij, D.E.M. (2006). Military Ethics: Its Nature and Pedagogy. In: T.A. van Baarda \& D.E.M. Verweij, Military Ethics the Dutch Approach: a Practical Guide. (pp. 1-24) Leiden: Brill/Martinus Nijhoff Publishers.

Van Baarle, E., Bosch, J., Widdershoven, G., Verweij, D., \& Molewijk, B. (2015). Moral dilemmas in a military context. A case study of a train the trainer course on military ethics. Journal of Moral Education, 44(4), 457-478.

Van den Berg, C., \& Soeters, J. (2009). Self-perceptions of soldiers under threat: A field study of the influence of death threat on soldiers. Military Psychology, 21, S16-S30.

Van Den Berg, C. E., \& Verweij, D. E. M. (2006). Ethics in the Royal Netherlands Army: The Development of Morally Competent Military Personnel, from Recruit to Veteran. In T.A. van Baarda \& D.E.M. Verweij, Military Ethics the Dutch Approach: a Practical Guide, (pp. 117-134). Leiden: Brill/Martinus Nijhoff Publishers.

Visser, M. (2003). Gregory Bateson on deutero-learning and double bind: A brief conceptual history. Journal of the History of the Behavioral Sciences, 39, 269-278.

Verhezen, P. (2010). Giving voice in a culture of silence. From a culture of compliance to a culture of integrity. Journal of Business Ethics, 96(2), 187-206.

Verweij, D., (2007). Morele professionaliteit in de militaire praktijk. In: J. Kole, \& D. de Ruyter, (eds.), Werkzame idealen - ethische reflecties op professionaliteit. (pp. 126-138) Assen: Koninklijke van Gorcum. 
Verweij, D.E.M. (2013), Responsibility as the 'Ability to Respond' Adequately. In H. Amersfoort, R. Moelker, \& J. Soeters (Eds.), Moral Responsibility \& Military Effectiveness, pp. 11-32. Den Haag: Asser Press.

Verweij, D., Hofhuis, K., \& Soeters, J. (2007). Moral judgement within the armed forces. Journal of Military Ethics, 6, 19-40.

Vitell, S. J., Nwachukwu, S. L., \& Barnes, J. H. (1993). The effects of culture on ethical decisionmaking: An application of Hofstede's typology. Journal of Business Ethics, 12, 753-760.

Walker, L. J. (2004). Gus in the gap: Bridging the judgment-action gap in moral functioning. In D.K. Lapsley \& D. Narvaez (Eds.), Moral Development, Self, and Identity, pp. 1-20. Mahwah: Lawrence Erlbaum Associates Inc., Publishers.

Walker, D., \& Myrick, F. (2006). Grounded theory: an exploration of process and procedure. Qualitative Health Research, 16, 547-559.

Warner, C.H., Appenzeller, G.N., Mobbs, A., Parker, J.R., Warner, C.M., Grieger, T., \& Hoge, C.W. (2011), Effectiveness of battlefield-ethics training during combat deployment: A program assessment. Lancet, 378, 915-924.

Wead, S. (2015). Ethics, Combat, and a Soldier's Decision to Kill. Military Review, 95, 69.

Whetham, D. (2008). The challenge of ethical relativism in a coalition environment. Journal of Military Ethics, 7, 302-316.

Weber, J., \& Wasieleski, D. (2001). Investigating Influences on Managers' Moral Reasoning. The Impact of Context and Personal and Organizational Factors. Business \& Society, 40, 79-110.

Weick, K. E. (1993). The collapse of sensemaking in organizations: The Mann Gulch disaster. Administrative Science Quarterly, 38, 628-652.

Weick, K. E. (1995). Sensemaking in Organizations. Thousand Oaks, CA: Sage.

Weick, K. E., Sutcliffe, K. M., \& Obstfeld, D. (2005). Organizing and the process of sensemaking. Organization Science, 16, 409-421.

Weibull, L. (2012). Emotion matters. Emotion management in Swedish Peace Support Operations (Doctoral Dissertation). Karlstad: University Studies.

Whetham, D. (2008). The challenge of ethical relativism in a coalition environment. Journal of Military Ethics, 7, 302-316.

White, R.D. (1999). Are woman more ethical? Recent findings on the effects of gender upon moral development. Journal of Public Administration Research and Theory, 9, 459-471.

Williams, K.R. (2010). An assessment of moral and character education in initial entry training (IET). Journal of Military Ethics, 9, 41-56.

Wortel, E., \& Bosch, J. (2011). Strengthening moral competence: A 'train the trainer' course on military ethics. Journal of Military Ethics, 10, 17-35.

You, D., Maeda, Y., \& Bebeau, M. J. (2011). Gender differences in moral sensitivity: A metaanalysis. Ethics \& Behavior, 21, 263-282.

Yu, Y.M. (2013). Between an Example and a Precept, which has Greater Importance? A Comparison of the Channels of Socialization in Military Ethics. Ethics \& Behavior, 23, 341359.

Zimbardo, P. (2007). The Lucifer effect: Understanding how good people turn evil. New York: ME Sharpe. 
Summary in English 
This dissertation increases our knowledge on ethical decision-making (EDM) in terms of moral assessment by examining how military professionals interpret ethically challenging situations, addressing both deliberate and unintentional psychological mechanisms that contribute to this process, the responses that are associated with moral assessment, and the relationships between them. In short, the following overarching, central question formed the foundation of this dissertation:

\section{How do military professionals make moral assessments of day-to-day ethical challenges during military deployment and how does this process relate to individual responses to those ethical challenges?}

To answer this central question four sub-questions that define the focus of each study more specifically have been formulated. In each empirical study, different variables of interest that came forward as issues of interest from the review study are addressed. The review study is presented in Chapter 2 .

\section{Systematic Review of Moral Judgment Research within the Military}

What aspects of moral judgment, and moral assessment in particular, have been studied previously in a military context, and what avenues for further exploration follow this systemic review?

In Chapter 2, a review study is described providing an overview of current empirical research conducted within a military context regarding moral judgment. A sensitive search of online databases was conducted and 33 relevant empirical studies published in ISI journals between 1985 and 2013 were identified. The systematic review revealed conceptual, methodological and contextual issues. Regarding the conceptual issues, the analysis of these 33 publications shows that the majority of the studies used a cognitive rather than an affective or integrative (combined) approach to moral judgment. As is the case with moral judgment research in other disciplines, the affective and the integrative approach are both relatively new and are increasingly receiving scientific attention. Moreover, within the cognitive approach, there has been almost no study of situational characteristics - i.e., specific features of the ethical issue itself such as moral intensity (for an exception, see Seiler, Fischer \& Voegtli, 2011). Regarding the methodology used in these studies, the review shows that most research uses interviews or surveys about hypothetical situations to question military officers (e.g., Olsen, Pallesen \& Eid, 2010) and/or includes case studies (e.g., Reger, Etherage, Reger \& Gahm, 2008). The true events that have been studied, concentrated primarily on incidents (such as Abu Ghraib, e.g., Bartone, 2010) focusing on moral transgressions rather than on ethical challenges themselves. Lower-ranking servicemen, those who actually find themselves in combat 
situations and the operations themselves, are systematically overlooked in studies of moral judgment processes in the military. Furthermore, studies conducted in the U.S. military tend to dominate the field. Since over a period of almost 30 years, a modest amount of only 33 empirical studies could be identified, and taken together with the other insights, this study highlights the fact that further empirical research in this field is required.

\section{Moral Identity in Moral Judgment}

How do military personnel construct their moral identity in relation to the ethical challenges of military operations, and how does this relate to verbalized moral disengagement?

In Chapter 3, a theoretical framework was introduced regarding moral identity and moral disengagement. Although moral character has been prominent in moral judgment literature, there has been almost no empirical study of moral identity (cf. Walker, 2004). We used Blasi's (1983) self-model of moral functioning, as a starting point.

The model integrates moral cognition and moral personality in order to explain moral behavior (cf. Walker, 2004). It consists of three sub-categories: 1) the moral self, 2) the individual's sense of personal responsibility, and 3) self-consistency (Blasi, 1983; Walker, 2004). Blasi argues that one's moral identity is strongly intertwined with selective disengagement processes since individuals are prone to maintain their perceived self-integrity (Walker, 2004). This psychological mechanism of withdrawing from one's personal standards of what is morally acceptable is referred to as moral disengagement (Bandura, 1999).

We explored the construction of the servicemen's moral identity in relation to ethical challenges encountered in military operations. We also explored the relationship between moral identity and verbalized moral disengagement. Semi-structured interviews with 45 servicemen were content-coded and analyzed. The results point at three patterns. The first pattern shows that a higher ethical awareness of moral challenges in the situation (i.e., a stronger moral identity and a higher awareness of ethical challenges) evokes more justifications for one's own behavior; this pattern was observed regardless of rank.

The second pattern we found suggests that leaders (officers as well as NCOs) display more proneness towards moral identity than their followers. This is reflected by significantly higher scores on all elements of moral identity. This can be explained by the fact that they receive more extensive ethics education than their subordinates. Also, the leaders' sense of proactive personal responsibility is significantly higher, which arguably follows from the duties connected to their role. Moreover, leaders score higher on selfconsistency. 
The third pattern showed that critical self-reflection and self-assessment (measured in self-consistency) are relatively underreported even though moral identity is quite prominent in the servicemen regardless of their rank. These results may hint at the strong urge of individuals to maintain a positive self-image (Kaufman, 1974): When individuals are well aware of the ethical dimensions of the situations they find themselves in, they may also be more sensitive to their own roles in those situations. So, when their actions run counter their personal beliefs of being a 'good person' they tend to use response strategies like moral disengagement in order to maintain their sense of 'good self'. That could point to a possible drawback of fostering a strong moral identity.

\section{Emotional Reactions in Moral Judgment}

What different types of ethical challenges are military personnel confronted with on a dayto-day basis during deployment, and how do these types relate to perceived emotions and the response strategies moral disengagement, numbing and relativism?

The study described in Chapter $\mathbf{4}$ explored the association between different types of morally challenging situations during military deployment and response strategies (e.g., moral justification), and how they are fueled by emotions. To develop our theoretical framework, we integrated insights from research on moral emotions (e.g., Haidt, 2001), moral numbing (e.g., Lifton, 1973), ethical relativism (e.g., Whetham, 2008) and moral disengagement (e.g., Bandura, 1999). In this study, the same 45 narratives that were used in chapter 3 were content-coded and analyzed in terms of the type of interaction, the perceived emotions (i.e., other-condemning emotions, other-suffering emotions, other-praising emotions, self-conscious emotions) and responses (i.e., relativism, moral disengagement and numbing). Team-related challenges were described most, followed by local-cultural challenges. In terms of their responses, the participating servicemen indicated they experienced other-condemning emotions following a moral challenge most often and reported using relativism most often.

Moral justification, relativism and numbing showed a strong correlation with local cultural and work-related ethical challenges. Other-condemning emotions (i.e., anger, contempt and disgust) proved to have a mediating effect on the relationship between local cultural ethical challenges and relativism, and on the relation between moral justification and local culture/work-related ethical challenges. This study suggests that the contexts influence the specific response strategies that are evoked. The results also show that it is relevant to take notion of emotions, in particular other condemning emotions, as they shape military reactions to occurring ethical challenges. 


\section{Sensemaking Tactics in Moral Judgment}

How is the moral intensity of an ethical challenge related to the likelihood of employment of certain sensemaking tactics in military professionals during military operations?

In the third and final empirical study, reported in Chapter 5, we considered moral assessment from a unique integrative perspective: that is, we addressed specifically how individuals make sense of ethical challenges that differ in moral intensity, such as the harmfulness (to a larger group of people), the likeliness to occur, and the direct impact on or personal relevance for the decision-maker (Jones, 1991). For this, we followed the person-situation interactionist approach suggested by Treviño (1986) by exploring the sensemaking tactics in the moral assessment of a situation. The tactics that we used encompass four broad categories that are in line with existing research in this field: emotion regulation, forecasting, self-reflection, and information integration (cf. Brock et al., 2008; Thiel, Bagdasarov, Harkrider, Johnson \& Mumford, 2012). Emotion regulation refers to the collection of tactics that deal with downgrading emotional overwhelming. Forecasting refers to the extent wherein individuals anticipate the consequences of different alternate actions. Self-reflection is the category that encompasses tactics that show active awareness of personal biases and moral reflection upon the situation at hand. The final category information integration refers to tactics that help individuals to collect and analyze available information such as seeking outside help.

Two prototypical ethical challenges (one low and one high in moral intensity) from military deployment - drawn from interview material of earlier studies - were selected and presented to 325 active duty military personnel in a questionnaire. The results show that less attention goes out to higher level critical thinking in the decision making process when the intensity is high. Also, those who had experienced more than one deployment, seemed to exhibit a certain degree of numbing as those servicemen consider the situations as less intense as their less experienced colleagues. These results seem to suggest that although servicemen need to give room for thorough consideration and deliberation when considering ethical challenges, this 'capacity' can be further enhanced. The findings described in this chapter add to those of chapter 3 pointing out the relevance of increased attention for individual reflexivity and critical thinking in situations that require such competencies.

\section{Ethical Challenges}

Upon completion of the empirical studies, we were able to make a categorization of morally challenging situations. As ethical challenges always involve an intrapersonal "clash" of values caused by an interaction with others, we categorized the ethical challenges described by the servicemen according to the type of 'others' involved in the situation. We could identify three broad types of morally challenging interactions: 
A. Ethical challenges as a result of interactions with individuals with a different cultural background (e.g., personal norms vs. norms of the local population, for example regarding punishment of children)

B. Ethical challenges that are work related

a. and have to do with one's own troops, or unit (e.g., loyalty towards fellow soldiers vs. obedience to ROE or staff orders)

b. or result from cooperation with coalition partners with different norms and standards (e.g., personal norms vs. others' norms, for example regarding dealing with prisoners)

C. Ethical challenges resulting from interactions with the home front (e.g., doubting what personal experiences to share in order to protect relatives)

\section{Final remarks}

To conclude, profound lessons from military operations in the past few decades have taught us that moral dimensions cannot be removed from the operational field (De Graaff \& Van den Berg, 2010). It would be unrealistic to believe that military personnel do not encounter situations that are challenging in moral terms when they are involved in military operations where they find themselves in continuous social interactions with individuals from other cultural backgrounds, the home front and fellow servicemen. Moral competence is therefore not only desirable: it is mandatory. Consequently, gaining insight in how the initial interpretations of difficult situations come into being during an individual's moral assessment is vital. The most important conclusions of this dissertation are that ethical challenges are present on a day-to-day basis for all military ranks, and that both individual and situational characteristics influence individual moral assessment. It is not as simple as 'taking' the moral high ground: this dissertation shows that moral assessment depends on more than just an on/off switch. It demands developing and nurturing individual moral competence in order for military personnel to learn to adequately deal with situations that they are not prepared for and where interpretations are influenced by psychological mechanisms such as moral disengagement, emotions or perceived moral intensity. I consider it an organizational duty for the military leadership to facilitate its personnel by noting lessons from previous deployments and to support servicemen both during deployment and afterwards when they are processing the often intense and sometimes tragic ethical challenges. Taken together, the studies described in this dissertation are one more step on the path to understanding the ethical decisionmaking process in complex environments. 

Nederlandse samenvatting 
Hoe militairen morele uitdagingen interpreteren en welke reacties samenhangen met dit proces van morele beoordeling (moral assessment), was het onderwerp van de vier studies beschreven in deze dissertatie. Morele uitdagingen zijn situaties voortkomend uit sociale interacties waarin de beslisser met een conflict van waarden of belangen wordt geconfronteerd, dat niet op basis van routine of vastgestelde procedures kan worden opgelost. De onderstaande overkoepelende onderzoeksvraag vormde de basis voor deze dissertatie.

\section{Hoe maken militairen een morele beoordeling van dagelijkse morele uitdagingen die zij zelf ervaren gedurende militaire operaties, en hoe is dit proces gerelateerd aan individuele reacties op deze morele uitdagingen?}

\section{Systematische review}

Welke aspecten van morele oordeelsvorming, en specifiek van morele beoordeling, zijn bestudeerd in de militaire context en welke richtingen voor verder onderzoek volgen uit deze systematische review?

Hoofdstuk 2 beschrijft een overzicht en analyse van empirisch onderzoek dat is gepubliceerd in ISI journals tussen 1985-2013 naar morele oordeelsvorming in de militaire context. Uit de analyse kwamen 33 publicaties naar voren die aan de inclusiecriteria voldeden. Deze studies zijn meegenomen in de systematische review. De meeste onderzoeken bleken een cognitieve benadering te hebben. De affectieve benadering waarin emoties en intuïtie een rol spelen in morele oordeelsvorming bleef onderbelicht. De geïntegreerde benadering, waarin zowel cognitie als subjectieve beleving (ook wel affect) een rol spelen, werd het minst ingezet. Dit is in lijn met onderzoek naar moreel oordeelsvermogen in andere contexten. Daarnaast bleek uit de analyse dat binnen de cognitieve benadering weinig aandacht is uitgegaan naar het bestuderen van situationele kenmerken die van invloed zijn op de oordeelsvorming, zoals morele intensiteit.

De review liet zien dat in de meeste gevallen gebruik werd gemaakt van hypothetische situaties die in interviews en vragenlijsten aan militaire officieren werden voorgelegd. 'Echte gebeurtenissen' zijn minder onderzocht. Bovendien zijn dit dan vaak studies gericht op het onderzoeken van incidenten waarbij gekeken werd naar morele overschrijdingen en niet zozeer naar de morele uitdagingen zelf, zoals de Abu Ghraib gevangenis-incidenten in Irak (Bartone, 2010). Daarbij kwam naar voren dat juist militairen in lagere rangen, de zogenaamde boots on the ground in weinig onderzoeken zijn meegenomen; de aandacht gaat vooral uit naar militaire leiders. Bovendien blijkt dat studies uitgevoerd in de Verenigde Staten het onderzoeksveld domineren. De analyse en het feit dat er over een periode van 30 jaar slechts 33 empirische studies konden worden gevonden, geven de relevantie en richting voor empirisch onderzoek in dit veld aan. 


\section{Morele identiteit in morele oordeelsvorming}

Op welke wijze construeren militairen hun morele identiteit in relatie met de ethische vraagstukken van militaire operaties, en hoe is dit gelinkt aan uitingen van morele zelfafmelding?

In Hoofdstuk 3 is een theoretisch raamwerk voor morele identiteit en morele zelfafmelding (moral disengagement) geïntroduceerd. Ook al gaat er in de literatuur veel aandacht uit naar de zogenaamde 'morele karakterontwikkeling', er is nauwelijks empirische onderzoeksmateriaal over morele identiteit beschikbaar (vgl. Walker, 2004). In deze empirische studie gebruikten we het self-model of moral functioning van Blasi (1983) als startpunt. Het model integreert cognitie en persoonlijkheid om moreel gedrag te verklaren. Het model bestaat uit 3 elementen: 1) het morele 'zelf', 2) het gevoel van persoonlijke verantwoordelijkheid van een individu, en 3) zelf-consistentie. Blasi beargumenteert dat de morele identiteit sterk verweven is met wat Bandura (1999) noemt morele zelf-afmelding.

Wij onderzochten de constructie van de morele identiteit van militairen in relatie tot de ethische vraagstukken die zij in de militaire operatie tegenkomen. Daarnaast exploreerden wij de relatie tussen morele identiteit en verbale morele zelf-afmelding. Hiervoor zijn 45 semigestructureerde interviews getranscribeerd en geanalyseerd. De resultaten duiden op drie patronen. Het eerste patroon laat zien dat een sterker moreel besef (d.w.z. een sterkere morele identiteit en een sterker bewustzijn van ethische dimensies in situaties) meer 'vergoelijking' van het eigen gedrag oproept. Dit patroon werd gevonden in alle rangen.

Het tweede patroon toonde dat kritische zelfreflectie en zelf-assessment (gemeten in zelf-consistentie) ondergerapporteerd was, ook wanneer de morele identiteit verder duidelijk naar voren komt. Deze resultaten lijken te wijzen op een sterke behoefte van individuen om een positief zelfbeeld te behouden (vgl. Kaufman, 1974). Wanneer individuen zich bewust zijn van de ethische dimensies in een situatie waarin zij zich bevinden, dan zijn ze zich ook meer bewust van hun eigen rol in deze situaties. Dus, wanneer hun handelen tegen hun persoonlijke overtuigingen en mensbeeld van een 'goed persoon' indruist zijn zij geneigd om respons-strategieën als morele zelf-afmelding te gebruiken om een positief zelfbeeld te behouden.

Het derde patroon dat we hebben gevonden suggereert dat militaire leiders (zowel officieren als onderofficieren) een sterkere morele identiteit hebben dan hun ondergeschikten. Leiders scoren hoger op de elementen van morele identiteit dan hun volgers. Ditkanverklaardwordendoordatleidersmeerethiekonderwijsendilemmatraining krijgen dan hun ondergeschikten. Daarnaast is het verantwoordelijkheidsgevoel van leiders significant hoger dan dat van hun ondergeschikten, wat logischerwijs volgt uit de plichten behorende bij hun rol. Ook scoren de militaire leiders hoger op zelf-consistentie. 


\section{Emoties in morele oordeelsvorming}

Met welke verschillende typen ethische vraagstukken krijgen militairen in de dagelijkse realiteit van uitzending te maken, en hoe zijn deze gerelateerd aan ervaren emoties en de respons- strategieën: morele zelfafmelding, afstomping en relativisme?

De studie beschreven in Hoofdstuk 4 exploreerde de samenhang tussen verschillende typen ethische vraagstukken en responsstrategieën (zoals blaming the victim en morele rechtvaardiging) en hoe deze samenhang versterkt wordt door ervaren emoties. Onderzoek naar morele emoties, (Haidt, 2001), morele afstomping (Lifton, 1973), ethisch relativisme (Whetham, 2008) en morele zelf-afmelding (ook wel moral disengagement, Bandura, 1999) vormden de basis voor deze studie. Dezelfde 45 interviews als in hoofdstuk 3 werden gecodeerd en geanalyseerd. Werk gerelateerde ethische vraagstukken werden het meest beschreven, gevolgd door culturele ethische vraagstukken. Van de emoties werden anderen-veroordelende emoties (zoals woede en minachting) het meest benoemd. Relativisme werd het meest beschreven als gebruikte responsstrategie.

Morele rechtvaardiging, relativisme en afstomping toonden een sterke correlatie met culturele en werk gerelateerde ethische vraagstukken. Anderen-veroordelende emoties lieten een mediërend effect zien op de relatie tussen morele rechtvaardiging en culturele/ werk gerelateerde ethische vraagstukken, evenals op de relatie tussen culturele ethische vraagstukken en relativisme. De uitkomsten van de studie suggereren dat de context bepalend is voor de respons-strategieën en dat het belangrijk is om emoties aandacht te geven, omdat zij de reacties van militairen in ethische vraagstukken sterk inkleuren.

\section{Sensemaking tactieken in morele oordeelsvorming}

Hoe is de morele intensiteit van een situatie gerelateerd aan het gebruik van bepaalde tactieken om betekenis te geven aan een situatie gedurende militaire operaties?

In de derde en laatste empirische studie, gepresenteerd in Hoofdstuk 5 is morele oordeelsvorming vanuit de geïntegreerde benadering onderzocht. We hebben bestudeerd op welke wijze individuen betekenis geven aan ethische vraagstukken in een complexe omgeving en hoe dit samenhangt met de ervaren morele intensiteit van de situatie. Morele intensiteit (Jones, 1991) hangt af van de kenmerken van een situatie, zoals de ernst van de gevolgen, de waarschijnlijkheid van een negatief effect en de nabijheid en persoonlijke relevantie van de situatie en de betrokkenen voor de beslisser.

De person-situation interactionist approach zoals voorgesteld door Treviño (1986) gaat ervan uit dat de situatie invloed heeft op individuele morele oordeelsvorming, wat het uitgangspunt vormde van deze studie. We verkenden op welke wijze individuen een constructie maken van de situatie waarin zij zich bevinden aan de hand van een raamwerk gebaseerd op eerdere studies (Brock et al. 2008; Caughron et al., 2011; Thiel, et al., 2012). Sensemaking betreft vier categorieën van tactieken die door individuen ingezet 
worden: emotie regulatie, voorspellen, zelfreflectie en integreren van informatie (vgl. Brock et al., 2008; Thiel, Bagdasarov, Harkrider, Johnson \& Mumford, 2012). Emotie regulatie betreft tactieken gericht op het verminderen en voorkomen van emotionele overweldiging. Voorspellen betreft tactieken waarin individuen anticiperen op mogelijke gevolgen van verschillende alternatieve handelingsopties. Zelfreflectie betreft tactieken die laten zien dat men zich bewust is van persoonlijke voorkeuren (biases) en van een morele reflectie op de situatie. De laatste categorie betreft tactieken die individuen helpen om beschikbare informatie te analyseren en te integreren zoals het consulteren van anderen.

Twee prototypische voorbeelden van ethische vraagstukken (één laag en één hoog in morele intensiteit) uit de interviews werden middels een vragenlijst aan 325 actief dienende militairen voorgelegd. De resultaten lieten zien dat wanneer de morele intensiteit hoger is, minder aandacht uitgaat naar tactieken die cognitieve processen van een hogere orde vereisen, het zogenaamde kritisch nadenken, zoals zelfreflectie. Daarnaast bleek dat militairen die vaker op uitzending waren geweest een bepaalde mate van 'morele gevoelloosheid' leken te tonen doordat zij de situaties als minder intens beschreven dan hun minder ervaren collega's. De uitkomsten suggereren dat er nog ruimte is voor verdere versterking van de morele professionaliteit, juist in sterk intense situatie. Net als in hoofdstuk 3 wijzen de uitkomsten van deze studie op de mogelijkheid tot het versterken van individuele reflexiviteit en kritisch nadenken in situaties die hierom vragen.

\section{Morele uitdagingen}

Met het afronden van de empirische studies kon er een categorisatie gemaakt worden van verschillende typen morele uitdagingen in militaire operaties die ontstaan door de interactie met anderen. Drie overkoepelende typen morele uitdagingen zijn geïdentificeerd:

A) Ethische vraagstukken die voortkomen uit de interacties met individuen met een andere culturele achtergrond. Bijvoorbeeld een dilemma door een botsing tussen persoonlijke waarden en normen enerzijds en de moraal van de lokale bevolking anderzijds, zoals met betrekking tot de omgang met vrouwen of kinderen.

B) Ethische vraagstukken die werk gerelateerd zijn,

a. die voortkomen uit een botsing tussen positief nastrevenswaardige waarden in samenwerking met de eigen troepen, zoals de botsing tussen loyaliteit naar collega's en het opvolgen van regels en orders gegeven door een commandant.

b. die voortkomen uit de samenwerking met coalitiepartners die er andere werkwijzen en een andere arbeidsmoraal op nahouden 
(bijv. persoonlijke normen versus de norm van de coalitiepartner, zoals in omgang met gevangenen of het aannemen van contanten).

C) Ethische vraagstukken die voortkomen uit interacties met het thuisfront, waarin belangen botsen. Zoals de twijfel die ontstaat over welke ervaringen wel en niet te delen om het thuisfront te beschermen en tegelijkertijd het hart te kunnen luchten.

Doordat deze ethische vraagstukken, ongeacht rang, werden beschreven en in de dagelijkse uitvoering van de werkzaamheden in militaire operaties kunnen worden ervaren, is moraliteit een belangrijk thema voor alle niveaus van de militaire organisatie.

Recente militaire operaties hebben ons geleerd dat het operationele veld niet gevrijwaard is van morele dimensies (vgl. De Graaff \& Van den Berg, 2010). Door de sociale interacties die zij hebben met collega's, coalitiepartners, lokale bevolking en het thuisfront is het dan ook niet reëel om te geloven dat militairen geen of nauwelijks ethische vraagstukken tegenkomen wanneer zij ingezet worden. Deze dissertatie laat zien dat ethische vraagstukken niet exclusief zijn voorbehouden aan leiders en politici, maar vooral ook dagelijks voorkomen op de werkvloer. Het versterken van de individuele morele competentie is dan ook niet alleen gewenst, het is noodzakelijk. Deze dissertatie laat zien dat morele oordeelsvorming in de complexiteit van militaire operaties meer is dan het omzetten van de knop naar de moral high ground omdat het oordeel niet alleen afhangt van de morele ontwikkeling van het individu, maar beïnvloed wordt door psychologische processen. Dit vereist goede voorbereiding op de ethische vraagstukken die de individuele militair tegen zou kunnen komen. Het versterken van morele competentie is dan ook essentieel. Hierin is het belangrijk de militair te leren adequaat te reageren op situaties waarop hij niet is voorbereid en waarin processen als morele zelf-afmelding, emotionele gevoelloosheid of relativisme een rol kunnen spelen. Dit vereist goede begeleiding vanuit de organisatie om haar personeel te attenderen op processen die ongemerkt en langzaam hun intrede kunnen doen en waarbij emoties en intensiteit de interpretaties kunnen beïnvloeden. Ook vereist het om als organisatie te leren van ervaringen voor volgende rotaties en adequate ondersteuning te bieden bij de verwerking van deze, veelal toch intense en emotionele, ervaringen. 



\section{Dankwoord}


In 2009 , toen ik werkzaam was als leiderschapsadviseur bij het Kenniscentrum voor Leiderschap \& Ethiek van de Koninklijke Landmacht, had ik de eerste gesprekken over wetenschappelijk onderzoek naar psychologische processen in relatie tot 'het militaire leven'. Gesprekken met mijn leidinggevende Walter van Bijlevelt, Ellen Giebels van de

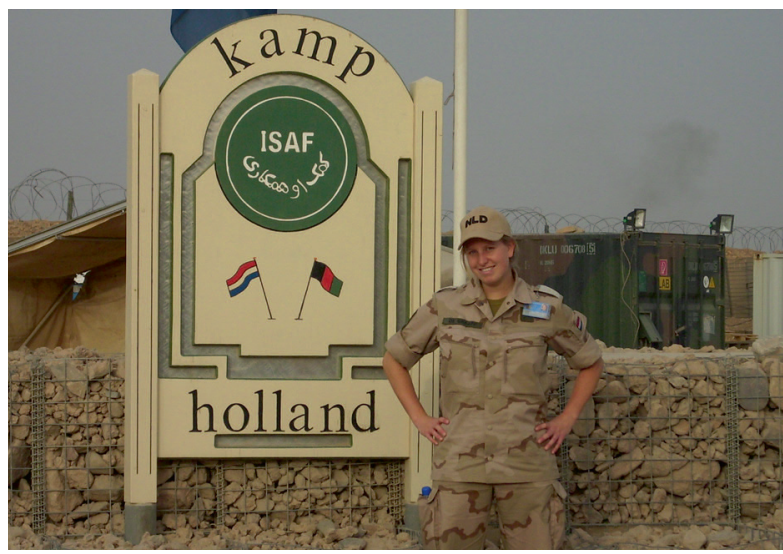
Universiteit Twente en Desiree Verweij van de Nederlandse Defensie Academie leidden er uiteindelijk toe dat ik in 2010 met de laatste rotatie van Task Force Uruzgan meeging. Enerzijds, om met mijn collega Frank Peters de militaire leidinggevenden te coachen en teams te begeleiden, anderzijds om interviews te houden voor mijn net gestarte promotieonderzoek over morele uitdagingen. Een bijzondere ervaring. Zeker ook omdat in de periode dat wij in Afghanistan waren drie Nederlandse militairen omkwamen. Door deze ervaring snap (en voel) ik de band nu beter die militairen onderling hebben na het doorleven van een uitzending. Frank, bedankt dat je met me mee bent gegaan, ook al had je gehoopt nooit meer in een ramp ceremony te hoeven staan. Ik ben blij dat jij daar met mij was.

$\mathrm{Na}$ zeven jaar literatuur doorlezen, interviews houden, observeren, vragenlijsten rondsturen, analyseren en schrijven is het nu eindelijk zo ver en mag ik promoveren. Het was niet altijd leuk en soms heb ik me ook echt afgevraagd waar ik mee bezig was, naast mijn fulltime baan bij Defensie. De inspirerende gesprekken met mijn promotoren Ellen en Desiree, de hulp van collega-PhD's en het feit dat militairen blij waren dat er aandacht was voor 'hun verhaal' hebben mij gemotiveerd door te gaan met dit onderzoek. Ellen, je stond altijd klaar - ook tijdens je vakanties en 's avonds laat - om nog even iets van me door te lezen en me feedback te geven. Dit waardeer ik zeer, hierdoor zijn de studies interessanter en beter geworden. Desiree en Ellen, dank voor jullie constante positieve houding, de mooie gesprekken die we hebben gehad en de ontdekkingen die we samen hebben gedaan in het onderzoeksmateriaal.

Promoveren doe je niet alleen. Graag wil ik de studenten bedanken die geholpen hebben en vooral Femke den Besten en Dominique Meijer, die hebben bijgedragen aan twee publicaties. Ook dank voor de andere co-auteurs: Walter van Bijlevelt, Eric Vermetten, Michelle Schut en Peter de Vries. Natuurlijk is mijn dank ook groot voor 
al die militairen die hebben deelgenomen aan de verschillende studies. Speciale dank voor het Korps Mariniers en het $42^{\text {ste }}$ Bataljon Limburgse Jagers, die deel uitmaakten van Task Force Uruzgan en het voor mij mogelijk maakten onderzoek te doen in Afghanistan. Ook wil ik mijn defensiecollega's, Maarten Rijkenbarg, Karla BoeijenMennen, Anne-Marie van Veldhuizen, Eric-Hans Kramer, Matthijs Moorkamp, Eva van Baarle, Claire Zalm, Marlies Koonen, Iris van Dijk en Yvonne Schroeder bedanken voor het meedenken, meelezen en het stellen van kritische vragen. Derek Suchard, voor jou speciale aandacht, omdat je met zoveel zorg steeds weer naar mijn teksten hebt gekeken en ze hebt verbeterd. Enorm bedankt daarvoor! Ook mijn collega's van de Universiteit Twente, vakgroep PCRS: allemaal bedankt, in het bijzonder Sven Zebel, Elze Ufkes en Margot Kuttschreuter voor de goede ideeën en hulp. En niet te vergeten alle collega-PhD-studenten: het was super leuk om met jullie in 'hetzelfde schuitje te zitten': dat we samen konden mopperen en ook elkaar konden helpen met ingewikkelde analyses. Miriam Oostinga, Wendy Schreurs, Marije Bakker en Femke Hilverda: jullie zijn top-meiden!

Mijn dank gaat ook uit naar mijn paranimfen, Marije Bakker, Michelle Schut en Vera van der Zee, die de afgelopen periode veel voor mij hebben betekend. Ook wil ik de leden van de promotiecommissie bedanken. Jullie en jullie werk hebben mij geïnspireerd. Ik vind het een eer dat jullie in mijn commissie wilden plaatsnemen.

Lieve (schoon)familie, bedankt voor jullie constante interesse in mijn onderzoek. Door lieve kaartjes en brieven met daarin jullie gedachten over mijn onderzoek, zijn jullie onderdeel van mijn dissertatie. Jullie zijn erg belangrijk voor mij en ik ben blij dat ik dit samen met jullie mag beleven. Helaas zijn mijn opa's en oma's er niet meer om dit mee te maken. Ze hadden dit prachtig gevonden. Anke en Sandra: op onze eigen unieke manier dragen we elk bij aan een stukje meer menswaardige wereld $;$-) Laurien \& Martijn, Chantal \& Mike, Sylvia \& Daniël, Andrea \& Geert, Marjolein \& Pieter, Mijke $\&$ Paul, en Ria: hoe bijzonder is het dat we al zo lang vrienden zijn! Vera \& Fokko, Pieter \& Linda, Wilbert \& Frederique, Ralf \& Nina, Martijn \& Frederique, Tim \& Jolanda: we maken er steeds weer een mooi feestje van! Annelies, Wendy, Annabelle, Sanne en Emilie: mooi dat we, na onze studietijd, elkaar nog niet uit het oog verloren zijn!

Lieve papa en mama, jullie hebben mij geleerd nieuwsgierig te zijn, door te zetten en een liefde voor mens, wetenschap en filosofie bijgebracht. Met nu dit boekje als resultaat. Dank voor jullie onvoorwaardelijke liefde en steun. 
Lieve Boy, het idee om te gaan promoveren was net in mijn hoofd geplant toen wij een relatie kregen. Je hebt veel avonden, weekenden en vakanties steeds weer die laptop uit mijn tas zien komen om toch nog even wat aan mijn onderzoek te doen. Nooit heb je hierover geklaagd (wel moet ik je vanaf nu eerst toestemming vragen voor nieuwe plannen (:). Je denkt altijd met me mee, leest stukken door en hebt me zelfs aan respondenten geholpen. Dat kunnen niet veel promovendi zeggen. Dank je voor alles, liefje! 

Curriculum Vitae \& List of publications 
Miriam Carla de Graaff (Hengelo, 1985) obtained master's degrees in both Communication Studies (Cum Laude) and Psychology from the University of Twente (The Netherlands) in 2008. Since 2008, she has been working for the Netherlands Ministry of Defense in various (managerial) functions in the field of leadership, ethics, and professional development. Miriam started her $\mathrm{PhD}$ project in 2010 under supervision of prof.

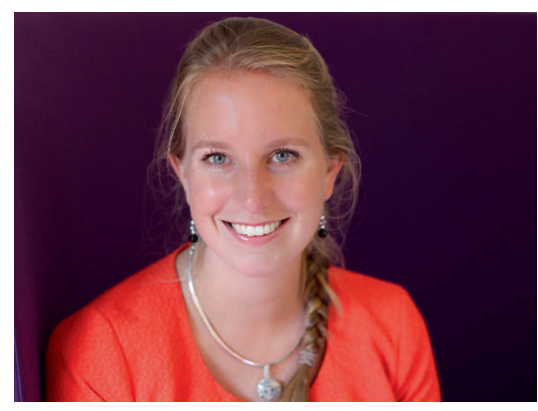
dr. Ellen Giebels and prof. dr. Desiree Verweij. Her doctoral dissertation in psychology was focused on studying the moral assessment of ethical challenges in the complexity of military operations. Miriam has presented her work at various national and international conferences, including the annual global conference of the International Leadership Association in Boston, the annual European conference of the International Society for Military Ethics in Belgrade and the bi-annual conference of the European Association of Work and Organizational Psychology in Santiago de Compostella. Miriam has published several articles regarding leadership, management and/or ethics in national and international journals and books. In 2015, Miriam was nominated for the Netherlands Young Talent Award for women in leadership.

\section{International Publications}

De Graaff, M.C., Boeijen-Mennen, K.H.M. \& Schroeder, Y.C.J., (2013). The Dutch approach: Leadership lessons from multidisciplinary teams. In D. Woychesin \& M.C. de Graaff (Eds.), The Comprehensive Approach: International Perspectives (pp. 101-114). Kingston: Canadian Defence Academy Press.

De Graaff, M. C., Den Besten, F.D.A., Giebels E. \& Verweij, D.E.M. (2016). Moral judgement in war and peacekeeping operations: An empirical review. In T.R. Elssner \& R. Janke (Eds.), Didactics of Military Ethics (pp. 75-111). Leiden: Brill.

De Graaff, M.C., De Vries, P.W., Van Bijlevelt, W.J. \& Giebels, E. (forthcoming). Ethical leadership in the military, the gap between theory and practice in ethics education. In P.J. Olsthoorn (Ed.), Ethics in Military Leadership. Leiden: Brill.

De Graaff, M.C., Meijer, D., Verweij, D.E.M., \& Giebels, E. (forthcoming). Sensemaking in Military Critical Incidents: the impact of moral intensity. Business and Society.

De Graaff, M.C., Schut, M., Verweij, D.E.M., Vermetten, E., \& Giebels, E. (2016). Emotional reactions and moral judgment: The effects of morally challenging interactions in military operations. Ethics \& Behavior, 26(1), 14-31.

De Graaff, M.C., \& Van den Berg, C.E.M. (2010). Moral Professionalism within the Royal Netherlands Armed Forces. In J. Stouffer \& S. Seiler (Eds.), Military Ethics: International Perspectives (pp. 1-24). Kingston: Canadian Defence Academy Press. 
De Graaff, M.C., \& Van Gils, M.J. (2012). Military professionalism, an organizational challenge by itself. In J. Stouffer \& D. Lindsay (Eds.), Threats to Military Professionalism: International Perspectives (pp. 57-70). Kingston: Canadian Defence Academy Press.

Kramer, E.H., De Waard, E., \& De Graaff, M.C. (2012). Task Force Uruzgan and experimentation with organization design. In R. Beeres, J. Van der Meulen, J. Soeters \& A. Vogelaar (Eds.), Mission Uruzgan, Collaborating in Multiple Coalitions for Afghanistan (pp. 235-250). Amsterdam: Pallas Publications.

Pfister, A., De Graaff, M.C., \& Van Gils, M.J. (2012). The dynamic-five-factor model as a compass for military professionalism: a comparison between Switzerland and the Netherlands. In J. Stouffer \& D. Lindsay (Eds.), Threats to Military Professionalism: International Perspectives (pp. 195-216). Kingston: Canadian Defence Academy Press.

Schut, M., de Graaff, M. C., \& Verweij, D. (2015). Moral emotions during military deployments of Dutch forces. A qualitative study on moral emotions in intercultural interactions. Armed Forces \& Society, 41(4), 616-638.

Woychesin, D. \& De Graaff, M.C. (2013). The Comprehensive Approach, international perspectives. Kingston: Canadian Defence Academy Press.

\section{Publications in Dutch}

De Graaff, M.C. \& Kramer, E.H. (2009). Onderling vertrouwen binnen uitgezonden eenheden: een kwalitatief onderzoek. Militaire Spectator, (178) 4, 221-234.

De Graaff, M. C., \& Kramer, E.H. (2012). Leiderschap, uitzending en 'intelligent failure'. De intelligente mislukking als hoeksteen van de lerende organisatie. Management en Organisatie, 66(5), 41-60.

De Graaff, M.C., Schut, M., Zalm, C.E. (2016). Morele fitheid in militaire inzet, de noodzaak van aandacht voor integriteit ook in het 'echte' werk. In N. Boonstra et al. (Eds), Jaarboek Compliance 2017 (pp. 47-64). Capelle aan den IJssel: Nederlands Compliance Instituut.

De Graaff, M.C. \& Stoppelenburg, D. (2013). Destructief leiderschapsgedrag, Carré, (5), 28-33.

De Graaff, M.C. \& Stoppelenburg, D. (2013). Onbewust ongewenst leiderschapsgedrag. De Onderofficier, 55(4), 14-19. 


\section{MORAL FORCES}

\section{Interpreting Ethical Challenges in Military Operations}

The studies presented in this dissertation reveal three broad types of ethical challenges during military operations at an individual level that are caused by social interactions of military personnel, regardless of rank. The first encompasses ethical challenges related to encounters with other individuals from another cultural background, like the local population. The second comprises work-related interactions such as those with fellow team members. The third are ethical challenges as a result of interactions with the home front. This dissertation addresses moral assessment: the processes involved with how individuals interpret ethical challenges and how they respond to them. Psychological mechanisms influence this moral assessment and are the main focus of this dissertation. The empirical studies address, in order of presentation in this dissertation: moral identity, moral disengagement, emotions, numbing, relativism, sensemaking, and moral intensity.

ISBN: 978-90-365-4279-1

DOI: $10.3990 / 1.9789036542791$ 